0000111011100000 1

\title{
BANAFSHEH HAJINASAB
}

A DYNAMIC APPROACH

TO MULTI-AGENT-BASED

SIMULATION IN URBAN

TRANSPORTATION

PLANNING

W. (1)

$-\frac{1}{4}$

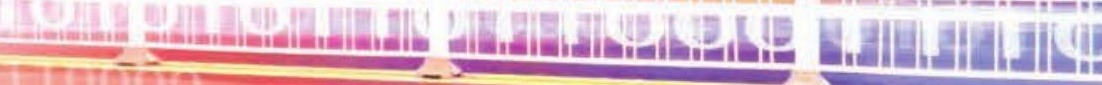

Tor:

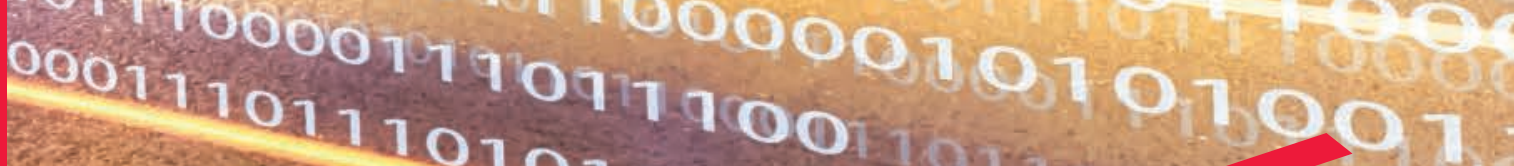



A DYNAMIC APPROACH TO MULTI-AGENT-BASED SIMULATION IN URBAN TRANSPORTATION PLANNING 
Studies in Computer Science no 5

(C) Copyright Banafsheh Hajinasab, 2018

ISBN 978-9I-7I04-924-7 (print)

ISSN 978-9I-7I04-925-4 (pdf)

Holmbergs, Malmö 2018 


\section{BANAFSHEH HAJINASAB}

A DYNAMIC APPROACH TO MULTI-AGENT-BASED SIMULATION IN URBAN TRANSPORTATION PLANNING

Malmö University, 2018 Technology and Society 


\section{Earlier publications in this series}

Jevinger, Åse. Toward intelligent goods: characteristics, architectures and applications, 2014, Doctoral dissertation.

Dahlskog, Steve. Patterns and procedural content generation in digital games: automatic level generation for digital games using game design patterns, 2016, Doctoral dissertation.

Fabijan, Aleksander. Developing the right features: the role and impact of customer and product data in software product development, 2016, Licentiate thesis

Paraschakis, Dimitris. Algorithmic and ethical aspects of recommender systems in e-commerce, 2018, Licentiate thesis

The publication is also electronically available at: www.mah.se/muep 
To my lovely caring daughter, Sheida 



\section{ABSTRACT}

Reviewing previous studies on using computational models for analyzing the effect of transport policies on transportation systems shows that agent-based models have not been used much in spite of their great potential for simulating dynamic aspects of policy instruments and travel behavior. The main reason can be the need for a lot of input data which is hard to prepare for the modeler. This has led to limited use of agentbased models in previous studies and even in those studies the scope of simulation is limited to only particular scenarios. In this thesis, I proposed a general-purpose agent-based simulation model for urban transportation that supports simulation of a wide range of policy instruments. The proposed model is designed in a way that a large part of the input data can be generated automatically using online web-services. The thesis also reports an empirical study on using our proposed generalpurpose model together with on-line travel planners in agent-based simulation for predicting the effect of different policy instruments on travel behavior. The results from our empirical study showed that our generalpurpose agent-based model predicts $72 \%$ of the real travel decisions correctly. Furthermore, the results of the simulation for various scenarios and combination of them seem to be acceptable. Finally, we found out that the use of on-line services for data collection increases the speed and flexibility of the system for defining and running new scenarios. However, the scalability of using on-line services in simulation is constrained by limitations of online service providers.

The main contributions of this thesis are a general-purpose agentbased simulation model for urban transportation and a novel approach to automatically generate input data to the simulation using online travel planners and other web-services. This novel approach mitigates the challenge of agent-based simulation as a data-intensive method. This can lead to more widespread use for agent-based simulation in solving complex and realistic transportation scenarios.

Another contribution of this thesis is on visualization of simulation output. One of the main challenges of using simulation systems by transport planners and decision makers as end-users is to understand the com- 
plex output of the simulation. In this thesis, I empirically demonstrated how the usability of a freight transport simulation system is improved by adding a visualization module that illustrates the results of the simulation for the end-users. 


\section{ACKNOWLEDGMENTS}

I would like to express my special appreciation and thanks to my supervisors Professor Paul Davidsson, and Docent Jan Persson. I would also wish to express my gratitude for the help I got from Dr. Johan Holmgren and Professor Jonas Löwgren along the way.

I am also thankful for the support I received from my colleagues at Malmö University, Trivector Traffic, and European Spallation Source. Special thanks to Emeli Adell, Karin Rathsman, Susanne Regnell, Zahra Hamidi, Annabella Loconsole, Amina Agovic, Nathalie Schuterman and many others that supported me during my $\mathrm{PhD}$ time in so many ways.

To my friends outside work, I am so grateful to get to know you all: special appreciation goes to Niloufar, Azadeh, Saghar, Nastaran, and Shohreh. Thanks for all the great times together!

Finally, but by no means least, thanks go to my parents, my amazing daughter Sheida, and Shahram for their support.

This research project was supported by K2 - The Swedish Knowledge Centre for Public Transport, Malmö University, and the National ITS Postgraduate School. 



\section{PREFACE}

The dissertation is organized into two main parts. Part I provides an overview of the research background, research method, and the main findings. Part II consists of a collection of 6 papers that describe simulation models, system implementation, and evaluation of the systems that are summarized and discussed in Part I. To maintain the consistency, readability and presentation of the original publications, the papers are included in their publicly available published formats.

\section{Part I consists of 5 chapters:}

Chapter 1 Introduction

The introduction chapter, a broad background and research context is given about advantages and limitations of agentbased simulation in comparison with traditional approaches for investigating the effect of policy instruments on travel behavior.

Chapter 2 Research focus

This chapter explains the problem statement and the gap in the previous research that motivates our research questions. The research questions are formulated in this chapter.

Chapter 3 Research method

This chapter explains the research method taken to answer our research questions.

Chapter 4 Contributions

An overview of the main contributions of this thesis is explained in this chapter.

Chapter 5 Conclusions

This chapter concludes my findings in this thesis and proposes some future research directions. 


\section{Part II consists of 6 papers:}

Paper 1 Hajinasab, B., Davidsson, P., Persson, J. A. (2014). A survey on the use of computational models for ex ante analysis of urban transport policy instruments. Procedia Computer Science, 32, 348-355. (8 pages)

This paper provides an analysis of the use of computational models for predicting the effects of different policy instruments on urban transport systems.

Paper 2 Hajinasab, B., Davidsson, P., Persson, J. A., Holmgren, J. (2016) Towards an Agent-based Model of Passenger Transportation. Multi Agent Based Simulation XVI, Lecture Notes in Computer Science, 9568, 132-145, Springer. (14 pages)

In this paper, we present an agent-based simulation model for supporting the decision making in urban transport planning. The model can be used to investigate how different transport infrastructure investments and policy instruments will affect the travel choices of passengers.

Paper 3 Davidsson, P., Hajinasab, B., Holmgren, J., Jevinger, A., Persson, J. A. (2016). The Fourth Wave of Digitalization and Public Transport: Opportunities and Challenges. Sustainability, $8(12), 1248$. (16 pages)

In this paper, we investigate the opportunities and challenges of the forth wave of digitalization, also referred to as the Internet of Things (IoT), with respect to public transport and how it can support sustainable development of society.

Paper 4 Hajinasab, B., Davidsson, P., Holmgren, J., Persson, J. A. (2017). On the use of on-line services in transport simulation. Transportation Research Procedia, 21, 208-215. (8 pages)

In this paper, we introduce a new approach for collecting data for transport simulation models that is using on-line services in order to outsource parts of the modeling and computation of simulation models.

Paper 5 Hajinasab, B. Davidsson, P., Persson, J. A. (2017). An on-line agent-based approach to estimate the effects of transport policy instruments: An empirical study of commuting in urban areas, Submitted for journal publication (18 pages)

This paper reports an empirical study on using on-line travel planners in agent-based simulation for predicting effect of different policy instruments on travel behavior. First, we investigated the importance of different policy instruments in southern Sweden through a survey among transport decision 
makers. Then we developed an agent-based simulation system to predict the effect of the most important policies on travel behavior.

Paper 6 Hajinasab, B. Davidsson, P., Löwgren, J., Persson, J. A. (2017). Visualization of data from transportation simulation systems, Proceedings of 13th WCTR Conference, 2013, Rio de Janeiro, Brazil. (18 pages)

In this paper we investigate how visualization techniques could address the challenges of transportation simulation data analysis in order to facilitate the decision-making process. 


\section{CONTENTS}

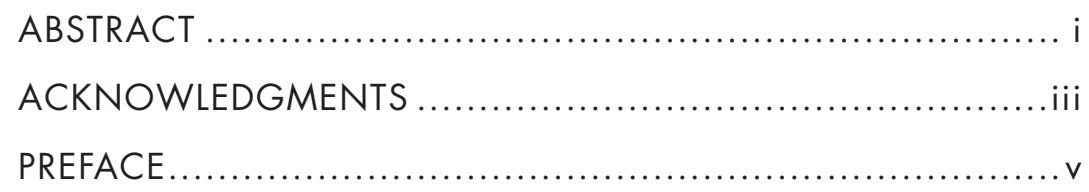

\section{INTRODUCTION ............................................ I}

1 BACKGROUND ............................................... 3

1.1 Policy instruments in transport planning ......................... 4

1.2 Model versus simulation ......................................... 4

1.3 Use of computational models in analyzing the effect of policy instruments ...................................................5

1.3.1 Top-down approaches ................................... 5

1.3.2 Bottom-up approach .....................................6 6

1.3.3 Simulation ............................................... 6

1.4 Agent-based models in transport simulation ..................... 7

1.4.1 Advantages of agent-based models.......................7

1.4.2 Drawbacks of agent-based models ....................... 8

1.5 Modeling travel behavior in uncertainty ....................... 9

1.6 Effects of ICT on travel behavior .............................. 10

1.7 Summary ...................................................... 11

2 RESEARCH FOCUS ....................................... 13

3 RESEARCH METHOD ....................................... 17 
4.1 A survey on using computational models for evaluating effect of policy instruments

4.2 Using web services as efficient sources of input to agentbased simulations

4.3 ASIMUT: a general-purpose model for simulating

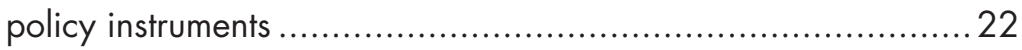

4.3.1 A utility function for modeling travel decision.............23

4.3.2 ASIMUT for simulating policy instruments ................. 25

4.4 A step-by-step approach for using ASIMUT ...................... 27

4.4.1 Step 1: Requirement analysis................................ 27

4.4.2 Step 2: Model adoption........................................ 27

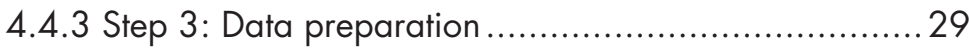

4.4.4 Step 4: Model calibration ....................................... 30

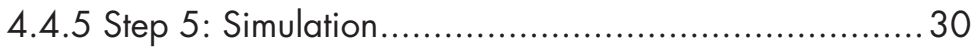

4.5 A visualization tool for transportation simulation................. 31

5 CONCLUSIONS ............................................ 33

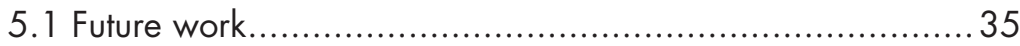

II PAPERS ................................................ 43

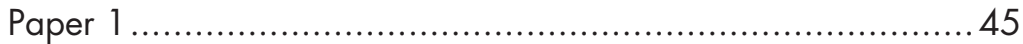

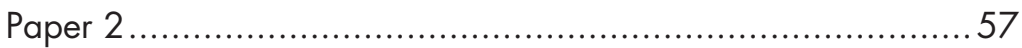

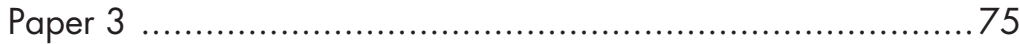

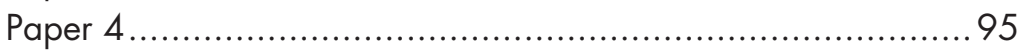

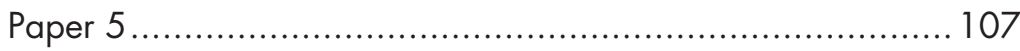

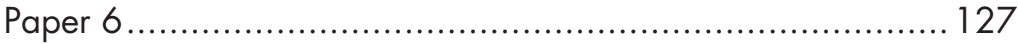




\section{INTRODUCTION}





\section{BACKGROUND}

The demand for mobility and transport as one of the crucial human requirements is constantly increasing. Nowadays with the development of transportation systems, passengers have the possibility to travel a longer distance for work and study purposes, and in general people's accessibility to various places and services has increased. Passenger transport has also some negative consequences such as emissions and traffic congestion, particularly in urban areas. Therefore, it is vital to design suitable transport strategies that encourages modal shift to public transport or non-motorized modes of transport. Designing effective transport strategies is a very challenging task. There is a wide set of policy instruments that can be used as an aid for designing transport strategies in order to develop passenger transport while reducing the aforementioned negative consequences. The policy instruments include both transportation policies and investments in infrastructure. Some examples of transportation policies are changing fuel prices, road taxes, public transportation fares, etc. while e.g., building a new train station or road is a change in the infrastructure. Changes in transportation policies and infrastructure investments may have substantial consequences on the travelers? behavior. Thus, it is very important to assess the impacts of such changes before implementation. One way of doing this is to perform experimental studies in the real world, but such studies are often very expensive and time-consuming, and sometimes unfeasible. Several studies have used variations of computational models in order to estimate the effects of policy instruments.

This chapter aims at providing an overview of the research context by reviewing the concept of policy instrument, the use of computational models in transportation planning, comparing the advantages and drawbacks of traditional top-down approaches with more recent bottom-up approaches (agent-based models) in modeling the effects of transport policies, and the challenges of travel behavior modeling. Agent-Based Model (ABM) is defined as a computational model in which the system is modeled as a collection of autonomous decision-making entities called as agents that interact with each other and their environment. 


\subsection{Policy instruments in transport planning}

Transport policy making starts with definition of a high-level goal describing the aims that the transport planners ultimately want to achieve. Examples of such objectives are reduction of congestion, private vehicle use, emissions, or more generally, travel demand management. At the next level, the transport planers establish specific and achievable objectives. Some example objectives that contribute to the mentioned sample goal can be: to reduce traffic congestion delay or to reduce barriers to non-motorized travel. The last stage includes the selection of policy instruments in order to achieve the objectives [35].

There is a wide set of policy instruments that can be used for designing transport strategies. Policy instruments range from more conventional instruments such as land use regulation, vehicle regulation, infrastructure investment, and pricing schemes, to newer instruments such as application of information technology to improve resource allocation and service quality, as well as attitudinal changes [3]. The policy instruments are very different in nature and have different effects on different transport systems. Moreover, the combination of policy instruments might have a totally different effect on the transport system compared to applying each individual instrument alone [36]. It has been argued that the development of sustainable transport strategies often fails due to lack of integration of different policy instruments [43, 44].

Before we discuss the use of of computational models in analyzing policy instruments, we need to clarify the terminology used in this thesis. For example, what we mean by model, computational model, simulation model, and simulation tool.

\subsection{Model versus simulation}

A model is a purposeful representation of a real world system [46]. The modeler makes this purposeful representation to solve a problem or answer some questions. In the modeling cycle, the modeler first builds a conceptual (non-computational) model of the real system and after validating the conceptual model, a computational model is generated based on the conceptual model [4]. The computational model is a mathematical representation of the real system that can be used in a simulation. In this thesis, whenever we use the term model we mean computational model. Since the focus of this thesis is transportation simulation, we use the term simulation model as a particular type of computational model that is used in a simulation. Finally, by simulation tool we mean the software that is developed to run the simulation based on a simulation model. 


\subsection{Use of computational models in analyzing the effect of policy instruments}

In order to know which policy instruments should be chosen in a particular situation, it is important not only to have knowledge about availability of policy instruments, but also their potential impact on the transport system. There are different methods to analyze the effects of policy instruments. Kremers et al. [34] have categorized methods for transport policy analysis into two main types: 1) qualitative ad-hoc approaches that are solely based on expert judgment or interviews and 2) quantitative structured approaches, where a statistical or econometric model based on quantified data is used. Ad-hoc approaches are typically used in situations where there is no possibility for a structured approach due to the time constraints, non-repetitive situations, or lack of data. The computational models used in the previous work falls into two main categories: 1) top-down (traditional) approaches and 2) bottom-up approaches.

\subsubsection{Top-down approaches}

The predominant type of passenger transport analysis models, which are used by public authorities on regional, national, and international levels in order to support their decision-making, is the so-called macrolevel models. Macro-level models are based on highly aggregated data, and they are often described as top-down models as they are built with the purpose to reproduce known (aggregated) transport statistics, typically on a national level. Examples of such models are the Swedish national passenger transport modeling system (Sampers) [1], and TRANSTOOLS [15], which is an EU-level model for passenger and freight transport modeling [15, 42]. Macro-level models are in general steady-state models, where time is not explicitly modeled, even though they might include components that are based on dynamic modeling. This means that, for example, bus and train departures in macro-level models are typically modeled using frequencies and average travel times instead of using timetables. As macro-level models use aggregate data, it can be argued that the amount of data that needs to be collected, and included in the model, can be considered to be rather reasonable; at least when comparing with micro-level modes, which we discuss below. Moreover, in macro-level models, the data is in general included as a part of the model. 


\subsubsection{Bottom-up approach}

Micro-level modeling is a bottom-up approach since it describes individuals in population with micro-level data e.g., census data and simulates the individuals' behavior based on the population statistics. In microlevel (bottom-up) modeling the population is generated by aggregating the simulated individuals' data. Agent-based models are a special type of micro-level models, but their focus is more on the interactions among individuals. One property of ABMs is their ability to capture unexpected or emergent behavior generated by these interactions. Agent-based and micro-level models are often referred to as bottom-up models since they both take the bottom-up approach to replicate the population dynamics, and each individual has its own properties. However, in micro-level models the interaction between individuals are often missing while in ABM agents' behaviors influences on other behaviors. [5]

\subsubsection{Simulation}

Simulation method has been used in many areas of research including social science in order to experiment situations which would have been difficult to examine in real world. Simulation is an experimental method in which the researchers create and use a representation of a real world or hypothetical phenomenon in order to answer to some questions about that phenomenon. The simplified representation of the real world phenomenon is called a model and the real world phenomenon itself is called a target. Performing experiments with a model of target is simpler, faster and more feasible than experimenting the target itself $[24,40]$. The simulation method is very suitable for the target of urban transportation where the target of the simulation, i.e. travel behavior is very difficult to do experiment on in real world. The logic of simulation is shown in Fig. 1.1. In the area of urban transportation modeling, the target relates to the travel behavior of the urban passengers. The modeler collects data about the target and other relevant information such as origin-destination matrixes and transport systems, etc. At the next step, he modeler creates an abstraction model of the target. The abstraction model represents the real world in a simplified way by filtering out some aspects of the system that is not significantly relevant for the purpose of study. Thus, the purpose of the model, i.e. the questions that the modeler wishes to answer with model, is very determining in the process of building and simplifying the model. Running the model, the simulation generates some simulated data which later the modeler will compare to the real world collected data of the target in order to check the similarity of the modeled target with the target itself. 


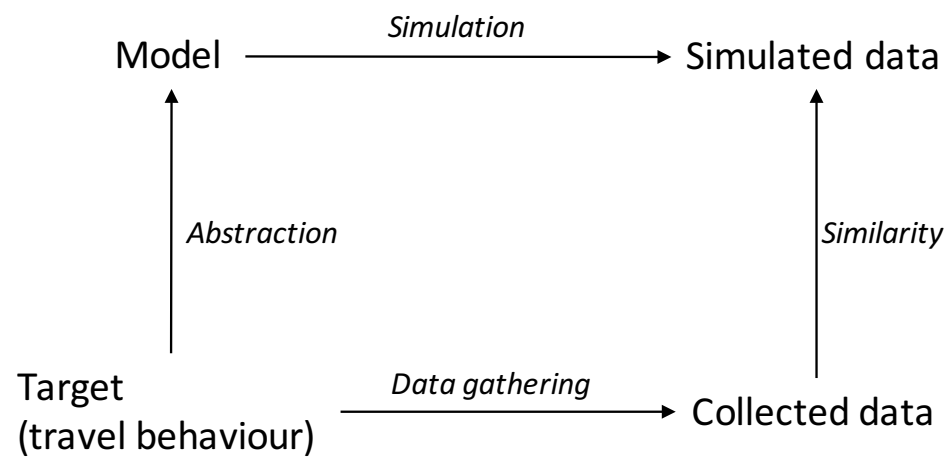

Figure 1.1: The logic of simulation method in transport research [24]

\subsection{Agent-based models in transport simulation}

In agent-based modeling $(\mathrm{ABM})$ instead of describing the system through the variables representing the state of the whole system, its individual entities are modeled. An agent-based model consists of a system of agents and the relationships between them. Each agent individually assesses its situation and makes decisions. Agents act according to their own individual goals, pointing to the autonomous aspect of agents. The population of agents is usually heterogeneous, meaning that each individual is different and has different characteristics $[8,12,40]$.

In the ecology discipline, the term Individual-Based Model (IBM) is used more often than ABM while they both refer to the same concept. However, there have been some historical differences between IBM and ABM: the focus of IBMs has been individual variability and local interactions while the main focus of ABMs has been decision making and adaptive behavior. [40]

The agent-based modeling paradigm has become a popular tool for modeling and understanding many complex systems. This approach has also gain attention in transportation science, and it can be a very useful approach specially for modeling travel behavior of individuals e.g., [9, 20].

\subsubsection{Advantages of agent-based models}

Agent-based modeling is a more dynamic approach compared to traditional top-down approaches with respect to the level of detail in modeling different parts. For instance, more interesting parts of the infrastructure can be modeled with a higher granularity. This makes it possible to study the effects of, e.g., building a new bike parking facility that is 
safe and efficient and close to a train station, or allowing the travelers to bring their bikes on the trains. Furthermore, the agent-based approach is structurally capable of modeling what travel options different travelers actually are aware of, or consider, when deciding what option to choose. This makes it possible to study the effects of, e.g., travel awareness campaigns and the availability of advanced travel planning systems. These interventions are difficult, or even impossible, to study using traditional models.

Furthermore, agent-based models are able to capture time-related aspects, such as the effects of synchronization and optimization of timetables [41]. There are many transport policy measures that concern time, e.g., time-differentiated congestion and parking fees. Such transport policies are difficult to study using traditional top-down models, but they may have an important influence on travel choices. The way that agent-based models handle the time falls into two main categories: 1) simulation of day-to-day planning where learning from the past experience of a single traveler is included in the model; 2) within-day in which the behavior of traveler agent is modeled within a day without considering the effect of past experience of the agent. In this thesis, we took a within-day approach since the data we used for calibration was limited to travel diaries of a person for only one day.

Using an agent-based approach for modeling travelers, including their behavior and interaction with each other and the surrounding environment, will facilitate capturing each individual's preferences and characteristics. This is critically important in order to determine the actual decisions of individual travelers. Thus, agent-based modeling seems very well suited to predict and analyze the effects of different transport measures, since it explicitly models the decisions of each individual and is able to compute the consequences of these decisions.

\subsubsection{Drawbacks of agent-based models}

From a data perspective, agent-based models (or micro-level models in general) are more data intensive, as they require data describing all the modeled entities (e.g., $[2,17,25,37,50])$. Obviously, it is possible to use statistical distributions in order to model the diverse behavior of the individuals, but such an approach still requires quite some effort on collecting micro-level data that represents the modeled population. As agent-based models are dynamic in nature, they also need data that allows to model entities over time, such as timetables for buses and trains. Obviously, this means that the amount of data that needs to be collected and included in the models can be quite large, in particular when studying large scenarios. Therefore, it is not always possible to build agentbased models where all of the required data is included as a part of the model, which is the case for the macro-level models discussed above. 
Agent-based models are therefore sometimes used as modeling frameworks, including blocks to build different analysis models. An example of such frameworks is the MATSim modeling framework [6,32], that can generate different types of passenger and freight transport models. Through the use of modeling frameworks, it is possible to develop models with a minimum amount of data, as it is possible to tailor the data collection to the specific needs of a model. In general, data can be collected for only the modeled entities and the part of the network that are modeled. However, as the number of entities and the size of the studied geographic region grows, it becomes more and more demanding to collect the data, regardless whether or not a framework is used.

\subsection{Modeling travel behavior in uncertainty}

Understanding the incentives and factors that affect the passengers' behavior in an unpredictable world is one of the most complex issues in transport policy planning [13]. The most dominant paradigm for modeling travel behavior in transportation research is rational choice where a traveler chooses an alternative which maximizes her utility. Utility is calculated based on the attributes of a travel alternative, for instance travel cost and time, comfort, environmental friendliness, etc. A discrete choice analysis approach can be used to model travel behavior, for instance, for modeling mode and route choices [10]. In addition due to simplicity and mathematical elegance of the utility-based models, these models handle many unknown factors affecting individual choice by their flexible structures of error [39]. However, there is always a main critique for rational choice approach that is to be normative which means the model explains how rational individuals would decide, not how actual travelers decide. The real decision making is affected by many factors such as different types of uncertainty, emotions, lack of having all needed information, different perception of risks for each travel alternative, etc. [13]. To overcome this problem several approaches have been proposed by the transportation research community for providing a more realistic model of human decision making. Some examples of decision making approaches explaining human behavior in uncertainty are explained briefly as follows.

1. Adopting context-dependent heuristics [7]: This approach focuses on limitation of human cognition. In this approach, humans are assumed to be rationally bounded which means they cannot acquire and process all information about all possible alternatives [23]. Instead they focus on the most important attributes and apply simple heuristics to take decision [45].

2. Prospect Theory [49]: Prospect Theory allows for a subjective 
weighting of perceived probabilities reflecting the decision maker's attitude to risk. According to Prospect Theory, decisions are contextdependent and evaluation of risky prospects includes assessment of behavioral outcomes with regard to some common reference points. The reference points change from one context to the other and over time which means that information provided to the travelers in different steps e.g., pre-travel or en route can have different effects on their behavior.

3. Law of effect [16]: People usually recall extraordinary and particularly bad events and forget unremarkable or normal occasions. The travelers are more likely to recall the experience of missing a train or being late for the meeting than the ones when everything worked according to the plan. This means that travelers rely on their past experience to from a knowledge on the distribution of outcome that can be far from a rational choice.

Since the focus of this thesis is to explore the possibility of using web services to provide input data to the transportation simulation models, we have not modeled the uncertainty in the transportation model. Another reason why we took a deterministic approach and did not include uncertainty was the limited empirical data available for calibrating the system. As a future study, the proposed model in this thesis can be extended to include uncertainty by adding probabilty distributions for decisions taken by agents.

\subsection{Effects of ICT on travel behavior}

Before widespread use of personal computers which was called as the first wave of digitization, travel information was limited to a combination of formal information provided by transport operators and word of mouth e.g. seeking advice from friends, relatives, etc. This has changed after emerging the Internet in 1990s that was referred as second wave of digitization. Through the early travel planners (e.g., OV9292 in the Netherlands or Transport Direct in the UK), travel information was not only available for those who were physically present at the source of information. Connected user were also able to access to the real-time travel information remotely. The third wave was advancing mobile and wireless communication technologies that allowed connected travelers to access real-time travel information anytime anywhere while they are stationary or on the move. [48]

Nowadays with the fourth wave of digitization which is called Internet of Things (IoT), it is not just people who use the Internet to access and share information, but also different types of entities, such as vehicles, appliances, and machinery. This allows to collect travel data from 
not only travelers but also transportation infrastructures and vehicles. The data can be either provided automatically through different types of sensors and machines, or via the travelers themselves through different types of smart-phone applications. Such applications could either collect data automatically (e.g. position and velocity) or manually (e.g. traveler's preferences and their final destinations). As the data typically is collected in real-time, it has the potential to impact real-time decisions by transport operators and travelers.

Previous studies showed that providing travel information to the travelers help with speeding up learning travel time distribution under unfamiliar conditions [19]. Moreover, travel information reduces stress and increases a sense of self-control in travelers [33]. Travel information is also useful from a network point of view since it helps travelers to avoid disruptions and delays caused by accidents or bad weather [33]. Previous research has also shown that travelers have an increasing tendency to rely on prescriptive information (e.g. provided by travel planners) and not decide in the dark [11]. It can be predicted that travelers' behavior will increasingly be changing towards what online travel planners prescribe. This opens new opportunities to use the output of online travel planners for modeling travel behavior [26]. Apart from the important effect of online travel planners on travel behavior, using output of online travel planners can automatize part of the data preparation for travel behavior simulation. As we mentioned earlier in this chapter, agent-based simulation is a data-intensive process that requires a huge amount of input ranged from transportation network model to the socio-demographic attributes of travelers. These data are usually collected through cumbersome and time-consuming procedures such as surveys, analyzing historical data, and calculating origin-destination matrices. Using online travel planners to provide the input data for agentbased simulation models tackle one of the main obstacles against using agent-based simulation in serious scenarios. In the following chapters of this thesis, we describe how using online travel planners and other online web-services can facilitate data preparation for an agent-based transport simulation to analyze the effect of policy instrument on travel behavior. This approach of using automated data imputation can also be applied to any other travel behavior model as a new generation of accessing up-to-dated and detailed network data.

\subsection{Summary}

A literature review on the use of computational models in transportation planning [27] showed that despite the great potential of agent-based approach in modeling the effect of transport measures on travel behavior, only few studies have applied an agent-based modeling approach in the 
context of transport policy analysis, and in most cases, the agent-based models have been very simple and do not realize the potential of the approach $[2,37]$. These models are mostly developed to investigate the effects of a specific transport measure concerning a specific scenario. Furthermore, they do not include all modes of transportation. The input variables, the model construction, and the collected output are very much chosen with a specific scenario in mind. Therefore, existing agentbased models could not be used to investigate the effects of various kinds of transport measures in different scenario settings, and there is a need for a general-purpose agent-based model for investigating the effect of various policy instruments; however, the general-purpose agent-based model needs to be designed in a way that does not require intense data preparation. 


\section{RESEARCH FOCUS}

Most of the existing work on using computational models for investigating effect of policy instruments only take into account a limited number of the factors influencing travel behavior such as cost, time, convenience, weather, etc. [27]. Moreover, most of the previous studies have applied some kind of statistical model for prediction of travel behavior change. These models have been criticized for neglecting the interaction effects between travelers and oversimplification which can introduce significant biases in output. On the contrary, agent-based models are dynamic in nature that allow to model entities over time [41]. Agent-based models allow for modeling each individual as an agent who takes travel decisions dynamically over time [24, 47]. In fact, time is an important aspect in studying many policy instruments such as time-differentiated congestion and parking fees, and it is not easy to use traditional models for investigating the effect of such policies on travel behavior. In this thesis we have not modeled congestion explicitly because traffic simulation is out of the scope of this thesis. To analyze congestion, a simulation system needs to model travelers and also the physical aspects of vehicles. The focus of this research was to investigate the effect of policy instruments on traveller's behavior not traffic system and vehicles. In long term, the effect of congestion should appear in the time prediction made by online travel planners which is used as an input to our model. Moreover, the output of our model can be used to study the congestion in a future study.

However, despite the great potential of agent-based approach in modeling the effect of transport measures on travel behavior, there are few studies that have applied an agent-based modeling approach in the context of transport policy analysis [27]. Most of these models [2,37] only demonstrate some potentials of the agent-based approach. These models are mainly proposed to study a specific transport policy measure in a particular scenario, and they do not necessarily include all transportation modes. This gives rise to our first research question.

RQ1 : To what extent can a general-purpose agent-based model of trans- 
port be used to simulate the effects of different policy instruments on travel behavior?

One of the main reasons for the limited use of agent-based models can be the need for a lot of data which is hard to prepare for agent-based models. Nowadays, with the widespread use of pervasive information and communication technologies, travel information is created by various ICT resources such as smart phones. This drives my next research question.

RQ2 : How can transport modeling benefit from opportunities of the new era of digitalization?

One of the important effects of novel ICT services on people's travel behavior is to use online travel planners for choosing route and mode of transportation. This inspires the next research question whether we can use online travel planners as a source of travel information for agentbased models to tackle the challenge of requiring a lot of data.

RQ3 : How can online travel planners be used to produce input data for agent-based simulation of travel behavior?

The fourth research question is driven by the answer to the first question. If we can use online travel planners as an input to the agent-based models what are the advantages and limitations of this approach.

RQ4 : What are the limitations and advantages of using travel planners in simulating effects of policy instruments on travel behavior?

The fifth question is about the validity of our assumption that online travel planners are affecting travel behavior. This means if we use online travel planners as input to an agent-based model for simulating transport policy instruments, the results should conform the real behavior of the travelers.

RQ5 : To what extent can an agent-based model that uses output of online travel planners explain the travel behavior of real passengers in the current situation and predict effects of transport policies? 
Apart from the above-mentioned challenges in data preparation for simulation, there are some usability-related issues that hinder transport planners to use transport simulation systems more effectively. One of these challenges is the complexity of output of simulation systems which makes it hard for decision makers to understand the simulation output and use it for decision making. My last research question is related to the usability of transport simulation systems.

RQ6 : How should the output of transport simulation systems be visualized to improve usability? 


\section{RESEARCH METHOD}

The main research method used in this thesis is design science in the sense that inquiry consists of constructing a new artifact and assessing it. From an engineering standpoint, this is common research practice, typically by designing and building prototypes that are then assessed empirically in order to determine their validity, performance, etc. Hevner et al. [29] present a framework for understanding, executing, and evaluating information systems research. A schematic view of the customized research method adopted from design science process [29] is illustrated in Figure 3.1. The framework includes environment, which defines the problem space and the business needs. In this research the environment refers to the urban transport planning area that includes transport planners, transport policy makers, and transport researchers as the main actors in my research. The other organizational stakeholder of the research is transportation agencies i.e. Skånetrafiken and city of Malmö as the main transport information provider. The last part of the research environment is technological capabilities including digitization opportunities for public transportation (elaborated in Paper 3). The main input from the research environment to design and evaluation of the artifact (simulation system) in my research is study of needs that is part of Paper 5. Study of needs is a survey reflecting the opinions of the research stakeholders about transport policy instruments and their effects on travel behavior in order to understand the needs of a transport policy simulation model. Another crucial input to the design process is applicable knowledge from knowledge base including theories, frameworks, and methods supporting design and evaluation of the simulation system as the main artifact of the design process. An important part of this process is grounding the design of the artifact in the existing body of knowledge in the areas of computer science, transport science, and social science. Paper 1 is a review of previous work in the area of using computational model for evaluating the effects of transport policy instruments.

A design research artifact can actually be any designed object in which a research contribution is embedded in the design. In my research, the artifact is a simulation tool including a simulation model 


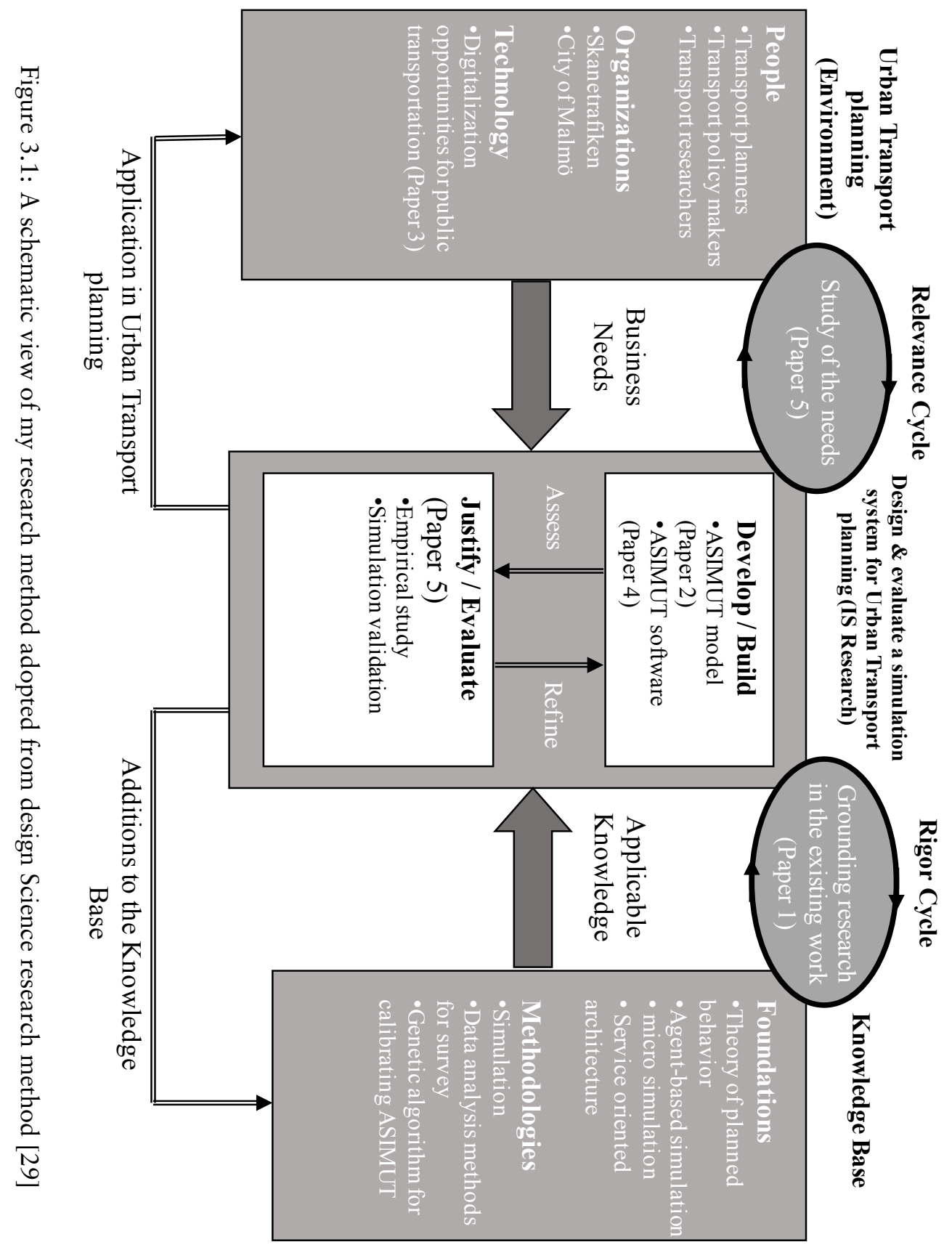


(ASIMUT) presented in Paper 2 and a simulation software explained in Paper 4. Building and evaluating artifacts represent the research activities whereas the resulting constructs, models, methods and instantiating describe the research outputs. The simulation tool is validated in a series of empirical experiments concerning the Malmö-Lund region which is represented in Paper 5. We used a quantitative approach for analyzing the results of simulation experiments to investigate the possible outcomes of changing a specific policy instrument (independent variable) on the choice of travel (dependent variable).

To answer RQ6, we chose an existing transport simulation system (TAPAS) [31] and went through a design science approach to investigate whether visualizing output of the system improves the usability or not. We have distributed questionnaires among transport planners as endusers to collect their requirements. Then we used the existing knowledge in the area of data visualization to design and implement a visualization module for TAPAS. Finally, we evaluated the output of visualization by interviewing end-users The result of this study is presented in Paper 6. 


\section{CONTRIBUTIONS}

In this chapter, we discuss the main theoretical and empirical findings of this thesis that are presented comprehensively in the enclosed papers in Part II of the thesis.

\subsection{A survey on using computational models for evaluating effect of policy instruments}

To understand the research gap in the previous studies on using computational models for analyzing the consequences of policy instruments we have done a survey which is published as Paper 1. The aim of the study was to understand which policy instruments have been investigated, which kinds of models have been used to estimate the effects of policy instruments, and how these models have been applied. This survey showed that despite the recognized potential of agent-based modeling to study behavioral change of a population, it rarely has been used for ex-ante analysis of policy instruments. The result of our survey directed us towards formulating RQ1 in this thesis.

\subsection{Using web services as efficient sources of input to agent-based simulations}

One of the main challenges of using agent-based models in transportation simulation is the need for a huge amount of input data. In order to address RQ3, we have introduced and evaluated a novel approach to tackle this challenge: using web-services such as online travel planners and weather prediction web-services, and etc. The result of my empirical study (presented in Paper 5) revealed that the travel options that are a considerable part of the input data can be generated automatically by online services that can significantly reduce the amount of manual work needed for proving input to the agent-based models. 


\subsection{ASIMUT: a general-purpose model for sim- ulating policy instruments}

As it was mentioned in the problem statement section, only few studies have applied agent-based models to investigate the effect of policy instrument on travel behavior. Even these limited studies have focused on a particular policy. To answer RQ1 in this thesis, we proposed a generalpurpose agent-based model (ASIMUT) to simulate the effect of different policy instruments on travel behavior. The result of using the calibrated model in my empirical study (presented in Paper 5) showed the validity of the ASIMUT model for simulating real scenarios.

ASIMUT is a general-purpose multi-agent based model that can be used for investigating the effects of different types of policy instruments and transport infrastructure investments on travel choices of travelers [28], for example, how the travelers' choices of transport would change in case of introducing a new public transport fare.

In the ASIMUT model, the travelers are explicitly modeled as agents. This enables us to model the decisions of each individual and compute the consequences of their decisions on the transport system. The relevant travel options, for each specific traveler, from an origin (point) A to a destination (point) B are generated. Based on section 1.6, we acknowledge that travelers my use online travel planners to choose travel options. In other words, the model generates part of the choice set by using online travel planners, assuming that travelers consider looking their travel options in travel planners. Obviously, not all possible travel options are available through online travel planners, e.g. combination of cycling and public transport or driving. Therefore, the model generates a bigger choice set by combining different sources of information, trying to mimic how a traveler chooses a travel option in reality. This means ASIMUT is designed in a way that all needed information about travel options can be extracted from online services. The modes of transport included in the model are driving, public transport, cycling, walking, and combinations of public transport, walking and cycling.

Most previous algorithms [21] aim at generating a choice set consist of alternatives that travelers have considered for a given trip. However, some algorithms (e.g. [22]) have used a sampling approach to generate a more rich choice set to avoid source of bias in their model. Our approach in generating choice set for ASIMUT is more close to the latter approach since we iclude all rational possible alternatives by combining data from different web services; however, our approach can potentially introduce a source of bias towards output of online travel planners and web-services that we have used to generate choice set.

In spite of the fact that ASIMUT is an agent-based model, our implementation of ASIMUT is similar to discrete choice models (e.g. [14]) 


$$
\begin{aligned}
& S_{\text {oat }}=W_{\text {cost }} * \operatorname{rel}_{\text {oat }}^{\text {cost }} * \text { val }_{a}^{\text {income }}+W_{\text {time }} * \text { rel }_{\text {oat }}^{\text {time }} * v_{\text {val }}^{\text {workflex }}+W_{\text {conv }} * \\
& \mathrm{rel}_{\text {oat }}^{\text {conv }} * v_{\mathrm{val}}^{\text {age }}+W_{\text {conv }} * \mathrm{rel}_{\text {oat }}^{\text {conv }} * \mathrm{val}_{t}^{\text {wth }}+W_{\text {eco }} * \mathrm{rel}_{\text {oat }}^{\text {envImpact }} * \mathrm{val}_{a}^{\text {eco }}
\end{aligned}
$$

Figure 4.1: ASIMUT traveler behavior model [28]

$$
r e l_{\text {oat }}^{c o n v}=r e l_{\text {oat }}^{\text {wlkDis }}+r e l_{\text {oat }}^{c y c D i s}+\operatorname{rel}_{\text {oat }}^{\text {noofChange }}
$$

Figure 4.2: Definition of the factor 'convenience' [28]

in the sense that in both ASIMUT and discrete choice models there is a utility function that defines the utility of each alternative for selecting the best choice for each specific traveler. However, in the further development we can add other agents such as buses and cars to ASIMUT that enables us to model and study interaction between agents in order to model congestion or interaction between travelers, e.g. carpooling options.

We assume that the choices between alternatives are based on four main factors: the cost of the travel option, the travel time of the alternative, the convenience of the alternative, and the social norm. In the current version of ASIMUT[28], the convenience is defined as a combination of three factors: the number of interchanges, the walking distance and the cycling distance for each travel option. Also, social norms are limited to how much a traveler cares about the environmental consequences of her travel. The amount of $\mathrm{CO}_{2}$ emissions emitted is used as an indicator for how much a specific travel damages the environment.

Furthermore, we believe that the decision making process of the travelers, when choosing between the available travel options is to some extent individual and not the same for all travelers. Therefore, we assume that the best travel option can be different for different travelers according to their characteristics and contextual factors, e.g., weather conditions (weather is represented as val $_{t}{ }^{\text {wth }}$ in Fig. 4.1). The individual characteristics in ASIMUT are age, income, work flexibility, and ecofriendliness. As an example, the perceived value (significance) of the cost factor is different for each traveler and depends on the amount of income of the traveler. We also use other information about travelers such as work and home address, working hours, access to car, and access to bicycle at home and at work, when generating and filtering the relevant travel options.

\subsubsection{A utility function for modeling travel decision}

To model the decision making of travelers, we defined a utility function that is in line with the rational choice approach. We believe ratio- 


$$
\begin{aligned}
\text { rel }_{\text {oat }}^{\text {cost }} & =\frac{\text { Cost }_{\text {oat }}}{\sum_{o^{\prime} \epsilon O} \text { Cost }_{o^{\prime} a t}} \\
\text { rel }_{\text {oat }}^{\text {time }} & =\frac{\text { Time }_{\text {oat }}}{\sum_{o^{\prime} \epsilon O} \text { Time }_{o^{\prime} a t}} \\
\text { rel }_{\text {oat }}^{\text {envImpact }} & =\frac{\text { Co2Emission }_{\text {oat }}}{\sum_{o^{\prime} \epsilon O} \text { Co2Emission }_{o^{\prime} a t}}
\end{aligned}
$$

Figure 4.3: Normalization of cost, time, and environmental awareness [28]

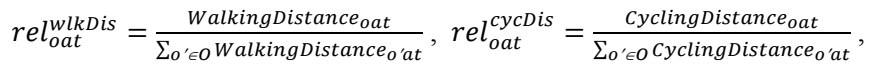

$$
\begin{aligned}
& \text { rel }_{\text {oat }}^{\text {noofChange }}=\frac{\text { NoofChanges }_{\text {oat }}}{\sum_{o^{\prime} \in O^{\prime} \text { NoOfChanges }} o^{\prime} \text { at }}
\end{aligned}
$$

Figure 4.4: Normalization of convenience factors [28]

nal choice is a good enough approach for ASIMUT since online travel planners provide and process all information needed for decision making to the travelers; therefore, rationality of the travelers is less bounded with the limitation of cognitive resources or not having access to all relevant attributes. Moreover, thanks to the online travel planners providing real-time information about potential delays of the public transportation modes, people rely more on the data rather than their bad experience or negative perception. Previous lab experiments [11] also have shown that travelers prefer to rely on the prescriptional information even if the source of information is not reliable. Finally, the result of my empirical study presented in Paper 5 showed that ASIMUT is able to correctly model at least $72 \%$ of real travel decisions made by 313 commuters between two neighbor cities in southern Sweden.

Using a utility function (illustrated in Fig. 4.1), we calculate a score for each of the travel options of the individuals. The factor 'convenience' is determined by combination of the three factors of walking distance, cycling distance, and the number of changes of the travel option o for agent $\mathrm{a}$ in ASIMUT, and it is calculated as shown in Fig. 4.2. The values of relative time and cost are calculated by normalizing the cost and time of a travel option with respect to the other travel options of traveler a for trip t, as shown in Fig. 4.3 in which $O$ refers to the collection of all travel options of trip $t$ for traveler $a$. The same normalization process applies to the factors for convenience, as shown in Fig. 4.4, e.g. in order to calculate the relative environmental impact of a travel option o, the $\mathrm{CO}_{2}$ emission of that travel option is divided by the sum over the $\mathrm{CO}_{2}$ emissions of all the travel options o for trip $\mathrm{t}$ of the agent a. This approach of normalization is chosen in order to remove the unwanted 
effects of large numbers in the data. It should be noted that before the normalization process, we remove non-realistic travel alternatives from the choice set, e.g. biking more than 10 kilometers, or walking between two cities because including unrealistic values in the data can hide the difference in the scores of realistic options. For example, if we include an unrealistic travel option such as walking by 100 kilometers that can take days long, the difference between normalized scores of driving car (e.g. 1 hour) or taking train (e.g. 1.5 hours) will be insignificant that decreases sensitivity of our model for selecting realistic options. In ASIMUT decision making model, the real values for the age, income, work flexibility, environmental awareness (i.e., eco-friendliness), and weather characteristics are transformed to categorical values. These translations are the values used in the utility function, i.e., $v a l_{a}{ }^{\mathrm{xx}}$ and $v a l_{t}{ }^{\mathrm{wth}}$, and they are all numbers between 0 and 1 . It should be noted that the values used in the scoring function are just preliminary estimations; they will be further analyzed and validated in future studies. It should be emphasized here that the calculated score actually represents the disutility of a travel option; therefore, ASIMUT will always choose the travel option with the lowest score among the set of available options for each traveler agent (individual).

\subsubsection{ASIMUT for simulating policy instruments}

ASIMUT is a general-purpose model in the sense that it has the potential to model the effects of various policy instruments and changes in transport infrastructure. When designing the ASIMUT model, two main type of data is used: travelers' characteristics and attributes of different travel options. The availability of these data has lead us to the model design decisions. In design of the ASIMUT model, two main design perspectives have been considered:

1. Using online services as input to the model: ASIMUT is designed in a way to use online services to provide most of the input data. It has the ability to extract network data and information about travel options from online services as opposed to traditional models where the network data is hard-coded to the model. This approach makes the model light-weight and sensitive to any changes in transport network (e.g. price changes, new lines). Figure 4.5 shows how the data needed for the model is captured using online services.

2. Design a general-purpose model: when choosing factors influencing travel behavior we aim at including as many factors as possible in order to ensure the ability of the model to investigate the effects of a variety of policies and policy instruments. However, due to limitations in input data sources which are national travel surveys 


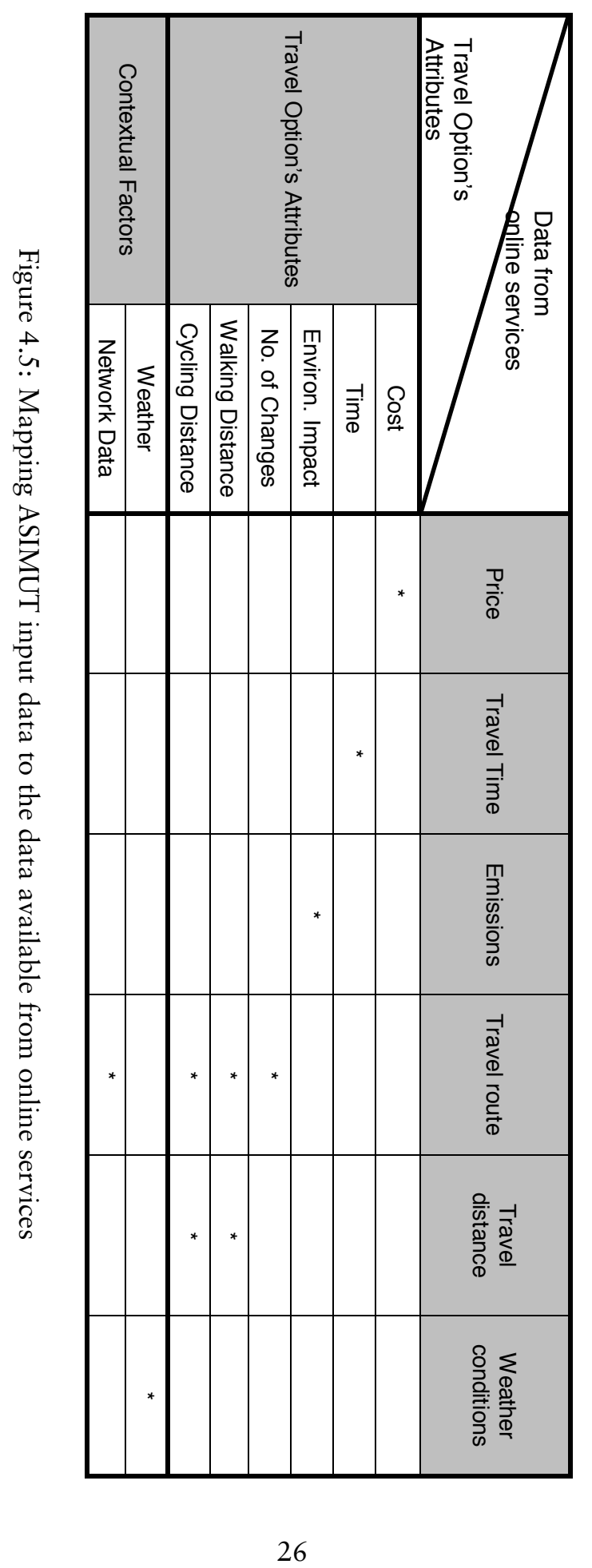


and online travel planners, not all possible factors that influence travel choices can be included, e.g. safety, comfort, etc. Figure 4.6 shows the factors included in the model and how they could potentially support investigating effect of different transport policy instruments on travel behavior.

\subsection{A step-by-step approach for using ASIMUT}

To answer RQ4 and RQ5, we conducted an empirical study where we applied ASIMUT for simulating real world transport scenarios. Our empirical study is explained in Paper 5. This chapter explains a stepby-step method extracted from our empirical study to apply ASIMUT for simulating effects of different policy instruments on travel behavior. The proposed method addresses RQ3. A schematic view of the proposed step-by-step method is illustrated in Figure 4.7.

\subsubsection{Step 1: Requirement analysis}

The first step in simulation of policy instrument is to identify important policy instruments and their effects. In our empirical study, we have distributed an online questionnaire among 21 policy makers and researchers of public transportation in Sweden about the possible requirements of a transport model. The requirements of the transport model is used in the model adoption step to select and customize an appropriate model for simulating policy instruments. In our survey, the respondents are selected based on their expertise and area of work which was relevant to urban transport planning in Sweden. The aim of the questionnaire was to investigate which instruments are the most interesting to study, and what kind of effects are the most important ones to study. The most important policy instruments are used in the simulation step to define scenarios while the most important effects identified in the survey are used to adjust the output of the simulation.

\subsubsection{Step 2: Model adoption}

After identifying the requirements of the model based on the important policy instruments and effects, a transport model should be adopted. Model adoption means to develop a new model, or select and customize an existing model for simulation. Since ASIMUT is developed as a general purpose agent-based model that supports simulation of the effects of various policy instruments, it is recommended to use ASIMUT or a modified version of ASIMUT as the simulation model. The model can be adjusted based on the requirements of the selected policy instruments in Step 1. Table 4.6 that shows which part of ASIMUT is relevant to 


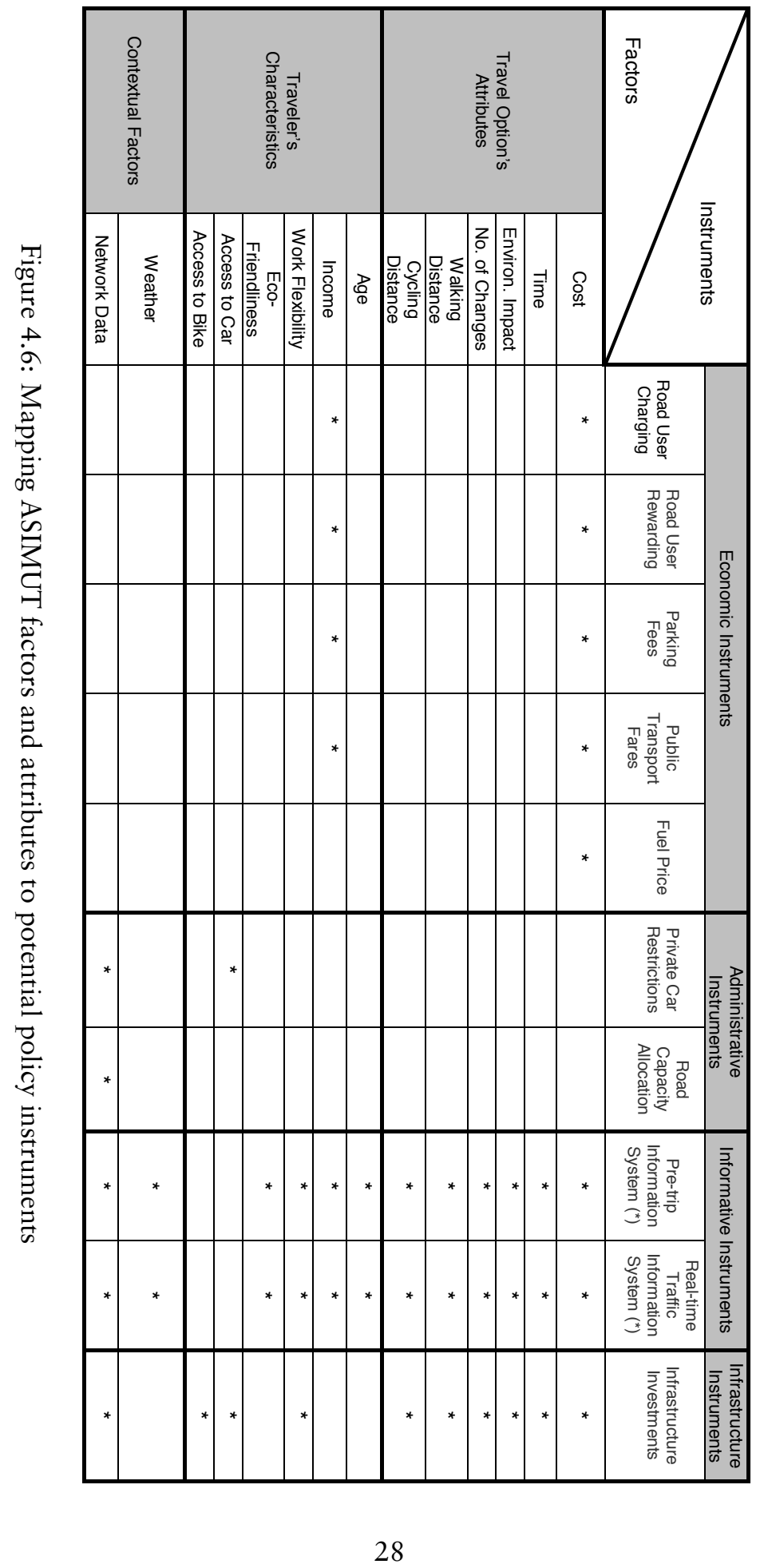




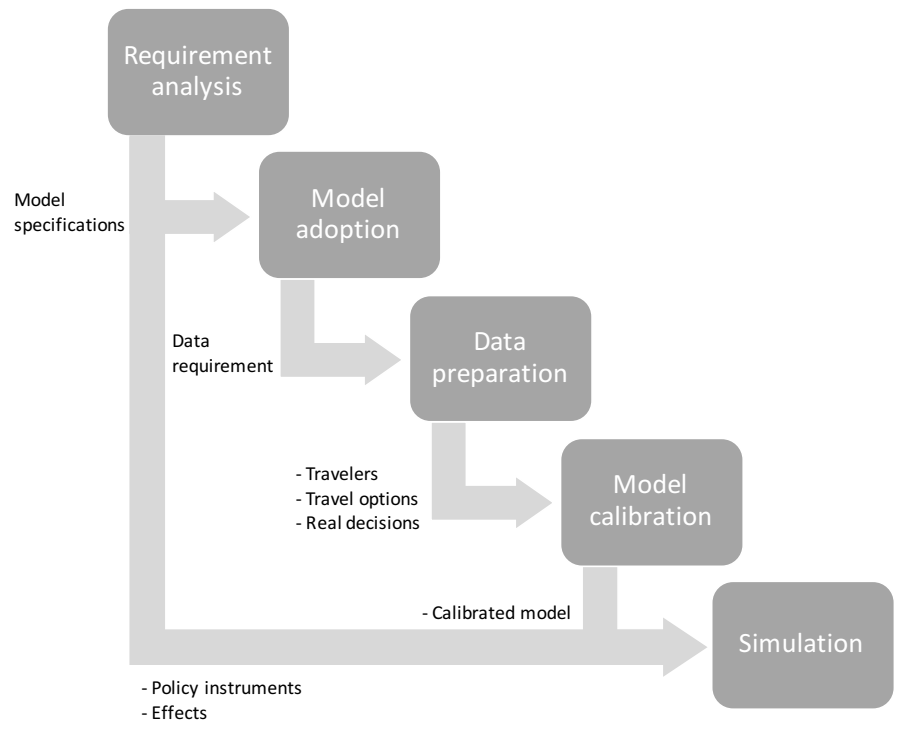

Figure 4.7: A schematic view of the proposed method for using ASIMUT

different policy instruments can be used to modify the model for target policies.

\subsubsection{Step 3: Data preparation}

In this step the data needed for the simulation model needs to be prepared. In agent-based models like ASIMUT the main input data falls into two main categories: 1) traveler agents, 2) travel options. The traveler data includes demographic data of travelers such as age, gender, income, and car ownership, etc. while the travel option descriptions consist of travel cost, time, $\mathrm{CO}_{2}$ emissions, number of changes, etc. as explained in section 4.3.1. The travel agent data can be generated by analyzing national surveys (similar to RVU [38]) or other samples taken from target population. To generate travel option data, it is recommended to use online travel planners and other web services as it is explained in Paper 5. Since this method is limited by the capacity of the online service providers, it is recommended to generate travel options once and record them for running different scenarios. We used a combination of the travel planner provided by Skånetrafiken ${ }^{1}$ and Google Maps Directions web service ${ }^{2}$ to generate travel options.

\footnotetext{
${ }^{1}$ http://labs.skanetrafiken.se/

${ }^{2} \mathrm{https} / /$ developers.google.com/maps/documentation/directions/
} 


\subsubsection{Step 4: Model calibration}

Before we can use any model for predicting the effects of implementing different transport scenarios, the model needs to be calibrated based on the survey data. Calibrating transportation model helps to obtain a close match between the observed and simulated measurements. Transportation simulation models are mathematical abstractions of transportation systems where output is calculated based on particular equations given a specific input data. The main output of the simulation in our model is travel behavior that is defined by decisions that travelers take to choose a travel option. Calibration means adjusting the model's parameters in order to minimize the error $(\varepsilon)$ between output of simulation (Output $t_{\text {sim }}$ ) and observed output in the real world $\left(\right.$ Output $\left._{\text {obs }}\right)$. A mathematical representation of the calibration process is illustrated as follow, in which both output of simulation and the observed output are considered to be a function of the travelers' characteristics and the travel options characteristics.

Output $_{\text {obs }}: f($ Traveler, Travel $) \rightarrow$ Output $_{\text {sim }} \mid$ Traveler, Travel $+\varepsilon$

In our empirical study, we did not have access to details of the real decisions of each individual (i.e. which train or bus line they take); therefore, we used an aggregated data on mode choice to calibrate the model. We used the chosen travel mode (public transport or car) as the observed output for calibrating our simulation model. Due to the complexity of the calibration, we applied genetic algorithm [18] as a heuristic approach which is based on the survival of the fittest candidates to find as good as possible values for the parameters of the travel behavior model that minimizes the error between the output of the simulation and the observed output. We used the single arithmetic crossover method [30] for our crossover function. We started our heuristic approach by defining an initial set of 10 candidates for our 6 parameters. For generation of the next set of parameter values as children, we divided them to two groups. For the first group, we used values of the best parent as input to the crossover function and for the second half of the children, the next best parent is used as input. In order to keep diversity to the next generation of values, we let the last child to be generated by mutation. Then we let the simulation run for couple of days until the error did not change significantly anymore. Using parameters from the best set of candidates, we reached the accuracy of $72 \%$.

\subsubsection{Step 5: Simulation}

In the last step, the scenarios should be defined based on the selected policy instruments from Step 1. Running scenarios that do not demand 
change in the infrastructure is straight forward and fast, the modeler just needs to change the parameter in the model and run the simulation. However, if the infrastructure changes e.g. adding a new line to the public transportation network, the travel options need to be defined and added to the travel options database manually that can be cumbersome and time-consuming. However even in those cases, the travel options that are related to the unchanged part of the infrastructure are still usable that saves a lot of time and manpower to produce all needed travel options. The output of the simulation needs to cover the important effects identified in the requirement analysis step.

\subsection{A visualization tool for transportation sim- ulation}

In order to answer RQ6, we have implemented a visualization tool for an existing transportation simulation system called TAPAS [31]. In this study that is explained in Paper 6, we have analyzed the visualization related requirements through a user-centric approach. The visualization tool was implemented based on the identified requirements. We evaluated the usability of the visualization tool by sending out an online questionnaire to the users of the simulation system together with screenshots of the visualization output. 


\section{CONCLUSIONS}

Reviewing previous studies on using computational models for analyzing the effect of transport policies on transportation systems shows that agent-based models have not been used much in spite of their great potential for simulating dynamic aspects of policy instruments and travel behavior. The main reason can be the need for a lot of data which is hard to prepare for the modeler. This has led to limited use of agentbased models in previous studies and even in those studies the scope of simulation is limited to only particular scenarios. In this thesis, we proposed a general-purpose agent-based simulation model for urban transportation that supports simulation of a wide range of policy instruments. The proposed model is designed in a way that a large part of the input data can be generated automatically using online web-services. This novel approach tackles the challenge of agent-based simulation as a dataintensive method. This can lead to more widespread use for agent-based simulation in solving complex and realistic transportation scenarios.

In this part, the research questions are briefly answered as follows.

RQ1: To what extent can a general-purpose agent-based model of transport be used to simulate the effects of different policy instruments on travel behavior?

We proposed ASIMUT as a general-purpose model for simulating the effect of various policy instruments on travel behavior in Paper 2 . As Figure 4.6 shows a mapping between components of the ASIMUT model to various policy instruments, all of policy instruments identified in my survey (mentioned in Paper 1) can be simulated using ASIMUT. However, for simulating some of the policy instruments such as informative instruments that are more general, the ASIMUT model needs to be modified or developed based on the specific requirements of the policy instrument. For example, if in a hypothetical scenario the policy makers wish to investigate the effect of providing information about the environmental impact of the travels through an information system to the travelers, ASIMUT can be used without any modification. However, for investigating the effect of using e.g. a real-time traffic congestion sys- 
tem on travel behavior, ASIMUT needs to be developed to include the real-time traffic data.

RQ2: How can transport modeling benefit from opportunities of the new era of digitalization?

Our explorative study presented in Paper 3 revealed that there are great opportunities for both transport operators and planners, as well as for the travelers in the fourth wave of digitalization, also referred to as the Internet of Things (IoT). One of the most prominent opportunities for transport modeling is to use online travel planners for preparing input data of transport simulation in agent-based modeling which is a very data intensive procedure.

RQ3: How can online travel planners be used to produce input data for agent-based simulation of travel behavior?

Section 4.4 and Paper 5 explain a step-by-step method for applying ASIMUT and using online travel planners to produce travel options. In short, the travel agent data needs to be generated based on national surveys or other samples from target population while the travel options and its associated information can be automatically generated using a combination of online travel planners and other web-services. My empirical study (explained in Paper 5) has shown that this approach saves a lot of time and energy from modelers to run different simulation scenarios.

RQ4: What are the limitations and advantages of using travel planners in simulating effects of policy instruments on travel behavior?

Results of our empirical study (Paper 5) showed that using online travel planners to prepare input data for agent-based simulation can be a very efficient and a rather fast approach to run scenarios (like scenarios 2 and 3 in this paper) that do not require change in the infrastructure. Total time to run simulation for our first and second scenarios was about 4 minutes on a normal Macbook computer. However, to get the travel options from online travel planners such as Google Maps API in our case, we need time, computational resources, and of course budget to pay for requesting a high amount of data. But when the data is collected, it can be reused to run different scenarios.

RQ5: To what extent can an agent-based model that uses output of online travel planners explain the travel behavior of real passengers in the current situation and predict effects of transport policies?

Calibration of the ASIMUT for 313 commuters between Lund and Malmö during a period of 10 days has led to $72 \%$ accuracy (discussed 
in Paper 5). This means that ASIMUT is able to model at least $72 \%$ of real travelers' decisions correctly. The results of simulating hypothetical scenarios also seemed to be acceptable. It should be noted that we calibrated the model according to the choice of mode of the travelers on one single day from RVU data. However, in ASIMUT we have included travels of a traveler agent over a period of 10 days in order to investigate the effect of weather and different timetables. We have used the same main mode of transport of traveler in the RVU survey for calibrating our model. The lack of travel information in the RVU survey for travelers in different days with various weather conditions and time tables, might be one source of error in calibration. Moreover, the RVU survey has been done over a period of 5 weeks, so that a various weather condition has affected travel choices during this 5 weeks which is not recorded in the survey. Finally, in the RVU data it is not recorded if the traveler uses any online travel planner applications for planning his/her trip or not. If some of the travelers do not use online travel planners their decision might be based on other reasons rather than output of online travel planners that can negatively affect the accuracy of the calibration.

RQ6: How should the output of transport simulation systems be visualized to improve usability?

The results of implementing and evaluating a visualization tool for TAPAS [31] (Paper 6) indicated that showing aggregated results related to all simulation scenarios is very useful. Moreover, visualizing simulation results for each route is more useful than visualizing them for links; even though link-based data can also be useful in some special cases. The visual representation of simulation data is evaluated much more useful than non-interactive and paper-based solutions (e.g. tables and Excel sheets) for communicating the simulation results. Finally, the use of colors in the bar charts and the possibility to customize the simulation scenario to adjust the output accordingly was suggested as additional improvements to the proposed visualization tool.

\subsection{Future work}

ASIMUT can be validated through more case studies in other geographical areas or/and by applying other policy instruments. Moreover, ASIMUT can be further developed in order to include more factors influencing travel behavior, e.g. safety, comfort. This development needs more research on travel behavior modeling as well as high quality input data about the travelers. The model can benefit from using the new generation of travel surveys that are based on collecting data from mobile phones. Using a more detailed travel survey as input to the model, can increase the quality of the results and opens opportunities for investi- 
gating more complicated policy instruments due to availability of more detailed data about travelers and their travels. In addition, ASIMUT can be used as a tool for transportation policy makers where they define different scenarios that do not require change in the infrastructure such as changing fuel price, public transportation fare, etc. In that case, the usability of ASIMUT can be evaluated and improved regarding the learnability, understandability, ease of use, and etc. Finally, the accuracy of calibrating ASIMUT can be improved by modeling uncertainty in different aspects of the system. 


\section{BIBLIOGRAPHY}

[1] Staffan Algers, Lars-Göran Mattsson, Clas Rydergren, and Bo Östlund. Sampers : erfarenheter och utvecklingsmöjligheter på kort och lång sikt. Technical report, KTH, Centre for Transport Studies, CTS, 2009. TSC import 734 2012-01-30. QC 20120507. 5

[2] Sanghyuk An, JX Cui, and LY Li. Agent-based approach to model commuter behaviour's day-to-day dynamics under pre-trip information. Intelligent Transport Systems, IET, 5(1):70-79, 2011. 8, 12,13

[3] ARRB Group Limited. KonSULT-Developing an International Knowledgebase on Urban Transport Policy Instruments, 2002. 4

[4] Jacqueline Augusiak, Paul J Van den Brink, and Volker Grimm. Merging validation and evaluation of ecological models to 'evaludation': a review of terminology and a practical approach. Ecological Modelling, 280:117-128, 2014. 4

[5] Jang Won Bae, Euihyun Paik, Kiho Kim, Karandeep Singh, and Mazhar Sajjad. Combining microsimulation and agent-based model for micro-level population dynamics. Procedia Computer Science, 80:507-517, 2016. 6

[6] Michael Balmer, Marcel Rieser, Konrad Meister, David Charypar, Nicolas Lefebvre, Kai Nagel, and K Axhausen. Matsim-t: Architecture and simulation times. Multi-agent systems for traffic and transportation engineering, pages 57-78, 2009. 9

[7] David Banister. The influence of habit formation on modal choice? a heuristic model. Transportation, 7(1):5-33, 1978. 9

[8] Steven C Bankes. Agent-based modeling: A revolution? Proceedings of the National Academy of Sciences, 99(suppl 3):7199-7200, 2002. 7 
[9] Ana LC Bazzan and Franziska Klügl. A review on agent-based technology for traffic and transportation. The Knowledge Engineering Review, 29(3):375-403, 2014. 7

[10] Moshe E Ben-Akiva and Steven R Lerman. Discrete Choice Analysis: Theory and Application to Travel Demand, volume 9. MIT Press, 1985. 9

[11] Eran Ben-Elia, Roberta Di Pace, Gennaro N Bifulco, and Yoram Shiftan. The impact of travel information?s accuracy on routechoice. Transportation Research Part C: Emerging Technologies, 26:146-159, 2013. 11, 24

[12] Eric Bonabeau. Agent-based modeling: Methods and techniques for simulating human systems. Proceedings of the National Academy of Sciences, 99(suppl 3):7280-7287, 2002. 7

[13] Peter Bonsall. Traveller behavior: Decision-making in an unpredictable world. In Intelligent Transportation Systems, volume 8, pages 45-60. Taylor \& Francis, 2004. 9

[14] John Bowman. Incorporating bicycling into activitybased regional travel forecasting models in denmark: Identified needs and proposed solutions. www.jbowman.net, Feb 2014. 22

[15] Arnaud Burgess, T.Ming Chen, Maaike Snelder, Nils Schneekloth, Artem Korzhenevych, Eckhard Szimba, Markus Kraft, Michael Krail, Otto Anker Nielsen, Christian Overgård Hansen, Angelo Martino, Davide Fiorello, and Panayotis Christidis. Final report trans-tools (tools for transport forecasting and scenario testing) deliverable 6. TNO INRO, Delft, The Netherlands, 2008. 5

[16] Jerker Denrell and James G March. Adaptation as information restriction: The hot stove effect. Organization Science, 12(5):523538, 2001. 10

[17] Hussein Dia. An agent-based approach to modelling driver route choice behaviour under the influence of real-time information. Transportation Research Part C: Emerging Technologies, 10(5):331-349, 2002. 8

[18] Omar Baqueiro Espinosa. A genetic algorithm for the calibration of a micro-simulation model. arXiv preprint arXiv:1201.3456, 2012. 30

[19] Dick Ettema and Harry Timmermans. Costs of travel time uncertainty and benefits of travel time information: conceptual model and numerical examples. Transportation Research Part C: Emerging Technologies, 14(5):335-350, 2006. 11 
[20] Gunnar Flötteröd, Yu Chen, and Kai Nagel. Behavioral calibration and analysis of a large-scale travel microsimulation. Networks and Spatial Economics, 12(4):481-502, 2012. 7

[21] E. Frejinger and M. Bierlaire. On path generation algorithms for route choice models. In Choice modelling: the state-of-the-art and the state-of-practice, pages 307-315. Emerald Group Publishing Limited, 2010. 22

[22] E. Frejinger, M. Bierlaire, and M. Ben-Akiva. Sampling of alternatives for route choice modeling. Transportation Research Part B: Methodological, 43(10):984 - 994, 2009. 22

[23] Gerd Gigerenzer, Reinhard Selten, et al. Rethinking rationality. Bounded rationality: The adaptive toolbox, 1:12, 2001. 9

[24] Nigel Gilbert and Klaus Troitzsch. Simulation for the social scientist. McGraw-Hill Education (UK), 2005. 6, 7, 13

[25] Dominik Grether, Yu Chen, Marcel Rieser, Ulrike Beuck, and Kai Nagel. Emergent effects in multi-agent simulations of road pricing. In Proceedings of the Annual Meeting of the European Regional Science Association (ERSA), 2008. 8

[26] Banafsheh Hajinasab, Paul Davidsson, Johan Holmgren, and Jan A Persson. On the use of on-line services in transport simulation. Transportation Research Procedia, 21:208-215, 2017. 11

[27] Banafsheh Hajinasab, Paul Davidsson, and Jan A Persson. A survey on the use of computational models for ex ante analysis of urban transport policy instruments. Procedia Computer Science, 32:348$355,2014.11,13$

[28] Banafsheh Hajinasab, Paul Davidsson, Jan A Persson, and Johan Holmgren. Towards an agent-based model of passenger transportation. In Lecture Notes in Computer Science, Multi-Agent Based Simulation XVI, volume 9568, pages 132-145. Springer, 2015. 22, 23, 24

[29] Alan R. Hevner, Salvatore T. March, Jinsoo Park, and Sudha Ram. Design science in information systems research. MIS Q., 28(1):75105, March 2004. 17, 18

[30] John Henry Holland. Adaptation in natural and artificial systems: an introductory analysis with applications to biology, control, and artificial intelligence. MIT press, 1992. 30 
[31] Johan Holmgren, Paul Davidsson, Jan A. Persson, and Linda Ramstedt. Tapas: A multi-agent-based model for simulation of transport chains. Simulation Modelling Practice and Theory, 23:1 - 18, 2012. 19, 31, 35

[32] Andreas Horni, Kai Nagel, and Kay W Axhausen. The multi-agent transport simulation MATSim. Ubiquity Press London, 2016. 9

[33] Emmanuel Kemel and Corina Paraschiv. Prospect theory for joint time and money consequences in risk and ambiguity. Transportation Research Part B: Methodological, 56:81-95, 2013. 11

[34] Hans Kremers, Peter Nijkamp, Piet Rietveld, et al. The scope of meta-analysis for transport policy impact analysis in environmental economics. Comparative Environmental Economic Assessment, pages $47-73,2002.5$

[35] Todd Litman. What's it worth: Economic evaluation for transportation decision-making. In Internet Symposium on Benefit-Cost Analysis Transportation Association Of Canada, 2001. 4

[36] Anthony D May, Charlotte Kelly, and Simon Shepherd. The principles of integration in urban transport strategies. Transport Policy, 13(4):319-327, 2006. 4

[37] Simon McDonnell and Moira Zellner. Exploring the effectiveness of bus rapid transit a prototype agent-based model of commuting behavior. Transport Policy, 18(6):825-835, 2011. 8, 12, 13

[38] Mats Nyfjäll, Pär Wikman, Charlotte Wahl, and Martin Ullberg. resvaneundersökning i skåne 2013 - malmö stad. http://malmo.se/download/18.50dab45f146afe8fc2c25f6/ 1491301439661/RVU2013Sk\%C3\%A5ne+1.0.pdf, Jun 2014. Accessed: 2017-09-30. 29

[39] Joseph N Prashker and Shlomo Bekhor. Route choice models used in the stochastic user equilibrium problem: a review. Transport reviews, 24(4):437-463, 2004. 9

[40] Steven F Railsback and Volker Grimm. Agent-based and individual-based modeling: a practical introduction. Princeton university press, 2012. 6, 7

[41] Linda Ramstedt. Transport policy analysis using multi-agent-based simulation. PhD thesis, Department of Systems and Software Engineering, School of Engineering, Blekinge Institute of Technology, 2008. 8, 13 
[42] Jeppe Rich, Johannes Bröcker, Christian Hansen Overgård, Artem Korzenewych, Otto Anker Nielsen, and Goran Vuk. Report on scenario, traffic forecast and analysis of traffic on the ten-t, taking into consideration the external dimension of the union: Trans-tools version 2; model and data improvements. Technical report, Technical University of Denmark, 2009. 5

[43] Georgina Santos, Hannah Behrendt, and Alexander Teytelboym. Part ii: Policy instruments for sustainable road transport. Research in transportation economics, 28(1):46-91, 2010. 4

[44] Oliver Schöller-Schwedes. The failure of integrated transport policy in germany: a historical perspective. Journal of Transport Geography, 18(1):85-96, 2010. 4

[45] Herbert Alexander Simon. Models of bounded rationality: Empirically grounded economic reason, volume 3. MIT press, 1982. 9

[46] Anthony M. Starfield, Karl Smith, and Andrew L. Bleloch. How to Model It: Problem Solving for the Computer Age. McGraw-Hill, Inc., New York, NY, USA, 1993. 4

[47] Dusan Teodorovic. Transport modeling by multi-agent systems: a swarm intelligence approach. Transportation planning and Technology, 26(4):289-312, 2003. 13

[48] Nikolas Thomopoulos, Moshe Givoni, et al. ICT for transport: Opportunities and threats. Edward Elgar Publishing, 2015. 10

[49] Amos Tversky and Daniel Kahneman. Advances in prospect theory: Cumulative representation of uncertainty. Journal of Risk and uncertainty, 5(4):297-323, 1992. 9

[50] Joachim Wahle, Ana Lúcia C Bazzan, Franziska Klügl, and Michael Schreckenberg. The impact of real-time information in a two-route scenario using agent-based simulation. Transportation Research Part C: Emerging Technologies, 10(5):399-417, 2002. 8 


\section{PAPERS}



I 

Paper 1: A survey on the use of computational models for ex ante analysis of urban transport policy instruments

\title{
Authors:
}

Banafsheh Hajinasab, Paul Davidsson, Jan A. Persson

\section{Published:}

Procedia Computer Science 32 (2014): 348-355.

\begin{abstract}
This paper provides an analysis of the use of computational models for predicting the effects of different policy instruments on urban transport systems. We define a framework for describing and comparing current approaches that takes into account (i) what policy instruments are studied, (ii) what type of effects are predicted, (iii) which factors are modeled, (iv) the type of model used, and (v) what data sources are used. The main conclusions of the analysis are: (i) despite the recognized potential of agent-based modeling to study behavioural change of a population, it rarely has been used for ex-ante analysis of policy instruments using real-world data, (ii) some factors that influence travellers' decisions, such as comfort and departure time, have not been considered much in the modeling, (iii) ex-ante analysis of economic instruments constitute the majority of studies, but informative instruments have been recently considered due to the increasing use of information technology in transportation.
\end{abstract}




\title{
A survey on the use of computational models for ex ante analysis of urban transport policy instruments
}

\author{
Banafsheh Hajinasab $^{\mathrm{a} *}$, Paul Davidsson ${ }^{\mathrm{a}}$, Jan A. Persson ${ }^{\mathrm{a}}$
}

${ }^{a}$ Department of Computer Science, Malmö University, Malmö, Sweden

\begin{abstract}
This paper provides an analysis of the use of computational models for predicting the effects of different policy instruments on urban transport systems. We define a framework for describing and comparing current approaches that takes into account (i) what policy instruments are studied, (ii) what type of effects are predicted, (iii) which factors are modeled, (iv) the type of model used, and (v) what data sources are used. The main conclusions of the analysis are: (i) despite the recognized potential of agent-based modeling to study behavioural change of a population, it rarely has been used for ex-ante analysis of policy instruments using real-world data, (ii) some factors that influence travellers' decisions, such as comfort and departure time, have not been considered much in the modeling, (iii) ex-ante analysis of economic instruments constitute the majority of studies, but informative instruments have been recently considered due to the increasing use of information technology in transportation.
\end{abstract}

(C) 2014 Published by Elsevier B.V. This is an open access article under the CC BY-NC-ND license (http://creativecommons.org/licenses/by-nc-nd/3.0/).

Selection and Peer-review under responsibility of the Program Chairs.

Keywords: survey; policy instruments; computational models; travel behaviour; ex ante analysis

\section{Introduction}

There is a wide set of policy instruments that can be used for designing transport strategies in order to achieve transport system objectives. Examples of such objectives are reduction of congestion, private vehicle use, emissions, or more generally, travel demand management. Policy instruments range from more conventional instruments such as land use regulation, vehicle regulation, infrastructure investment, and pricing schemes, to newer instruments such

\footnotetext{
* Corresponding author. Tel.: +0-000-000-0000 ; fax: +0-000-000-0000

E-mail address: author@institute.xxx
} 
as application of information technology to improve resource allocation and service quality, as well as attitudinal changes [1]. The policy instruments are very different in nature and have different effects on different transport systems. Moreover, the combination of policy instruments might have a totally different effect on the transport system compared to applying each individual instrument alone [2]. It has been argued that the development of sustainable transport strategies often fails due to lack of integration of different policy instruments $[3,4]$.

In order to know which policy instruments to choose in a particular situation, it is important not only to have knowledge about availability of policy instruments, but also their potential impact on the transport system. There are different methods to analyze the effects of policy instruments. Kremers et al. [5] have categorized methods for transport policy analysis into two main types: qualitative ad-hoc approaches that are solely based on expert judgment or interviews, and quantitative structured approaches, where a statistical or econometric model based on quantified data is used. Ad-hoc approaches are typically used in situations where there is no possibility for a structured approach due to time constraints, non-repetitive situations, or lack of data. We will here focus on structured, or quantitative approaches that have used computational models to estimate the effects of policy instruments.

In the project KonSULT, an international knowledge base on the impact of different urban transport policy instruments was developed $[1,6]$. It provides an online decision-support tool that can assist policy makers in developing urban transport strategies. While this work has some similarities with our research, there are also fundamental differences. We do not aim to develop a comprehensive encyclopedia of policy instruments, but rather focus on the use of computational models for impact assessment of policy instruments.

In the present work we have analyzed the applications of computational models to estimate the effects different policy instruments that we have found in the literature. The aim is to understand which policy instruments have been investigated, which kinds of models have been used to estimate the effects of policy instruments, and how these models have been applied. To do this in a systematic way, we have developed a framework for characterizing the applications. In order to develop this framework, we have extracted the common characteristics of different published studies in the area of ex ante analysis of urban transport policies. We have collected a set of papers through online databases of peer-reviewed articles. We first conducted an exploratory, opportunistic literature review in which the set of policy instruments have been studied was identified, followed by a more focused literature search where we gathered the relevant studies investigating each of the policy instruments.

\subsection{Scope}

In this survey, we have focused on policy instruments that aim to change the behaviour of travellers, rather than to change the behaviour of transport system planners. That is, we do not regard policy instruments, such as regulative requirements on regional planning or public transport tendering. In addition, our focus is on instruments that have direct effects on the travel behaviour. Thus, we exclude instruments such as vehicle tax, which affect longterm travel decisions e.g. concerning what type of car to buy.

Furthermore, we will not include instruments that concern infrastructure development and network expansion. The motivation for this is that in the recent years, the focus of urban transport system planning has shifted from building new infrastructure to more efficient use of the existing infrastructure [7]. In particular, investments on road infrastructure are usually very expensive and time consuming, and above all, it is often impossible to find the space needed due to the high density of cities and land-use regulations.

As mentioned earlier in this paper, we limit our search to those papers that have used computational models to estimate the effects of policy instruments.

\section{Survey framework}

In this section we describe the framework that has been used to characterize the applications of computational models to estimate the effects of policy instruments.

\subsection{Policy instruments}

Below we describe the policy instruments investigated in the studies we have reviewed. 
- Road user charging (RUC) is a common name for different methods to collect money from road users [8]. The motivation for introducing RUC is often either to make the road users compensate for the costs caused by their transports such as road wear and accidents, i.e., internalization of external costs, or to change the behaviour of the road users to be consistent with the objectives of the transport policies, e.g. to reduce congestion [9], and/or emission [10]. There are different approaches to implement RUC such as road link-based, cordon/area-based, time-based, and distance-based charging. The distance-based approach computes the charges based on the distance traveled in a specified area, while the road link approach computes the charge based on the number of times a vehicle crosses a specific link. Cordon-based charges are calculated in a similar way as road link charges, but in this case based on the number of times the vehicle enters an area, referred to as the cordon. In case of timebased charging, the time spent in a specified area provides the basis for calculating charges. In addition, the specific time period in which the travel is happening can determine the level of charges.

- Road User Rewarding (RUW) is closely related to RUC, but in this case the travellers will be awarded by money or other kinds of incentives instead of punishment. Charging and rewarding can alternatively be called as push and pull strategies [11]. Rewarding is claimed to be more acceptable and effective as it makes people happier, thus increasing the effectiveness of people. However, there are at least two disadvantages to be considered which might affect the effectiveness of rewarding. First and foremost, charging provides revenues, which can be used to enhance the transportation services in order to increase social welfare, whereas rewarding does not. Secondly, it is argued that losses have more emotional impact than gains, which makes them more effective [11].

- Parking fees refer to the charges related to on-street or off-street parking of a vehicle. Parking fees either can be sensitive to the time of the day, or not. In general, parking fees seem to be more acceptable for drivers than other kinds of policies since they have been used for a long time [12].

- Public transport fares concern the cost that travellers need to pay in order to use public transport services. Public transport here refers to the passenger transport services provided by a 'third party' and available for use by all members of the community [13] including buses, metro, ferries, light rail, subways, commuter rail, and regional or inter-urban rail [14].

- Fuel price comprises the actual cost of the fuel and the fuel tax determined by the government. Governments can either pursue fuel subsidization policies or increase fuel taxes to control people's transportation behaviour $[15,16]$.

- Private car restrictions correspond to the rules and regulations that the government may impose in order to decrease attractiveness and possibility of private car usage. It can be in the form of some restrictions on the car use such as time, zone and distance restrictions, or speed limits, as well as rules for car ownership [17,18].

- Road capacity allocation often concerns introduction of bus priority policies such as Bus Rapid Transit (BRT) systems. BRT operates on exclusive lanes of existing roads and provides flexible and high performance service, which is comparable to light rail transit or metro systems but to a much lower cost [19]. Compared to the traditional busses, BRT systems reduce travel time and hence increase the attractiveness of public transport [20].

- Pre-trip planning support is an information-provisioning instrument in which the traveller can obtain useful information before the actual travel. The purpose is to make the travellers more informed before deciding on mode, route, and departure time, or even whether to travel or not. Examples of pre-trip information are public transport schedules and travel time estimations. The pre-trip information can be obtained via smartphones, radio channels, television, and online services [21]. Advanced Traveller Information Systems (ATISs) are an example of information source for travellers that provide travellers with pre-trip, as well as real-time information [22].

- Real-time traffic information is the provision of information about the current traffic situation to the travellers, which can be used to adapt the travel behaviour, e.g. route choice or departure time $[23,24,25]$. The real-time information can be provided through en-route guidance on the road, e.g. variable message signs, or ATISs [26]. According to [27] the policy instruments fall into three main categories: Economic, Administrative, and Informative. Using this classification, we can summarize the above-mentioned policy instruments as illustrated in Figure 1. 


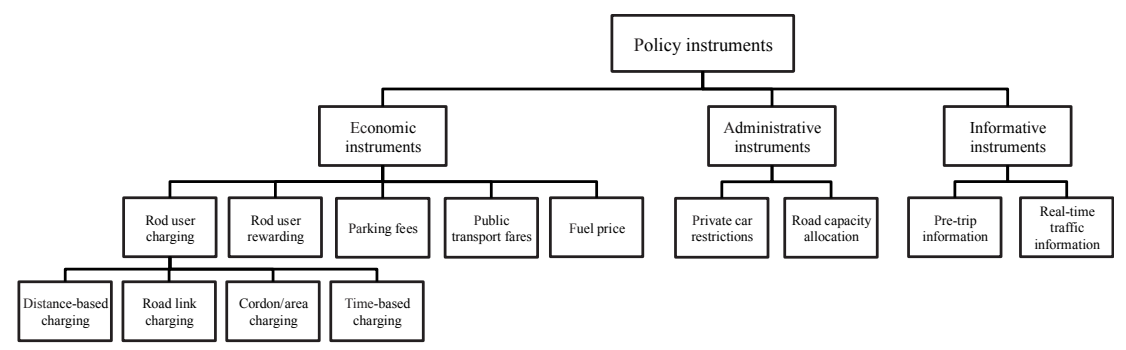

Figure 1. Categorization of policy instruments in this study.

\subsection{Travel behaviour effects}

This aspect concerns how the policy instruments influence traveller behaviour. More specifically, it deals with how policy instruments play a part in people's travel-related choices. The following effects have been studied in the articles reviewed:

- Mode choice: changes in the selection of transportation mode, e.g. bus, train, car, bicycle, etc.

- Route choice: changes in which route is taken.

- Travel time: changes to the time duration of the travel, from origin to destination.

- Departure time: changes to the time when the traveller leaves the origin and starts traveling.

- Destination choice: changes in the travellers' choice of destination.

- Parking choice: changes to where the travellers park their cars.

\subsection{Factors}

This aspect is referring to the factors that are considered in modeling of travellers' decision making. The factors we have identified in the studied literature fall into the following categories:

- Time: covers all the time-related factors which have been included in the models such as expected travel time, departure time, and time of the year in which travel happening (e.g. season), etc.

- Cost: all the cost-related factors fall into this category, e.g. in-vehicle cost, cordon cost, parking fee, congestion charge, emission charge, fuel cost, tolls, public transport cost, etc.

- Socio-demographic characteristics: includes characteristics of the travellers such as age, gender, income, education level, household structure, vehicle type, car availability, driving license ownership, public transport pass ownership, personality characteristics (e.g. strong habits or environmental awareness), etc.

- Trip purpose: the motive for the travel such as work, education, leisure, shopping, or even mix of them.

- Comfort: includes characteristics related to the traveller's comfort during a travel such as number of transfers, number of stops, availability of seats, in-vehicle crowding, out of vehicle facilities, personal security, safety, cleanness and temperature inside vehicle, etc.

\subsection{Model}

As was mentioned in the introduction, our focus in this paper is quantitative methods of impact assessment of policy instruments. We have different types of models in the literature. A majority of the studies have applied different kinds of discrete choice models such as multinomial logit model (MNL), mixed logit model (MXL), binary logit model (BIL), nested logit model (NLM), probit model, alternative-specific discrete choice model, multiple regression analysis, and logistic regression. Some studies have applied agent-based simulation models in which the travellers' interaction are taken into account, while others are based on conventional simulation methods [28]. There 
are some studies that have combined different models, such as [35] in which an integrated model of MNL and agent based simulation have been used.

\subsection{Data sources}

This aspect deals with the origin of the data that is fed into the models. Data can be acquired by several ways, including national surveys and censuses, stated preferences surveys, and revealed preferences. In a stated preference survey, the travellers are asked to provide their preferences and choices in different situations, whereas in revealed preference experiments, the empirical observation of travellers' choices is considered as source of information [29]. Data from national surveys may be less valid for the particular area and situation studied.

\subsection{Size of the modelled area}

We have characterized the study areas according to their population as follows: Very small city (less than 100,000), Small city (100,000-1 million), Medium-sized city ( $1-5$ million), Large city (5-10 million), and Megacity (more than 10 million) inhabitants.

\section{Discussion}

The results of the survey are summarized in Table 1. In this section, we will analyze the results in terms of the different aspects of the survey framework, starting with the type of policy instrument studied.

It can be observed that it is the effects of economic policy instruments that have been analyzed the most in the literature. One explanation for this finding can be the fact that the cost-related instruments have been the most widely used in the past and they are still very common in travel demand management. Therefore, it is natural that the policy makers wish to focus on ex-ante analysis of the economic instruments. Another reason could be that travellers' decisions based on cost are easier to model than those based on information availability etc. Looking into the year of publication reveals that the economic instruments have been evenly considered in the literature from the past till nowadays. However, the type of instrument has changed over the time and new ways of road-user charging or public transport fare regulations have been studied. Moreover, we can see that the administrative instruments have only recently been considered. Another interesting observation is that the informative instruments have been mostly investigated in the period of 2001-2002 and then after 2011. The introduction of new information systems and the growing trend towards application of new ITS services in the beginning of 2000 may account for this result.

We observe that changes in mode choice and route choice are the most commonly studied effects of policy instruments on people's travel behaviour. Also change in departure time has been studied rather frequently, but slightly less than route choice. We note that the changes in travel time are seldom regarded, as well as changes in where to travel and park.

Concerning the factors considered in modeling, socio-demographic characteristics, travel time and cost are the most frequently considered, whereas factors such as trip purpose, time of the year, departure time and comfort are seldom considered. In particular, we found that in almost all cases some socio-demographic characteristics of the study population have been considered. Among socio-demographic characteristics, age, gender, income, work schedule flexibility and car availability are the most used factors, while vehicle type and residential location are the least used. Although it has been argued that comfort and convenience of travellers during a journey can have significant effects on travellers' choices (especially mode choice) [30], they are typically not considered. This may be due to the difficulty of gathering data about comfort and travellers attitudes because these factors have subjective constructs [31].

The majority of studies have used some kind of discrete choice model, in particular logit models have been the most frequently used. We notice that the use of agent-based simulation models has increased in recent years. Due to high computational power requirement of these kinds of models and difficulty of collecting disaggregated data, these models have been considered difficult to implement and therefore were not very commonly used in the past. 


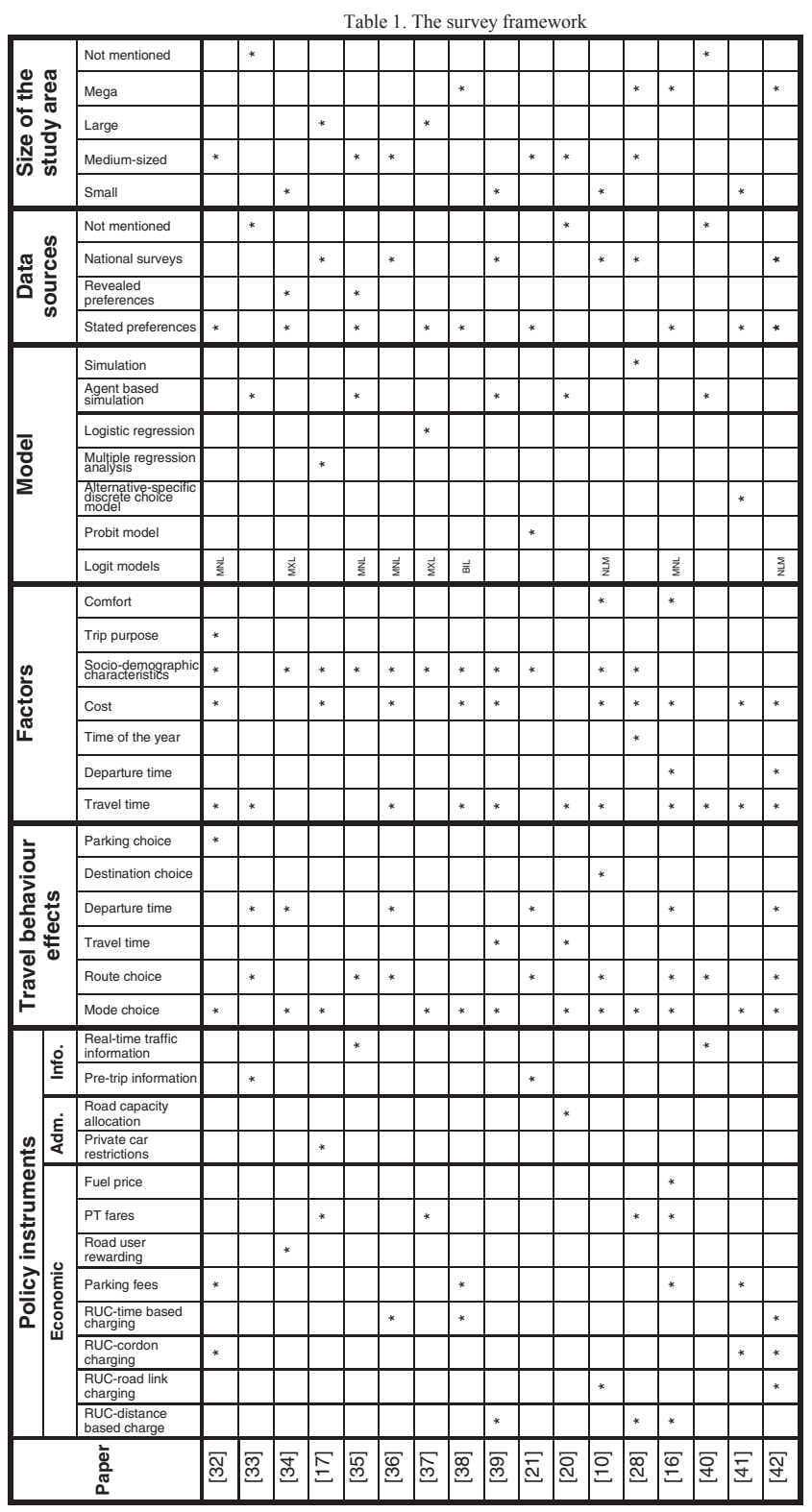


However, the conventional discrete choice models have been criticized for neglecting the interaction effects and oversimplification which can introduce significant biases in output [43].

The data required for the models is mainly acquired through stated preferences surveys, whereas revealed preferences surveys were used only in two cases. There are also some studies that have used artificial data. It can be seen from the table that all the studies that have used artificial data, use agent-based simulation for impact analysis. It can be due to the fact that agent-based simulation method requires a lot of detailed data about the network and population that might not be easy to acquire.

Medium-sized cities (1-5 million inhabitants) are dominant in the studied models, just as many as large and mega cities taken together, whereas cities smaller than 1 million have been less studied.

\section{Conclusions and future work}

Based on the review, we conclude that mathematical-based and discrete choice models have been considered as the main method for ex-ante analysis of policy instruments for many years. However, due to oversimplification of discrete choice models in considering interaction effects, the results from these models are often inaccurate especially in real-world problems where the interactions of policies/people are high [43]. Therefore, we argue that there is a need to develop agent-based models that use real-world data. In particular, such models may be useful for smaller cities, which to date often have not been the target for policy instrument studies although they often have major traffic problems. Moreover, there are some policy instruments used in reality that to the best of our knowledge have not been studied using computational models for ex-ante analysis, e.g. travel awareness campaigns, and ITSbased travel ticketing systems. We think that it is worth investigating whether also these instruments could be analyzed using computational models.

\section{References}

[1] May, A. D., \& Taylor, M. A. (2002). KonSULT-Developing an International Knowledgebase on Urban Transport Policy Instruments (Doctoral dissertation, Bureau of Transport and Regional Economics).

[2] May, A. D, Kelly, C., Shepherd, S. (2006). The principles of integration in urban transport strategies. Transport Policy, 13(4), $319-327$.

[3] Schöller-Schwedes, O. (2010). The failure of integrated transport policy in Germany: a historical perspective. Journal of Transport Geography, 18(1), 85-96.

[4] Santos, G., Behrendt, H., \& Teytelboym, A. (2010). Part II: Policy instruments for sustainable road transport. Research in Transportation Economics, 28(1), 46-91.

[5] Kremers, H., Nijkamp, P., \& Rietveld, P. (2002). The scope of meta-analysis for transport policy impact analysis in environmental economics. Comparative Environmental Economic Assessment, Edward Elgar, Cheltenham, UK, 47-73.

[6] KonSULT, the Knowledgebase on Sustainable Urban Land use and Transport. (2013). Retrieved December 27, 2013, from http://www.konsult.leeds.ac.uk/public/level1/sec09/

[7] Illenberger, J., Flotterod, G., \& Nagel, K. (2007). Enhancing matsim with capabilities of within-day re-planning. In proceedings of $10^{\text {th }}$ International IEEE Conference on Intelligent Transportation Systems (ITSC 2007), IEEE (94-99).

[8] Johansson, B., \& Mattsson, L. G. (1995). Principles of road pricing. Road pricing: Theory, empirical assessment and policy. Springer Netherlands, 7-33.

[9] Eliasson, J., Börjesson, M., van Amelsfort, D., Brundell-Freij, K., \& Engelson, L. (2013). Accuracy of congestion pricing forecasts. Transportation Research Part A: Policy and Practice, 52, 34-46.

[10] Mishra, S., \& Welch, T. F. (2012). Joint Travel Demand and Environmental Model to Incorporate Emission Pricing for Large Transportation Networks. Transportation Research Record: Journal of the Transportation Research Board, 2302(1), 29-41.

[11] Bliemer, M. C., \& van Amelsfort, D. H. (2010). Rewarding instead of charging road users: a model case study investigating effects on traffic conditions. European Transport, 44, 23-40.

[12] Albert, G., \& Mahalel, D. (2006). Congestion tolls and parking fees: A comparison of the potential effect on travel behavior. Transport Policy, 13(6), 496-502.

[13] D'Este, G., Taylor, M. A. P., \& Radbone, I. G. (1994). Demand responsive public transport for Australia: 1. The trade-offs. In Papers of the Australian Transport Research Forum, 1994, Vol 19. 
[14] Glover, L. (2011). Public Transport as a Common Pool Resource. In proceedings of the 34th Australasian Transport Research Forum (ATRF), 28 - 30 September 2011, Adelaide, Australia.

[15] Khoo, H. L., Ong, G. P., \& Khoo, W. C. (2012). Short-term impact analysis of fuel price policy change on travel demand in Malaysian cities. Transportation Planning and Technology, 35(7), 715-736.

[16] Vrtic, M., Schuessler, N., Erath, A., \& Axhausen, K. W. (2007). Route, Mode, and Departure Time Choice Behaviour in the Presence of Mobility Pricing. In proceedings of Transportation Research Board 86th Annual Meeting (No. 07-1618).

[17] de Grange, L., Troncoso, R., \& González, F. (2012). An empirical evaluation of the impact of three urban transportation policies on transit use. Transport Policy, 22, 11-19.

[18] Massot, M. H., \& Armoogum, J. (2003). Speed and car traffic regulation in urban areas. IATSS research, 27(2), 46-55.

[19] ITDP, Bus rapid transit planning guide. (2007). Retrieved December 28, 2013, from http://www.itdp.org/microsites/bus-rapid-transitplanning-guide

[20] McDonnell, S., \& Zellner, M. (2011). Exploring the effectiveness of bus rapid transit a prototype agent-based model of commuting behaviour. Transport Policy, 18(6), 825-835.

[21] Jou, R. C. (2001). Modeling the impact of pre-trip information on commuter departure time and route choice. Transportation Research Part B: Methodological, 35(10), 887-902.

[22] Zito, P., Amato, G., Amoroso, S., \& Berrittella, M. (2011). The effect of Advanced Traveller Information Systems on public transport demand and its uncertainty. Transportmetrica, 7(1),31-43.

[23] Ben-Elia, E., \& Shiftan, Y. (2010). Which road do I take? A learning-based model of route-choice behaviour with real-time information. Transportation Research Part A: Policy and Practice, 44(4), 249-264.

[24] Jou, R. C., Kitamura, R., Weng, M. C., \& Chen, C. C. (2008). Dynamic commuter departure time choice under uncertainty. Transportation Research Part A: Policy and Practice, 42(5), 774-783.

[25] Jou, R. C., Hensher, D. A., \& Chen, K. H. (2007). Route Choice Behaviour of Freeway Travellers Under Real-time Traffic Information Provision-Application of the Best Route and the Habitual Route Choice Mechanisms. Transportation Planning and Technology, 30(6), 545570.

[26] Bonsall, P. (1992). The influence of route guidance advice on route choice in urban networks. Transportation, 19(1), 1-23

[27] Trafikverket. (2012). Styrmedel för ett effektivare transportsystem, Trafikverket, 2012:106.

[28] Tsekeris, T., \& Voß, S. (2010). Public transport and road pricing: a survey and simulation experiments. Public Transport, 2(1-2), 87-109.

[29] Wardman, M. (1988). A comparison of revealed preference and stated preference models of travel behaviour. Journal of Transport Economics and Policy, 71-91.

[30] Stet, M., \& Rosu, A. (2012). PSPC (Personal, Social, Psychological, Cultural) factors and effects on travel consumer behviour. Esonomics and Management, 17(4), 1491-1496.

[31] Diana, M. (2010). From mode choice to modal diversion: A new behavioural paradigm and an application to the study of the demand for innovative transport services. Technological Forecasting and Social Change, 77(3), 429-441.

[32] Ahmadi Azari, K., Arintono, S., Hamid, H., \& Rahmat, R. A. O. (2013). Modelling demand under parking and cordon pricing policy. Transport Policy, 25, 1-9.

[33] An, S., Cui, J. X., \& Li, L. Y. (2011). Agent-based approach to model commuter behaviour's day-to-day dynamics under pre-trip information. IET Intelligent Transport Systems, 5(1), 70-79.

[34] Ben-Elia, E., \& Ettema, D. (2011). Rewarding rush-hour avoidance: A study of commuters' travel behaviour. Transportation Research Part A: Policy and Practice, 45(7), 567-582.

[35] Dia, H. (2002). An agent-based approach to modelling driver route choice behaviour under the influence of real-time information. Transportation Research Part C: Emerging Technologies, 10(5), 331-349.

[36] Eluru, N., Paleti, R., \& Bhat, C. R. (2010). Examining the Influence of Tolls on Commute Departure and Route Choice Behaviour in the Chicago Region. Southwest Regional University Transportation Center, Center for Transportation Research, the University of Texas at Austin, (No. SWUTC/10/169200-1).

[37] Fabbro, Y. (2013). Free public transport: a socio-cognitive analysis. (Master's thesis, Hasselt University, Hasselt, Belgium). Retrieved from http://hdl.handle.net/1942/15542

[38] Feng, S. W., \& Ye, J. J. (2008). A Comparative Study of Parking and Congestion Charge Policies based on Transport Mode Choice Estimation. ITSC 2008. In proceedings of $11^{\text {th }}$ International IEEE Conference on Intelligent Transportation Systems (ITSC 2008), IEEE (506-510).

[39] Grether, D., Chen, Y., Rieser, M., Beuck, U., \& Nagel, K. (2008). Emergent effects in multi-agent simulations of road pricing. In proceedings of Annual meeting of the European Regional Science Association ERSA, 8.

[40] Wahle, J., Bazzan, A. L. C., Klügl, F., \& Schreckenberg, M. (2002). The impact of real-time information in a two-route scenario using agent-based simulation. Transportation Research Part C: Emerging Technologies, 10(5), 399-417.

[41] Washbrook, K., Haider, W., \& Jaccard, M. (2006). Estimating commuter mode choice: A discrete choice analysis of the impact of road pricing and parking charges. Transportation, 33(6), 621-639.

[42] De Palma, A., \& Lindsey, R. (2006). Modelling and evaluation of road pricing in Paris. Transport Policy, 13(2), 115-126.

[43] Takama, T., \& Preston, J. (2008). Forecasting the effects of road user charge by stochastic agent-based modelling. Transportation Research Part A: Policy and Practice, 42(4), 738-749. 

II 

Paper 2: Towards an agent-based model of passenger transportation

\title{
Authors:
}

Banafsheh Hajinasab, Paul Davidsson, Jan A. Persson, Johan Holmgren

\section{Published:}

In International Workshop on Multi-Agent Systems and AgentBased Simulation, pp. 132-145. Springer, Cham, 2015.

\begin{abstract}
In this paper, we present a multi-agent based simulation model for supporting the decision making in urban transport planning. The model can be used to investigate how different transport infrastructure investments and policy instruments will affect the travel choices of passengers. We have identified four main categories of factors influencing the choice of travel: cost, time, conven- ience, and social norm. However, travelers value these factors differently de- pending on their individual preferences, something that can be modeled in an agent-based model. Moreover, instead of modeling the transport system explic- itly, on-line web services are used to generate travel options. The model can support transport planners by providing modal share, as well as economical and environmental consequences. As a first step towards validation of the model, we have conducted a simple case study of three scenarios where we analyze the effects of changes to the public transport fares on commuter's travel choices in the Malmö-Lund region in Sweden.
\end{abstract}




\title{
Towards an agent-based model of passenger transportation
}

\author{
Banafsheh Hajinasab ${ }^{*}$, Paul Davidsson, Jan A. Persson, Johan Holmgren \\ Department of Computer Science, Malmö University, Sweden \\ \{banafsheh.hajinasab, paul.davidsson, jan.a.persson, \\ johan.holmgren\} @mah.se
}

\begin{abstract}
An agent-based simulation model for supporting the decision making in urban transport planning is presented. The model can be used to investigate how different transport infrastructure investments and policy instruments will affect the travel choices of passengers. We identified four main categories of factors influencing the choice of travel: cost, time, convenience, and social norm. However, travelers value these factors differently depending on their individual characteristics, such as age, income, work flexibility and environmental engagement, as well as on external factors, such as the weather. Moreover, instead of modeling the transport system explicitly, online web services are used to generate travel options. The model can support transport planners by providing estimations of modal share, as well as economical and environmental consequences. As a first step towards validation of the model, we have conducted a simple case study of three scenarios where we analyze the effects of changes to the public transport fares on commuters' travel choices in the Malmö-Lund region in Sweden.
\end{abstract}

Keywords: multi-agent based simulation, traveler behavior modeling, passenger transport, impact assessment, web services

\section{Introduction}

The design of a "greener" transport system can be supported by a wide set of transport measures, including both transportation policy instruments and investments in infrastructure, such as new public transport pricing schemes, taxes and fares for motorized transport, new bus stops and lines, and new parking space.

In this paper, we propose a novel agent-based simulation model for supporting decision making in urban transport planning. The model, which we refer to as ASIMUT (Agent-based simulator for urban passenger transport), can be used to investigate how different transport measures affect the decisions of the travelers. It takes into account how factors like cost, time, convenience, and social norm influences the decisions on an individual level depending on the socio-economical features of the individual. Another innovative property of the simulator is that it makes use of online web ser-

adfa, p. 1, 2015.

(C) Springer-Verlag Berlin Heidelberg 2015 
vices in order to generate travel options, rather than modeling the transport system explicitly.

In the next section we review the related work and motivate the chosen agentbased approach. Section 3 presents ASIMUT. To make a first validation of the model, a simple case study of three scenarios is presented in section 4, where we analyze the effects of changes to the public transport fares on commuter's travel choices in a region of Sweden. Some concluding remarks are provided in section 5.

\section{Related work and motivation}

As the application of transport measures may have substantial impact on the travelers' behavior, it is very important to assess their impact before implementation, so that negative effects can be avoided and positive effects can be confirmed. One way of doing this is to perform experimental studies in the real world, but such studies are often very expensive and time-consuming. A common approach for assessing the effects of transport measures is to use computational models, which allows studying the transport system in a simulated environment. A recent review of policy impact assessment models concludes that conventional discrete choice models are the dominating method for travel behavior modeling [8]. These traditional models operate on highly aggregated data. Moreover, they are typically built to study transport in a particular country or a region, and they are often based on the so-called four-step modeling approach. The four steps are: trip generation, where the frequency of trips between zones is determined; trip distribution, where origins are matched with destinations; mode choice, where the proportion of trips between each origin and destination that use a particular transport mode is computed; and route assignment, where all trips are assigned to routes. However, four-step models have been criticized both for neglecting the interaction effects between the involved actors and for oversimplification, which often lead to significant biases in output, especially in settings where the interaction between policies and/or travelers is significant [17]. Furthermore, these models only take into account a limited number of the factors influencing travel behavior [8].

Agent-based simulation modeling is another approach that has been used for impact assessment of transport measures. It is often regarded as a bottom-up approach where each traveler is treated as an interacting, autonomous and independent entity. Thus, it differs from conventional top-down approaches that focus on overall aggregated analysis of the system's behavior $[6,18]$.

In the agent-based simulation model presented in this paper, the passengers are modeled as agents. We generate the different travel alternatives of an agent using existing web services of online travel planners. We consider both motorized and nonmotorized modes of transportation and the combinations of them in generating travel alternatives. The model focuses on how to travel when the destination is already decided, i.e., corresponding to steps 3 and 4 of the traditional four-step models. More specifically, we focus on the mode choice, route choice and departure time choices of travelers, when source and destination data is available from the traveler agent, i.e., the traveler's home and work addresses. We believe that significant improvements to 
these steps can be made using a more detailed bottom-up approach, and that this can be used together with any approach to determine the travel demand.

The agent-based modeling approach provides a more dynamic approach with respect to the level of detail in modeling different parts. For instance, more interesting parts of the infrastructure can be modeled with a higher granularity. This makes it possible to study the effects of, e.g., building a new bike parking facility that is safe and efficient and close to a train station, or allowing the travelers to bring their bikes on the trains. Furthermore, by using an agent-based method it is possible to model what travel options different travelers actually are aware of, or consider, when deciding what option to choose. This makes it possible to study the effects of, e.g., travel awareness campaigns and the availability of advanced travel planning systems. Such interventions are difficult, or even impossible, to study using traditional models.

Furthermore, agent-based models are able to capture time-related aspects, such as the effects of synchronization and optimization of timetables [16]. There are many transport policy measures that concern time, e.g., time-differentiated congestion and parking fees. Such transport policies are difficult to study using traditional models, but they may have an important influence on travel choices.

We further argue that the use of an agent-based modeling approach, which captures the behaviors of travelers and their interactions between each other and with the environment, will facilitate capturing each individual's preferences and characteristics. This is critically important in order to determine the actual decisions of individual travelers. Thus, agent-based modeling seems very well suited to predict and analyze the effects of different transport measures, since it explicitly models the decisions of each individual and is able to compute the consequences of these decisions. It should be noted that agent-based modeling might require more information about travelers on an individual level than the traditional models, which to a large extent are based on population averages. However, modern consumer technology like smartphones, as well as ITS services like advanced ticketing and tracking systems based on "Internet of Things" technology (connected devices), enable efficient, large-scale, collection of individual travel data.

There are few studies that have applied an agent-based modeling approach in the context of transport policy analysis [8]. In most cases, the agent-based models have been very simple and do not realize the potential of the approach [3,14]. These models are mostly developed to investigate the effects of a specific transport measure concerning a specific scenario. Furthermore, they do not include all relevant modes of transportation. The input variables, the model construction, and the collected output are very much chosen with a specific scenario in mind. Therefore, these models cannot investigate the effects of various kinds of transport measures in different scenario settings. This means that they are unable to be used as a decision support system to support transport policy making. An agent-based model that bears some resemblance with the one we propose was developed by Grimaldo et al. [7]. It takes into account cost, travel time and environmental in determining travel choice, but it does not regard convenience and makes no difference between individuals (age, income, etc.) except for car-ownership. Moreover, the transport system modeled is very simplistic, 
e.g., just one road and two travel options, either car or train. In particular, combined transport modes, such as walking, biking, car, bus, and train, are not at all considered.

There are also frameworks for implementing large-scale agent-based transport simulations, e.g. MATSim [4], but they focus on traffic flows and vehicles rather than travel option choices and travelers.

The majority of the traditional models are mode choice models [13], which aim to answer how many travelers will switch to another mode of transport in case of any change in transport system [2]. However, in addition to the choice of transport mode, there are also other important aspects of travel behavior, such as route choice and departure time choice [11]. In order to have a comprehensive and accurate impact assessment, we claim there is a need to investigate the impact on all aspects of travel.

\section{ASIMUT}

In the proposed model, each passenger is modeled as an agent. This enables us to include each individual's preferences and characteristics into the travel choice modeling. The decision-making process of travelers when choosing between the available travel alternatives is to some extent individual and not the same for all travelers. This means that there is no objectively optimal travel choice from point A to point B for all travelers in a given situation. Therefore, we assume that the "best" travel alternative can be different for different travelers. In ASIMUT, the choices between alternatives are based on four main factors: cost, time, convenience, and social norm. The perceived value (priority) of each of these factors is typically different for each traveler and depends on:

- The traveler's characteristics; refers to the attributes of each traveler and have an important influence on the choice of travel. Examples include socio-economic attributes and geographical location of home and workplace.

- The available travel options at the time of travel and their related cost, travel time, $\mathrm{CO}_{2}$ emission, number of changes, and walking and cycling distance.

- Contextual factors, factors related to the context where the travel happens, e.g. the current and predicted weather.

Web-services are used in ASIMUT for data collection. We generate the travel alternatives for a traveler from point $\mathrm{A}$ to point $\mathrm{B}$, using the web services provided by online travel planners. The use of online travel planners for generating travel alternatives is a novel approach which enables us to capture the most recent information about route alternatives and their relevant characteristics such as cost and travel time. Furthermore, it provides the model with real-time information that adapts automatically with updates, e.g., if the bus schedules change, this change will be automatically updated in ASIMUT. Due to recent developments in application of information systems for online trip planning, nowadays most travelers have access to online travel planners and are able to retrieve almost all the possible travel alternatives at the time of departure. Therefore, we believe integration of web services of online travel planners in ASIMUT makes the model represent the real traveling behavior and is highly con- 
sistent with the way travelers choose to travel in everyday life. We use the route alternatives' data gathered from web services as input in the decision-making model.

\subsection{Passenger behavior modeling}

For modeling the individual's travel decision-making we use the theory of planned behavior, which is an extension of the theory of reasoned action [1]. It assumes that humans are rational and they make systematic use of information available to them while they also consider the implications of their actions before they decide for a certain behavior. In ASIMUT, we consider cost, travel time, and convenience as the rational factors that affect the choice of travel. A rational agent aims to maximize the utility and hence minimize cost and travel time and maximize convenience.

However, travelers do not always act completely rational. Social norms and personal values may affect the choice of travel. The theory of planned behavior complements the theory of reasoned action by adding the concept of social norm [1]. Environmental awareness of the travelers is modeled as a social norm in ASIMUT. The theory of planned behavior has also the possibility to cover the behaviors that are not fully under an individual's volitional control. This is very important in travel decision-making where the choice of travel by each individual is not only influenced by her characteristics, attitudes, and subjective norms, but also on intervening environmental conditions, such as the weather which we have included as a contextual factor in ASIMUT.

As mentioned earlier, we use four main categories of factors when making travel choices: cost, time, convenience, and social norms. The significance of each of these factors is determined by each traveler's individual characteristics and contextual factors. In ASIMUT, the value of each of these factors is calculated based on traveler's characteristics and weather conditions. It has been argued that the factors influencing choice of travel can be valued differently for different travel purposes [9,7]. We have included a weight for each of the factors (i.e., cost, time, convenience, $\mathrm{CO}_{2}$ emission) in order to be able to change the significance of each factor for different travel purposes. These weights will also be used for calibration purposes. For the decisionmaking model, we use the weighted sum model [19].

The traveler characteristics that we include in ASIMUT are: age, income, work flexibility, environmental awareness i.e. eco-friendliness, work and home address, working start and end times, access to car, and access to bicycle at home and work. We use work and home address, working hours, access to car, and access to bicycle at home and work directly when generating the travel alternatives, while the other mentioned factors are used for choosing between different travel alternatives. In Table 1 we describe a model of how all these factors can potentially affect the choice of travel and how they interrelate. The main factors influencing travel behavior are listed as columns in Table 1, while the rows are referring to traveler's characteristics and contextual factors. We believe that the income level of the traveler can affect the traveler's perception of travel costs. Therefore, in the proposed decision making model, we use this concept to calculate the value of cost for each traveler; the higher income decreases the influence of the cost on the travel decision of the traveler [5,13]. For 
calculating the value of time, we use the traveler's work flexibility factor. We assume that more flexible working hours decreases the value of travel time to some extent.

Johansson et al. show that travelers who are more environmentally conscious tend to take the travel options that have less negative effects on the environments, or more specifically, the travel options that generate least $\mathrm{CO}_{2}$ emissions [12]. Therefore, in ASIMUT we assume that the amount of $\mathrm{CO}_{2}$ emission can affect the individual's choice of transport, depending on the individual's level of eco-friendliness.

We assume that convenience is comprised of walking distance, cycling distance, and number of changes for a travel option. The number of changes is defined as the number of transfers between vehicles in order to complete a journey. It has a negative effect on the choice of a travel option; the more interchanges in a travel option, the less convenient it is perceived [10]. Moreover, the number of changes of a travel option makes it less attractive the older you are [15]. Furthermore, we assume that the interchange between vehicles is less convenient in case of bad weather conditions.

Heinen et al. [9] reviewed the factors influencing cycling and indicated that there is a relationship between age and cycling, although it is not universal. While most studies have concluded that the willingness to bike decline with age, there are also some other studies that have not found any significant relation between age and cycling. Weather has also a high influence on the distance the individuals are willing to cycle. High precipitation and low temperature have been found as the most significant weather conditions influencing cycling level. There appears to be no significant relation between the other factors (e.g., income) and cycling [9]. In ASIMUT, we assume that convenience is more important for older travelers. Moreover, bad weather conditions (e.g., rain, snow, or low temperature) decrease the convenience of travel options with long walking distance, cycling distance, and higher number of changes.

Table 1. Interrelationship between the factors influencing choice of travel

\begin{tabular}{|c|c|c|c|c|c|c|c|}
\hline \multirow{2}{*}{ Factors } & \multicolumn{7}{|c|}{ Travel option's attributes } \\
\cline { 3 - 8 } & Cost & Time & Environ. impact & \multicolumn{3}{c|}{ Convenience } \\
\cline { 3 - 8 } & $\begin{array}{c}\text { Travel } \\
\text { costs }\end{array}$ & $\begin{array}{c}\text { Travel } \\
\text { time }\end{array}$ & $\mathrm{CO}_{2}$ emission & $\begin{array}{c}\text { No. of } \\
\text { changes }\end{array}$ & $\begin{array}{c}\text { Walking } \\
\text { distance }\end{array}$ & $\begin{array}{c}\text { Cycling } \\
\text { distance }\end{array}$ \\
\hline \multirow{2}{*}{$\begin{array}{c}\text { Traveler's } \\
\text { characteristics }\end{array}$} & Age & & & & $*$ & $*$ & $*$ \\
\cline { 2 - 8 } & Income & $*$ & & & & & \\
\cline { 2 - 8 } & Work flex. & & $*$ & & & & \\
\cline { 2 - 8 } & Eco-friend & & & $*$ & & $*$ & $*$ \\
\hline $\begin{array}{c}\text { Contextual } \\
\text { Factor }\end{array}$ & Weather & & & & $*$ & $*$ & \\
\hline
\end{tabular}

\subsection{Decision-making model}

We use a utility function in order to calculate a score for each travel option. The factors influencing travel behavior are the main components of the model. The values of these components are a function of the characteristics of the traveler (i.e., age, income, work flexibility, and eco-friendliness), and contextual factor (i.e. weather). It should be emphasized here that the calculated score actually represents the disutility 
of a travel option; therefore, an agent will always choose the travel option with the lowest score among the set of available options.

The components of the scoring function have different scales and unit of measurements, and some are quantitative (e.g., age and income), while the others are qualitative or categorical (e.g., weather and work flexibility). In order to avoid domination of larger values, make the components consistent, and neutralize the unit of measurement of the values, we chose to normalize the attributes of the travel options; corresponding to the columns in Table 1. These normalized attributes are referred as relative values in the equation (1), e.g., rel oat $_{\text {envimp }}$, which refers to the relative environmental impact of travel option $o$ for agent $a$. The relative values are typically different for different agents, since these values are calculated with respect to the travel options available for a specific agent. Moreover, we have converted all the characteristics of the travelers and contextual factors to categorical data. These values are called as $v a l_{a}^{x x}$ in the equation (1), where $x x$ are the factors of the traveler $a$ mentioned in the rows of Table 1 and $v a l_{t}^{w t h}$ is the value assigned to the weather conditions of trip $t$. As we discuss further below, all val $a_{a}^{x x}$ and $v a l_{t}^{w t h}$ are assigned values in the range $[0,1]$.

As mentioned earlier, we chose to assign a weight to each factor, i.e., $W_{\text {cost }}, W_{\text {time }}$, $W_{\text {conv }}, W_{\text {envimpact }}$ refering to the weight of cost, time, convenience, and environmental impact, respectively. These weights are mainly used for calibration, but they can also be used in order to change the importance of each factor according to travel motive, e.g. traveling to work or travel for leisure. The score $S_{\text {oat }}$ (i.e., disutility) for travel option $o$ for agent $a$ and trip $t$ is calculated as:

$$
\begin{aligned}
& S_{\text {oat }}=W_{\text {cost }} * \operatorname{rel}_{\text {oat }}^{\text {cost }} * v_{\text {val }}^{\text {income }}+W_{\text {time }} * \operatorname{rel}_{\text {oat }}^{\text {time }} * v_{\text {a }}^{\text {workflex }}+W_{\text {conv }}^{\text {work }} * \\
& \operatorname{rel}_{\text {oat }}^{\text {conv }} * v_{\text {val }}^{\text {age }}+W_{\text {conv }} * \operatorname{rel}_{\text {oat }}^{\text {conv }} * v_{\text {val }}^{\text {wth }}+W_{\text {envImpact }} * \operatorname{rel}_{\text {oat }}^{\text {envImpact }} * v_{\text {al }}^{\text {eco }}
\end{aligned}
$$

As mentioned earlier, convenience is determined by the three factors of walking distance, cycling distance, and the number of changes of the travel option $o$ for agent $a$ in ASIMUT, and it is calculated as:

$$
r e l_{\text {oat }}^{c o n v}=r e l_{\text {oat }}^{\text {wlkDis }}+r e l_{\text {oat }}^{c y c D i s}+r e l_{\text {oat }}^{\text {noofChange }}
$$

The relative time and cost are calculated by normalizing the cost and time of a travel option with respect to the other travel options of traveler $a$ for trip $t$. In the below equations, $O$ refers to the collection of all travel options of trip $t$ for traveler $a$, i.e.,

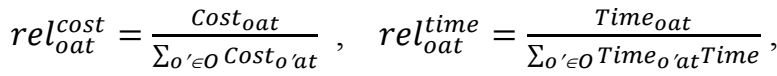

$$
\begin{aligned}
& \text { rel }_{\text {oat }}^{\text {envImpact }}=\frac{\text { Co2Emission }_{\text {oat }}}{{\sum o_{o^{\prime} \in O} \text { Co2Emission }_{o^{\prime} a t}}_{\text {Eat }}}
\end{aligned}
$$

The factors for convenience are also normalized, as shown below. For example, in order to calculate the relative environmental impact of a travel option $o$, the $\mathrm{CO}_{2}$ emission of that travel option is divided by the sum over the $\mathrm{CO}_{2}$ emissions of all the travel options $o$ for trip $t$ of the agent $a$ : 
rel $_{\text {oat }}^{\text {wlkis }}=\frac{\text { WalkingDistance }_{\text {oat }}}{\sum_{o^{\prime} \in O} \text { WalkingDistance }_{o^{\prime} a t}}$, rel $_{\text {oat }}^{\text {cycDis }}=\frac{\text { CyclingDistance }_{\text {oat }}}{\sum_{o^{\prime} \in O} \text { CyclingDistance }_{o^{\prime} a t}}$,

rel $_{\text {oat }}^{\text {noofChange }}=\frac{\text { NoOfChanges }_{\text {oat }}}{\sum_{o^{\prime} \in O} \text { NoOfChanges }_{o^{\prime} a t}}$

As part of the decision-making model, we translate the real values for the age, income, work flexibility, environmental awareness (i.e., eco-friendliness), and weather characteristics, into categories as shown in the Table 2 (in the value column). These translations are the values used in the disutility function, i.e., $v a l_{a}^{x x}$ and $v a l_{t}^{w t h}$, and they are all numbers between 0 and 1 . As an illustrative example, for $v_{a l}^{a g e}$ we translate an income higher than 100000 SEK to $v_{a l}^{a g e}=0.1$, an income in the range $[50000,10000]$ to the $v a l_{a}^{a g e}=0.3$, etc. It can be seen that $v a l_{a}^{a g e}$ increases as the income level decreases, which means that the travel cost will be valued lower for the higher income level of the travelers. It should be noted that the values used in the scoring function are just preliminary estimations; they will be further analyzed and validated in future studies.

Table 2. The categorization of characteristics of travelers and contextual factor $\left(\right.$ val $_{a}^{x x}$ or val $\left._{t}^{\text {wth }}\right)$

\begin{tabular}{|c|c|c|}
\hline Variable & Range & Value \\
\hline \multirow{4}{*}{ Age } & $15-25$ & 0.1 \\
\cline { 2 - 3 } & $25-35$ & 0.3 \\
\cline { 2 - 3 } & $35-55$ & 0.5 \\
\cline { 2 - 3 } & $55-70$ & 0.7 \\
\hline \multirow{4}{*}{ Income (monthly) } & +70 & 0.9 \\
\cline { 2 - 3 } & +100000 & 0.1 \\
\cline { 2 - 3 } & $50000-100000$ & 0.3 \\
\cline { 2 - 3 } & $25000-50000$ & 0.5 \\
\hline \multirow{3}{*}{ Work flexibility } & $15000-25000$ & 0.7 \\
\cline { 2 - 3 } & $<15000$ & 0.9 \\
\cline { 2 - 3 } & high & 0.4 \\
\hline \multirow{3}{*}{ Eco-friendliness } & average & 0.5 \\
\cline { 2 - 3 } & low & 0.6 \\
\cline { 2 - 3 } & not concerned & 0.3 \\
\hline \multirow{3}{*}{ Weather } & medium engagement & 0.5 \\
\hline & high engagement & 0.7 \\
\cline { 2 - 3 } & Good (no rain or snow, and temp $\left.>10^{\circ} \mathrm{C}\right)$ & 0.2 \\
\hline & Average (no rain or snow and temp $\left.0-10^{\circ} \mathrm{C}\right)$ & 0.5 \\
\hline
\end{tabular}

\subsection{Generation of travel alternatives}

For each trip of a traveler, ASIMUT generates a set of travel options, using web services of online travel planners. The attributes that are extracted from the web service, for each travel option include route specification, travel time, cost, $\mathrm{CO}_{2}$ emission, and 
the number of changes. These attributes are later used as input data in the traveler decision-making model (see Section 3.2 for details).

Waking, cycling, and driving travel options refer to the options that use only one of walking, cycle, and car as the mode of transport all the distance from the origin to the destination. Public transport options refer to the travel options that use public transport together with some short walking to and from public transport stops. They might also include transferring between stops. The time and distance of these short walks are taken into account in the simulation. We further complete the set of travel options by adding additional options where we have replaced long walking distances from origin (A) to a station $\left(\mathrm{A}^{\prime}\right)$, and from a station $\left(\mathrm{B}^{\prime}\right)$ to destination $(\mathrm{B})$ by cycling. Long walking is defined as walking distances $(d)$ between 200 meters and 6000 meters. The different travel options from point A to point B are illustrated in Fig. 1.

We use the Google Maps direction API $^{1}$ in order to generate walking, cycling, and driving travel options. The cost for the driving option is calculated based on the travel distance and parking fees if the latter apply. To generate the public transport travel options, web services by the public transport providers in the area are needed. In our case, i.e., the most southern part of Sweden, the public transport travel options are provided by the Skånetrafiken Open $\mathrm{API}^{2}$. It provides cost, travel time, number of changes, $\mathrm{CO}_{2}$ emission, and walking distance of each travel option from point A to point $\mathrm{B}$ in a specified time and date. We have also used an API called "Commute Greener" ${ }^{3}$ in order to calculate the amount of $\mathrm{CO}_{2}$ emission for car users. The output of the APIs is in $\mathrm{XML}^{4}$ or $\mathrm{JSON}^{5}$ schema format. These schemas are parsed in order to extract relevant information, e.g. travel alternatives, travel time, cost, and $\mathrm{CO}_{2}$ emissions of each alternative.

A

\section{A}

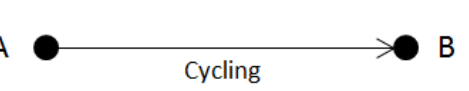

A

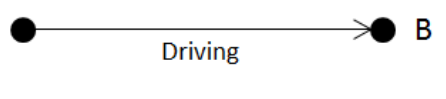

A

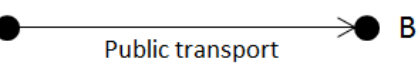

A

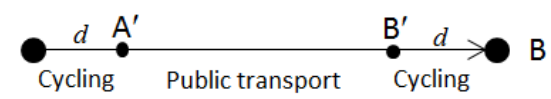

A

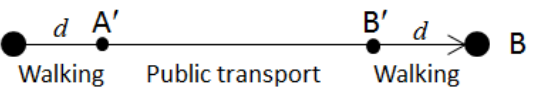

A

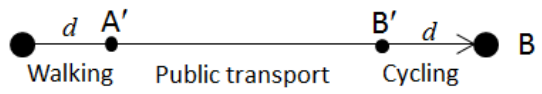

A

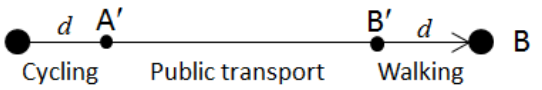

Fig. 1. All considered combinations of transport modes for generating travel options of a trip

When generating the travel alternatives from web services, the characteristics of the traveler are taken into account, i.e., in case the traveler has no access to bike at home, the travel options that include cycling from home will not be generated for that

\footnotetext{
https://developers.google.com/maps/documentation/directions/

http://www.labs.skanetrafiken.se/

$3 \mathrm{http} / / /$ developers.commutegreenerinfo.com/

4 http://www.w3.org/XML/Schema

5 http://json-schema.org/
} 
specific traveler, or if the traveler has no access to car, driving options will not be generated. Furthermore, the source and destination of travel options for a specific traveler, and the departure time of the travel are set according to the traveler's information i.e., work/home address and working hours.

Since it is not possible to obtain detailed weather forecast for more than 14 days ahead, we used historical weather data of the same day as the travel date from the last year provided by the Weather Underground service ${ }^{6}$. This service provides temperature, precipitation, and weather conditions (i.e., rainy or snowy) of the same day for the last year. The sequence of steps performed by the model is illustrated in Fig. 2.

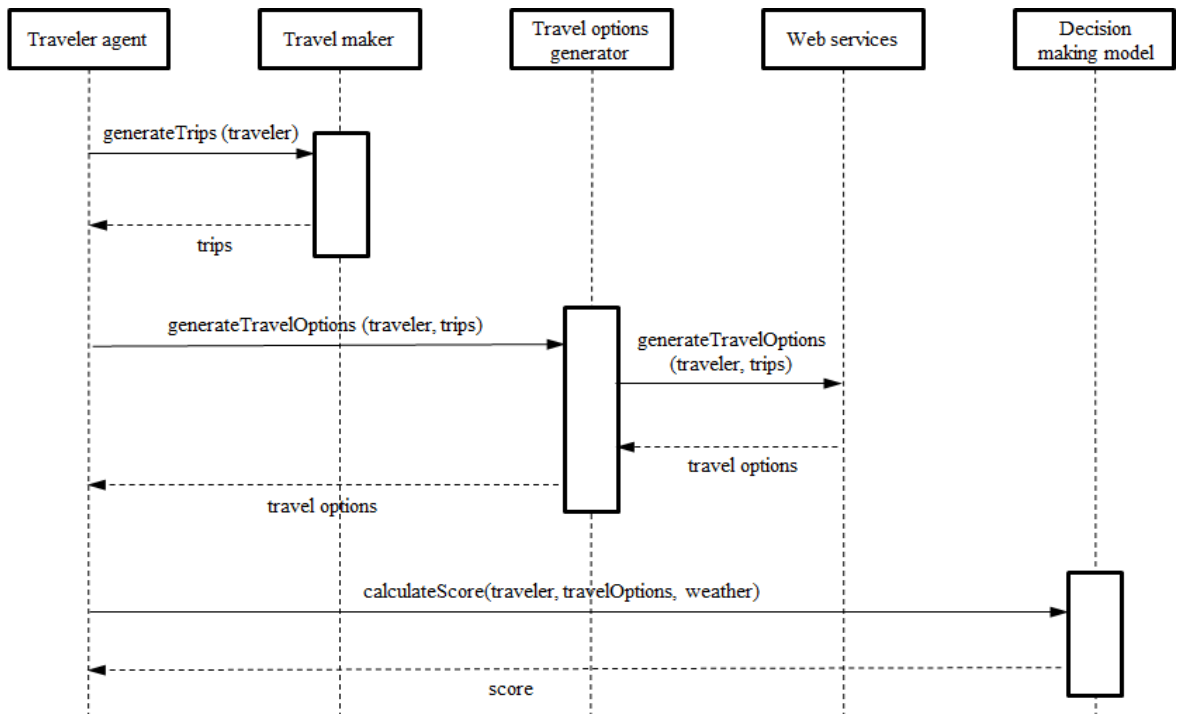

Fig. 2. Sequence diagram of ASIMUT.

\section{Case study}

In this section, we present a small case study that is implemented within a prototype of ASIMUT. In this first basic experiment, we use a small sample population of 16 real travelers from the cities of Malmö and Lund in Sweden, who commute between the cities for work and study. This population sample provides the socio-demographic attributes of the travelers, including their work and home addresses.

For each traveler, we generate two trips for commuting to work and back to home respectively, using the traveler's home and work address and work schedule. Travel alternatives are generated for each trip using web services. A score is calculated for each travel option using our decision-making model.

We study three scenarios; in the first scenario, we simulate the current situation (CS), in the second scenario we examine the effects of reducing the public transport

6 http://www.wunderground.com/ 
fare to half of the price (HP). The third scenario concerns doubling the public transport fare (DP). We investigate how these changes to the public transport fare are expected to affect the choice of travel and the modal share of the travelers using our implemented prototype. We run the simulation for ten randomly generated days with different weather conditions. The diagrams in Fig. 3, Fig. 4 illustrate how changing the public transport fare is expected to affect the modal share, amount of $\mathrm{CO}_{2}$ emission (estimated $\mathrm{CO}_{2}$ footprint per traveler), and travel cost and time for the travelers' commuting during 10 random simulated days. It can be seen from the diagrams that reducing the public transport fare significantly affects the choice of travel and shifts the modal share from private vehicle use to public transport. The walking and cycling share decrease in the DP scenario, which we believe is mostly due to the small walking distances between public transport stations, or also due to the travelers who have combined cycling and public transport. When the travelers switch from public transport to private car, the mentioned walking and cycling links will also disappear. Therefore, we observe a decrease in walking and cycling share in the DP scenario. Furthermore, it can be seen from the Fig. 4 that the amount of $\mathrm{CO}_{2}$ emission is expected to decrease when reducing public transport fare in HP scenario, which can be due to the shift from car use to public transport. Moreover, the selected travel option of the agents cost more when we increase the public transport fare in DP scenario, which can be both because of the increase in public transport fare and the shift to car that is a more expensive mode of transport.

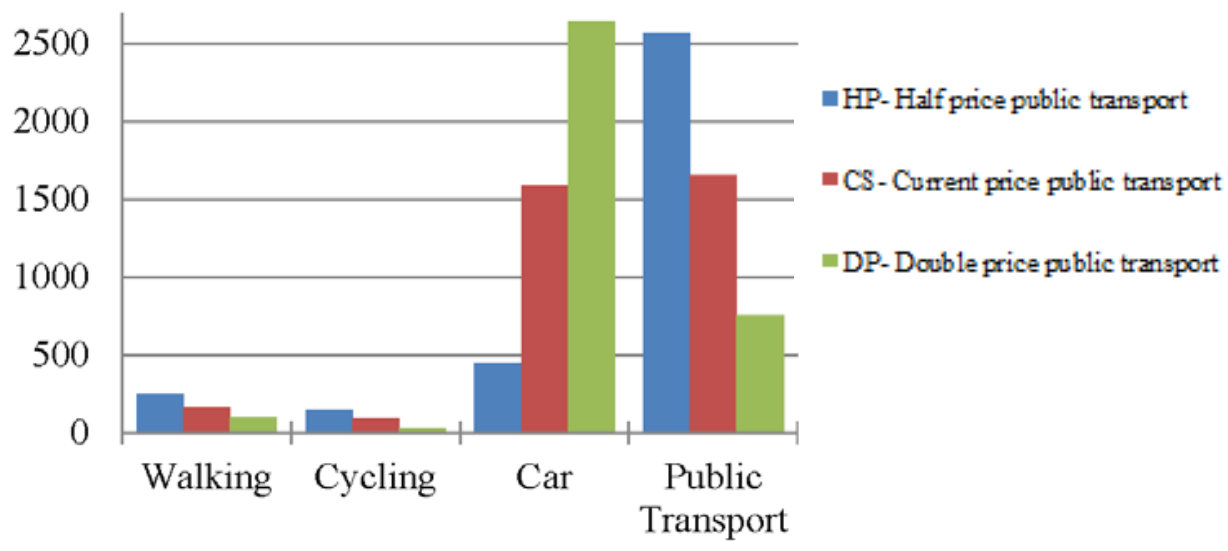

Fig. 3. Modal share $(\mathrm{Km})$ for 10 random days. 

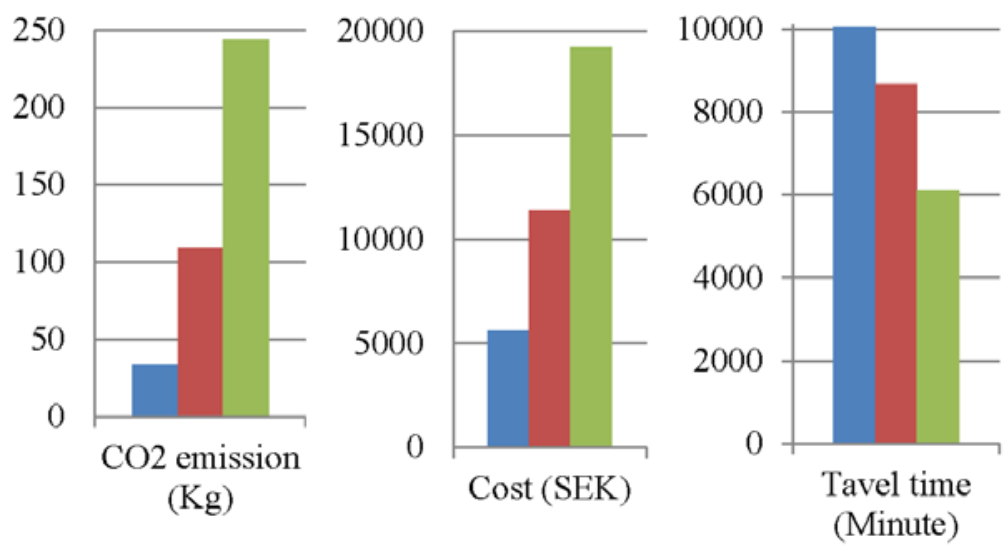

Fig. 4. $\mathrm{CO}_{2}$ emission, cost and time of selected travel options for 10 random days. Blue $=$ half price public transport, red $=$ current price public transport, and green $=$ double price public transport scenario.

\section{$5 \quad$ Concluding remarks}

This paper has presented an innovative multi-agent based simulation model ASIMUT for modeling travel behavior of passengers. The aim is to support policy makers and urban transport planners in estimating the effects of new transport measures, e.g. policies and infrastructure investments. Some of the characteristics of ASIMUT are:

- It uses combinations of transport modes for generating travel alternatives.

- It uses web services of online travel planners to generate travel options.

- It investigates mode, route, and departure time choice of travelers.

- It considers a range of factors influencing the choice of travel in the travel behavior model, i.e., traveler characteristics, contextual data, and social norm.

Using online travel planners enabled us to access real-time network data that to a large extent corresponds to the data that the real travelers are able to access. It also helped reducing the effort and computation required for generating travel alternatives, calculating travel time, cost, and emissions within ASIMUT. It should also be noted that the use of web services as an input data source may have some potential drawbacks. Firstly, the web services might be temporarily down. Secondly, the performance of web services at a given time might be influenced by the load of the service at that time. Although these potential issues can affect the performance of ASIMUT, we did not notice any of these problems during the development and testing. In order to support the scalability of this approach, we currently cache travel options in order to minimize the number of requests. As a future extension of the approach, we will consider the possibility to run our own server.

We have also described the decision-making model and how the travelers choose between generated travel alternatives. We have included convenience factor in 
ASIMUT, which is a combination of walking distance, cycling distance and the number of changes in a travel alternative. The initial results from our case study show the feasibility of our approach in travel behavior modeling.

Future work consists of improving the decision-making model in different ways, such as including more factors (e.g. reliability), and investigating the best way to model the correlation between factors, e.g. how income influence the value of travel time. We will also validate the factors considered in the decision-making model and their influence on the travelers' decision-making. The convenience factor can be further developed to include more factors, such as availability of parking facilities. At this stage, social norms only concern environmental awareness, however, this will be further developed in future versions of ASIMUT. We will also investigate the possibility to consider factors like safety and health, for example avoiding walking through parks during night and choosing to walk or bike instead of car or public transport as a choice for healthier life style. The interaction between travelers will also be considered in the further work, e.g. in the form of car-pooling options. We have also planned to apply synthetic population methods in order to generate large populations of realistic agents. Moreover, we will further test ASIMUT through more complicated scenarios, where the effects of combinations of transport measures are investigated.

Future work also includes analyzing the performance of web services, focusing on how the approach scales with increasing number of simulated travelers. In addition, web services typically behave as black boxes, where the users have little (or no) insight in how the services actually operate. To be able to trust the output generated by a model that is based on externally provided web services, it is therefore critical to take special consideration to the output of the web services when validating the model. Future work also includes analyzing issues related to the use of services that cannot directly be validated.

Acknowledgements. We wish to thank K2 - The Swedish National Knowledge Centre for Public Transport for partially funding this research, and the National ITS Postgraduate.

\section{References}

1. Ajzen, I. (1991). The theory of planned behavior. Organizational Behavior and Human Decision Processes, 50(2), 179-211

2. Alpizar, F., \& Carlsson, F. (2003). Policy implications and analysis of the determinants of travel mode choice: an application of choice experiments to metropolitan Costa Rica. Environment and Development Economics, 8(04), 603-619.

3. An, S., Cui, J. X., \& Li, L. Y. (2011). Agent-based approach to model commuter behaviour's day-to-day dynamics under pre-trip information. IET Intelligent Transport Systems, 5(1), 70-79.

4. Balmer, M., Rieser, M., Meister, K., Charypar, D., Lefebvre, N., Nagel, K., \& Axhausen, K. (2009). MATSim-T: Architecture and simulation times. Multi-agent systems for traffic and transportation engineering, 57-78.

5. De Witte, A., Hollevoet, J., Dobruszkes, F., Hubert, M., \& Macharis, C. (2013). Linking modal choice to motility: A comprehensive review. Transportation Research Part A: Policy and Practice, 49, 329-341. 
6. Gilbert, N., \& Troitzsch, K. (2005). Simulation for the social scientist. Open University Press.

7. Grimaldo, F., Lozano, M., Barber, F., Guerra-Hernández, A. (2012). Towards a model for urban mobility social simulation. Progress in AI, 1(2): 149-156.

8. Hajinasab, B., Davidsson, P., \& Persson, J. A. (2014). A Survey on the Use of Computational Models for Ex Ante Analysis of Urban Transport Policy Instruments. Procedia Computer Science, 32, 348-355.

9. Heinen, E., van Wee, B., \& Maat, K. (2010). Commuting by bicycle: an overview of the literature. Transport reviews, 30(1), 59-96.

10. Hine, J., \& Scott, J. (2000). Seamless, accessible travel: users' views of the public transport journey and interchange. Transport Policy, 7(3), 217-226.

11. Horton, F. E. (1972). Behavioral models in transportation planning. Transportation Engineering Journal of the American Society of Civil Engineers, 98, 411-419.

12. Johansson, M. V., Heldt, T., \& Johansson, P. (2006). The effects of attitudes and personality traits on mode choice. Transportation Research Part A: Policy and Practice, 40(6), 507-525.

13. Lave, SC. A. (1969). A behavioral approach to modal split forecasting. Transportation Research, 3(4), 463-480.

14. McDonnell, S., \& Zellner, M. (2011). Exploring the effectiveness of bus rapid transit a prototype agent-based model of commuting behaviour. Transport Policy, 18(6), 825-835.

15. Nitta, Y., \& Do, G. (1998). Transportation mode-change model to special bus incorporating generalized time. In 8th International Conference on Transport and Mobility for Elderly and Disabled People.

16. Ramstedt, L. (2008). Transport policy analysis using multi-agent-based simulation, Doctoral Dissertation Series No. 2008:09, Blekinge Institute of Technology, Sweden.

17. Takama, T., \& Preston, J. (2008). Forecasting the effects of road user charge by stochastic agent-based modelling. Transportation Research Part A: Policy and Practice, 42,738-749.

18. Teodorovic, D. (2003). Transport modeling by multi-agent systems: a swarm intelligence approach. Transportation Planning and Technology, 26(4), 289-312.

19. Triantaphyllou, E. (2000). Multi-criteria decision making methods a comparative study. Springer.

20. Walle, S. V., \& Steenberghen, T. (2006). Space and time related determinants of public transport use in trip chains. Transportation Research Part A: Policy and Practice, 40(2), 151-162. 



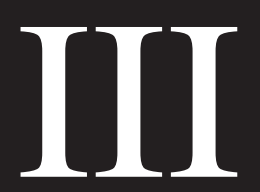



Paper 3: The Fourth Wave of Digitalization and Public Transport: Opportunities and Challenges

\title{
Authors:
}

Paul Davidsson, Banafsheh Hajinasab, Johan Holmgren, Åse Jevinger and Jan A. Persson

\section{Published:}

Sustainability Journal 8, no. 12 (2016): 1248.

\begin{abstract}
We investigate the opportunities and challenges of the forth wave of digitalization, also referred to as the Internet of Things (IoT), with respect to public transport and how it can support sustainable development of society. Environmental, economical, and social perspectives are considered through analysis of the existing literature and explorative studies. We conclude that there are great opportunities for both transport operators and planners, as well as for the travelers. We describe and analyze a number of concrete opportunities for each of these actors. However, in order to realize these opportunities, there are also a number of challenges that needs to be addressed. There are both technical challenges, such as data collection issues, interoperability, scalability and information security, and non-technical challenges such as business models, usability, privacy issues, and deployment.
\end{abstract}


Article

\title{
The Fourth Wave of Digitalization and Public Transport: Opportunities and Challenges
}

\author{
Paul Davidsson *, Banafsheh Hajinasab, Johan Holmgren, Åse Jevinger and Jan A. Persson \\ Internet of Things and People Research Center, K2-The Swedish Knowledge Centre for Public Transport, \\ Department of Computer Science, Malmö University, Malmö 20506, Sweden; \\ banafsheh.hajinasab@mah.se (B.H.); johan.holmgren@mah.se (J.H.); ase.jevinger@mah.se (Å.J.); \\ jan.a.persson@mah.se (J.A.P.) \\ * Correspondence: paul.davidsson@mah.se; Tel.: +46-40-665-8618
}

Academic Editors: Wijnand Veeneman and Eefje Cuppen

Received: 7 September 2016; Accepted: 24 November 2016; Published: 30 November 2016

\begin{abstract}
We investigate the opportunities and challenges of the forth wave of digitalization, also referred to as the Internet of Things (IoT), with respect to public transport and how it can support sustainable development of society. Environmental, economical, and social perspectives are considered through analysis of the existing literature and explorative studies. We conclude that there are great opportunities for both transport operators and planners, as well as for the travelers. We describe and analyze a number of concrete opportunities for each of these actors. However, in order to realize these opportunities, there are also a number of challenges that needs to be addressed. There are both technical challenges, such as data collection issues, interoperability, scalability and information security, and non-technical challenges such as business models, usability, privacy issues, and deployment.
\end{abstract}

Keywords: public transport; Internet of Things; digitalization; opportunities; challenges

\section{Introduction}

We are currently in what can be seen as the fourth wave of digitalization. The first wave came in the $80 \mathrm{~s}$ and consisted in the introduction of computers in many parts of society. In the $90 \mathrm{~s}$, the second wave in the form of the Internet made it easy to access and share information. The third wave is the mobile Internet that enables this regardless of where you are. With the fourth wave of digitization, it is not just people who use the Internet to access and share information, but also different types of entities, such as vehicles, appliances, and machinery. All parts of our society will be affected by this fourth wave, commonly referred to as the Internet of Things (IoT) [1].

Strongly related to IoT and the fourth wave of digitalization is the concept of ubiquitous computing first articulated by Mark Weiser at Xerox Parc more than 25 years ago [2]. His vision about calm technology requires computers to be imbedded into the environment in such a way that we use them without even thinking about their existence. This notion of computing enables users to use computers anywhere and anytime. In order to realize the envisioned ubiquitous computing by Mark Weiser, much research and development has been carried out on embedding computers into physical objects and to make them smarter (physical computing), connecting these smart objects to the Internet (Internet of Things), integrating computers with textile and clothes (wearable computing), distributing computation and user interface among several computing devices, etc.

Together with some other recent technological and methodological developments in information technology, such as open data, big data, sensor technology, and crowdsourcing, we argue that IoT can be seen as a powerful enabler of sustainable development in the context of public transport. In particular, the collection of different types of data can be made much easier, more accurate, and 
in real-time, through the use of IoT. Examples are data related to one or more of the following types of entities:

- Vehicles: location, occupancy level, vehicle status, presence of on-board staff, etc.

- Travelers: time and location of entering and leaving vehicles, individual preferences and final destination, ticketing data, etc.

- Infrastructure: the status of transport links, e.g., congestion, the number of people in a certain location, e.g., at a bus stop, etc.

The data can be either provided automatically through different types of sensors and machines, or via the travelers themselves through different types of smartphone applications. Such applications could either collect data automatically (e.g., position and velocity) or manually (e.g., travelers' preferences and their final destinations). The data can be gathered via dedicated travelers' information applications or via general applications such as Facebook. The availability of the abovementioned data, as well as other types of relevant data is increasing rapidly, and may be further accelerated by the voluntary provisioning of data by individual travelers. The efficient management and use of this large amount of data, often referred to as "big data" [3], are key issues for the ability to achieve the full potential of IoT, e.g., as an enable of more sustainable public transport. As the data typically is collected in real-time, it has the potential to impact real-time decisions by transport operators and travelers. However, the collected data is also highly relevant for the strategic planning of transports and the development of the transport system. In addition to just simply generate more data, IoT also has the potential to improve data quality, e.g., by fusing data from multiple sensors.

Moreover, IoT solutions can help the travelers to interact with the public transport system in a more efficient way through, e.g., supporting ticket purchasing and enabling the use of personalized and context-aware information services, such as traveler planners that make use of information such as, on which public transport vehicle the traveler is currently riding.

The potential in digitalization does not only concern collecting data and providing information about services. IoT is also a key ingredient for enabling remote control of vehicles and infrastructure, and even introducing autonomous action, e.g., autonomous vehicles that probably will be a vital part of the future public transport system.

In the context of public transport, digitalization can support the development of a more sustainable society in many ways, for example, the following:

- Environmental sustainability:

a. By optimizing routes, time-tables, style of driving, vehicle sizes, etc., the direct emissions from the public transport system can be reduced.

b. By increasing the attractiveness and use of public transport, it is possible to reduce the in-direct emissions (i.e., the emissions from the non-public transport system) and improving land use (e.g., reducing the space dedicated for car parking).

- Social sustainability:

c. By increasing accessibility to public transport for vulnerable groups (e.g., elderly, disabled, and ethnic minorities), it is possible to improve social equity in public transport.

d. By increasing the coverage of public transport services, equity and sustainable living is supported. In addition, by increasing the coverage of public transport, the access to different activities will increase, and therefore, the feeling of social inclusion and life satisfaction might increase.

e. By increasing public transport safety, personal safety can be improved. 
- Economical sustainability:

f. By optimizing the use of resources in public transport, money can be saved by the public transport providers.

g. By minimizing the travel time, the travelers can do more productive things than traveling and waiting for transport services.

In this article, we will investigate the opportunities as well as the challenges of the fourth wave of digitalization with respect to public transport and how the opportunities may contribute towards a sustainable society. There exist some previous analyses of the digitalization of public transport, such as $[4,5]$. Our contribution compared to those is that we take the recent developments in IoT and mobile Internet, as well as the sustainability perspective into account.

\section{Opportunities}

As mentioned in the introduction, the fourth wave of digitalization makes different types of data collection easier, more accurate, and in real-time. In addition, it provides a means for new types of remote control and automation. These developments enable new opportunities for nearly all aspects of public transport, including:

- Operations: opportunities from the transport operators' perspective.

- Planning: opportunities from the strategic transport planners' perspective.

- Travelling: opportunities from the travelers' perspective.

In this section, we will explore the opportunities from these three perspectives.

\subsection{Operations}

We refer to operations as the management of the resources used in public transport. In this subsection, we focus on the real-time aspects of operations, i.e., the actions and decisions that are not considered transport planning (see Section 2.2). Obviously, the data provided by IoT systems give new opportunities and can be used to support better resource utilization and better services in general.

In public transport systems, most transport services are timetabled, and an important opportunity is to use the real-time information enabled by IoT in order to deal with deviations from the original plan, i.e., in order to handle disturbances. Traditionally, the focus of action in such cases is to re-plan in order to restore the transport system according to the original plan and, e.g., get the stranded travelers to their destinations [6]. See [7] for an overview of the existing literature and discussions on this topic. In general, real-time data can improve the re-planning decisions through enabling the planners to base their decisions on more data and/or more accurate data. Moreover, IoT makes it possible to better communicate the taken actions to travelers and operators, e.g., through providing up-to-date information to the on-board staff on a train. However, re-planning actions and decisions might influence the sustainability both positively and negatively, which is why access to accurate, up-to-date information is important. For example, public transport may in fact increase emissions compared to using car travel, for example, if there are few passengers in a bus (in case of low fill rate).

Examples of opportunities:

- Improved management of operations based on knowledge about vehicles and travelers currently on, or planning to be on, a transport route, e.g., through ticketing data, sensor data, and data from travelers [8,9].

Manage unplanned situations. Improved management may be of particular interest when unplanned situations occur, e.g., when a train departure is cancelled and needs to be replaced by bus, or when a vehicle is delayed. In such cases, decisions might need to 
be taken on how many replacement buses (or taxis) that should be used. Decisions might also need to be taken regarding re-allocation or re-scheduling of other vehicles. For example, decisions regarding whether a bus or train should wait for another delayed vehicle could be made with better information at hand, e.g., on how long is the expected waiting time and how many travelers are expected to board the waiting bus or train. IoT can in different ways provide information relevant for such decisions, which is useful (or necessary) for efficient traffic management, see for instance [10,11]. Examples of relevant information potentially provided by IoT are how many travelers are currently on a public transport vehicle and what are their intended destinations. See also [12] for early examples on methods on how to decide whether a train or a bus should wait or not for another vehicle. In terms of sustainability, unnecessary resource utilization may, potentially, be avoided (e.g., through minimizing the number of replacement vehicles). Hence, there is also a potential to reduce the direct emissions from public transport. In addition, we expect the passengers' waiting time, in the general case, to decrease due to improved disturbance management. In addition, the indirect emissions might eventually decrease as a consequence of the possibility to offer more robust, hence more attractive, public transport services that the travelers might prefer over the private car alternative.

Demand Responsive Transport (DRT). In DRT, passengers share a vehicle, for instance a small bus, which picks up and drops off the passengers at passenger-specified locations and times [13]. Initially, DRT was designed for disabled or elderly people (see [14,15]). However, it is now seen as an interesting solution for increased access and flexibility in public transport, for the whole community $[16,17]$. In particular, it enables the use of more dynamic and flexible bus routes based on the passengers' preferences. For instance, a bus may only visit the bus stops where the travelers on the bus want to exit and where there are people actually waiting. Hence, DRT may provide improved sustainability in terms of increased accessibility and improved coverage. In the long run, DRT might also lead to reduced indirect emissions as a direct consequence of enabling more attractive, passenger adapted, public transport alternatives. Depending on the implementation, there is also a potential for decreased direct emissions of public transport, e.g., if buses are allowed to take shortcuts in order to avoid stops where no passengers are waiting, or if it is possible to replace large buses with smaller, more fuel-efficient, vehicles when demand is expected to be low. Depending on the implementation, and on the passengers behavior, it is also possible to achieve reduced travel times for the passengers by advising them to walk to nearby bus stops to minimize their waiting time or to catch a fast transport service.

Maintenance-wear. Through the use of sensor data about the status of vehicles, it is possible to make more accurate decisions on when vehicles need maintenance, which may result in fewer scheduled and unscheduled stops for maintenance and repair, and potentially less maintenance time in total. See for instance [18] for an early sensor-based approach for vehicle maintenance. Improved sustainability may be achieved mainly in terms of improved resource utilization. Sustainability aspects that might be supported indirectly are safety and travel time. Safety might be improved through using vehicles in better condition (avoiding malfunctioning brakes, worn out tires, etc.) and by reducing the risk of dangerous evacuations (due to unscheduled stops) of the passengers, e.g., at roads with high traffic density. We expect unnecessary waiting time to be avoided due to fewer unscheduled stops. In addition, vehicles in need for maintenance, e.g., trains with damaged wheels and pantograph strips, might cause infrastructure damage that may cause delays and additional waiting time, which might be avoided, for other vehicles sharing the same infrastructure.

Maintenance-damage. Related to maintenance due to wear and tear, there is also a possibility to use IoT to collect information about damage on the vehicles and the 
infrastructure used in public transport. For example, travelers can submit reports on malfunctions, e.g., broken seats or broken seat belts, possibly together with context data collected from the vehicles. If the traveler receives immediate response, the traveler satisfaction may increase. A potential is to use crowdsourcing that enables the operator (in addition to other travelers) to take quick actions, such as, repairing damages, see for instance [19]. This type of systems might contribute to improved sustainability through reduced indirect emissions by enabling more attractive public transport services and through increased safety by achieving quicker identification and repair of safety critical equipment.

- Self-driving vehicles. Self-driving vehicles, which are enabled by IoT technology, may influence the transport system significantly [20,21]. Replacing drivers will reduce the cost and most probably also improve the safety of transport. Improved driving pattern, e.g., eco-driving, can be highly prioritized in the self-driving vehicles and hence lead to reduced emissions. An interesting opportunity that appears with self-driving cars is that they can be time-shared in a quite easy way, since the re-allocation to the next user can be achieved without involving human drivers. For instance, a user may rent a self-driving car for the weekends and the very same car may be used for public transport (i.e., as taxi) during the weekdays, and the user does not need to worry about the reallocation between the different purposes every week. In summary, self-driving cars may contribute to improved sustainability in terms of improved resource utilization and reduced emissions, and potentially improved safety depending on the implementation.

- $\quad$ Transport related services. In addition to enabling new services and improved decision making for the traditional public transport operators, IoT might create opportunities for transport-related services. Reduced travel time can be achieved, for instance, by providing information, for bike renters and taxi companies, that supports the efficient allocation of non-occupied bikes and taxis. Rental bikes can support more attractive public transport by, for example, enabling travelers to travel to and from bus stops and terminals using rented bikes. It is also possible to create services that help travelers find available taxis and bikes. For example, a successful approach with replacement taxis is applied in Munich [22]. Altogether, the abovementioned improvements may directly lead to reduced travel time and more attractive public services, hence supporting the sustainability aspect of reduced indirect emissions.

\subsection{Planning}

One of the major challenges in public transport is to plan the resources in such a way that they are best utilized for providing the best possible services to the travelers and for minimizing the negative implications of transport, including $\mathrm{CO}_{2}$ emissions and noise. The planning issues related to public transport includes, but are not limited to, choosing bus and train routes (or lines), determining frequencies and timetables of the vehicles that travel along the routes, and localization of stops. The planning issues might also concern decisions on implementing new technologies, such as ITS services in public transport. The difficulty of planning is often due to the lack of information feedback from the transport operations, i.e., the transport planners do not always have access to enough information, e.g., about individual trips in order to plan the transport resources in the best possible way. By increasing the use of IoT-based services in transport operations, there is a unique opportunity for transport planners to collect huge amounts of data ranging from travel behavior to infrastructure usage, which can be used to achieve improved planning of the public transport resources, including vehicles and infrastructure.

Transport modeling and simulation is one of the most common approaches for supporting the decision making in transport planning through, e.g., enabling to predict the impacts of new transport policies and infrastructure investments. Based on the questions that a simulation model needs to answer, different types of data, ranging from socio-demographic attributes of people to the transport network data, needs to be provided to the model. In the area of transport system modeling and 
simulation, there is an opportunity to access data that is automatically collected using, e.g., IoT devices, and to outsource some of the computations that traditionally are carried out within the simulation model, using open web services like Google Maps.

Examples of opportunities:

- An obvious opportunity for the transport planners is to retrieve new types of data, or more accurate data, through the use of IoT systems, smartphones, and crowdsourcing systems. The retrieved information could be used to support tasks such as, determining optimal bus routes and timetables, in a better way than is typically possible today.

Collection of traveler data. Smartphone applications can be used to extract movement patterns of their owners and to identify, for example, the chosen transport mode, i.e., car, bus, bike, walking, etc. $[23,24]$. Examples of other types of data that may be captured in similar ways are which transport services individual travelers use, and when they enter and leave services. In terms of improved sustainability, this type of data may be used to enable better utilization of transport resources, for example, through supporting the design of more efficient routes, which is expected to result in reduced emissions from the vehicles used in public transport, i.e., our direct emissions sustainability aspect. Other sustainability aspects that might be supported, depending on which aspects are considered in the route design process are accessibility, coverage, and travel time. As a consequence of improved routes, there is also a potential to support the indirect emissions aspect. However, we expect this to be highly dependent on whether the improved public transport routes, enabled through the use of travel data, appeals to the travelers that traditionally prefer the private car alternative.

- Collection of vehicle data. On the vehicle level, the use of IoT makes it is possible to automatically collect detailed travel data on, e.g. when passengers enter and leave vehicles, occupancy rates (e.g., by measuring the number of available seats through sensors), and robustness (frequent delays). Realistic and high quality data of the abovementioned types might be valuable for travel planners in order to improve the planning of the resources used in public transport. Improved resource utilization and reduced direct emissions can be achieved through using information about the current use of vehicles, e.g., by using mini-buses at times where few travelers are expected. However, we believe there is a risk that public transport becomes less attractive if vehicle data is used to support decisions on choosing "too" small buses. On the other hand, there is a potential to reduce indirect emissions through designing more attractive services, e.g., by using extra buses at times where the buses are typically crowded.

Collection of traffic data. Online traffic monitoring [25,26], e.g., measuring congestion levels through crowdsourcing and road-side cameras, might give input that can be used to reduce the direct emissions and travel times of public transport, e.g., through supporting the generation of improved routes, where congested roads might be avoided. Traffic data can also make public transport more attractive by supporting the design of services along routes where driving is often slow due to heavy congestion, perhaps utilizing bus lanes, hence indirectly contributing to reduced indirect emissions. In addition, there is a potential to use traffic data to improve personal security, our safety sustainability aspect, for example, through supporting decisions on building safer bus stops along roads that are dangerous due to, e.g., high congestion or high driving speeds.

Collection of air quality data. Air pollution monitoring $[27,28]$ provide information that might be used to identify the need for more environmentally friendly transport services, for example, using public transport vehicles that produce less emissions. It may also support decisions regarding the introduction of public transport services that travelers might prefer over private car transport in areas with bad air quality. Through making the 
decision makers aware about air quality problems in particular areas, this type of data can be used to support sustainability mainly in terms of direct emissions.

- Collection of transfer point data. Detailed information about transfer points between services can be used to support optimization of timetables so that travelers in a better way than today can utilize routes involving multiple public transport services. An example of relevant transfer point data is data about people movements captured by different types of sensors. Transport services that are connected in a better way may reduce the overall travel time, hence improving the economical sustainability for the travelers. As a consequence of improved timetables, transfer point data can also support sustainability by reducing the emissions from non-public transport through enabling more attractive public transport services. In addition, information about transfer points might be used to support decisions on adapting transfer points to elderly and weak traveler groups, hence improving accessibility.

- Use online services for modeling. Using online services to access the data collected by IoT devices and smartphones (including crowdsourcing systems) as well as of different types of processed data, there is an opportunity to construct analysis and problem solving models that work with data in a new way [29]. For example, transport simulation models for analysis of public transport might use online services in order to access, e.g., weather data and current travel times, collected by different types of IoT sensors. A special type of online service, which is relevant to mention here is travel planner systems. Travel planner systems, mainly smartphone applications, are becoming more widespread, and we find it reasonable to assume that the choices of travelers' to some extent is changing based on suggestions provided by such systems. Therefore, we believe that the use of travel planners to estimate people's travel behavior can increase the accuracy of the travel behavior modeling in simulation systems. In terms of planning, we argue that the use of online services has the potential to influence all types sustainability aspects discussed above, however, indirectly through the use of the output from various types of models.

\subsection{Travelling}

A common problem of today is that the travelers often are unable to make informed choices due to lack of information, or due to lack of up-to-date information. Efficient approaches need to be developed in order to provide travelers with the correct information in their current situations. Such information may concern, for instance, what travel options are actually available in a given situation, where tickets can be bought, and what support a specific transport service offers to elderly or disabled persons. By providing enhanced information and services through IoT, the behavior of the travelers may change; with more information at hand, people are able to make more informed travel decisions, and old travel habits following the same patterns can be broken. In particular, enhanced information and services have the potential to increase the attractiveness of public transport, which may encourage more travelers to choose public transport over the car and in that way contribute to improved sustainability.

IoT technology can be used in different ways to collect data for traveler services. For instance, delay information can be obtained from recognition or positioning sensors on-board vehicles and at stops [5], information about the travelers' actual destinations (used to provide efficient transport alternatives) can be collected from smart card tickets [30], and micro-navigation information can be attained using IoT-based recognition systems that are located on-board vehicles and across stations [31]. In some situations, data similar to those collected already exist in other types of systems. However, IoT provides an opportunity to increase the level of detail and the accuracy of the data. For instance, the positioning system available in smart phones can typically not be used for detailed indoor navigation guidance, both because the accuracy of the positioning data may be too low and because indoor facilities are not taken into account. Instead, context-aware information systems provided by IoT technology might be used to provide accurate indoor navigation guidance. 
Examples of opportunities:

- Real-time delay information. Based on information about delayed or cancelled transport services, and about the context, the traveler is able to make informed decisions on which transport service to select during a disturbance. A service for supporting such decisions can be made personalized though the use of information about the current location of the traveler, e.g., on which bus or terminal the traveler currently is located [31]. Thereby, alternative travel routes can be suggested to the traveler. Hence, the real-time delay information supports the travel time sustainability aspect by helping the travelers to reach their destinations with minimum delay during disturbance. Eventually, improved real-time delay information might also support the reduction of indirect emissions by offering transport services that are more predictable, and therefore more attractive. Moreover, travelers with specific needs, e.g., elderly or disabled, can receive customized real-time information about transport alternatives with high accessibility. Real-time information about delays can be provided using IoT technology, e.g., by devices at each bus stop that recognize if the bus has stopped there [32].

- Co-traveler information. Information about which passengers are travelling on the same vehicle can be provided using IoT, e.g., through the use of beacons. This information can be used for connecting people and, together with information about the passengers' destinations, enable shared taxis both during disturbance and to reach the final destination in case this is not possible using bus or train. In terms of sustainability, improved co-traveler information, enabled through the use of IoT is expected to contribute to improved resource utilization and indirectly reduced travel time. We make the assumption that by sharing taxis, resources are indirectly saved and emissions are indirectly reduced in the transport system. We also expect that the indirect emissions will decrease as a consequence of enabling a more attractive service, where people can connect without too much effort. Moreover, this type of service might be of particular value during disturbances.

- Real-time vehicle information. Real-time vehicle information concerns the possibility to make informed decisions on which public transport service to select, based on information about the status and characteristics of the vehicles used in public transport. Firstly, information about the number of passengers on-board a transport vehicle can be utilized by travelers to identify overcrowded vehicles [33]. For instance, they may choose to travel by bicycle instead of waiting for an overcrowded bus. Secondly, with real-time information about the characteristics and status of specific transport vehicles, people with special needs, such as elderly and disabled, can be informed about relevant transport possibilities. This type of information can be directed both to on-board passengers and to people waiting for transport, through the use of IoT. The information can be used for, e.g., informing a traveler of whether a specific bus has support and room for wheelchairs or baby strollers, or whether bicycles are allowed on a specific train waiting on a track. Obviously this type of information has an impact on the accessibility but also on the attractiveness of using public transports. Further it may enable travelers to avoid choosing a vehicle, where there is no available space, and thereby being able to reach their final destinations in less time than without this information.

- Delay compensation. Price compensation for delays due to disturbances can more easily be provided if the travelers are able to prove their presence on a delayed vehicle. This can be enabled by context-aware data provided by IoT. We expect that delay compensation will contribute indirectly (i.e., eventually) to reduced indirect emissions, as the possibility to get compensation might be a factor to choose public transport despite the, often high, risk for delays.

- Interchange guidance. Context-aware provision of information during interchanges can help guiding the traveler, for instance in order to reach the correct train platform or the ticket office, and to help calculating the time required for getting there [32,34]. It is also possible to take into account personal disability constraints, e.g., need for wheelchair. Hence, interchange guidance 
information may affect the accessibility sustainability aspect, and indirectly the travel time aspect if the times for (planned) interchange are reduced as consequence.

- Ticket-buying support. Ticket-buying support involves the ticketing and payment systems with which the traveler may interact. IoT technology can be used to provide positioning information in order to facilitate ticket purchasing. Furthermore, the validity of tickets can automatically be prolonged during disturbances. Easy and efficient ticketing systems influence the attractiveness of the public transport system. Further, it may reduce the time needed for purchasing tickets and thereby contributing to reduced the travel time if implemented correctly, and it may make it easier for travelers with special needs, e.g., children and intellectually disabled, to use public transport.

- Support during travel. Support during travel makes it possible for travelers to make sure that they are acting as planned, e.g., to get confirmation of that they have boarded the correct vehicle, that their tickets are valid, and getting information about when to leave the vehicle, by use of IoT technology [31,35]. Such support can make the public transport system more attractive and potentially also more accessible to larger groups of people (e.g., disabled persons). In addition, by avoiding getting off at the wrong location, the travel times may indirectly be reduced.

- Enriched travel experience. By providing different types of data enriching the travelling experience, public transport can be made more attractive. This may include providing contextual information about the destination or the current surroundings, or providing vehicle data that help travelers to keep track of the environmental load caused by their travel and possibly comparing this load to the load caused by the corresponding car travel.

\subsection{Discussion}

In Table 1, we summarize how the opportunities discussed in Sections 2.1-2.3 support the sustainability aspects presented in Section 1. In the table, " $\mathrm{D}$ " denotes expected direct influence, " $\mathrm{I}$ " expected indirect influence, typically not happening immediately and typically being a consequence of other effects, and parentheses indicate potential influence that is highly depending on the implementation. It should be emphasized that for the planning opportunities, we did not consider the time aspect when categorizing an effect as direct or indirect; we only considered whether the effect is expected as a consequence of other effects (indirect) or not (direct).

Table 1. Summary of how the identified opportunities supports the sustainability aspects.

\begin{tabular}{|c|c|c|c|c|c|c|c|}
\hline Opportunity/Sustainability Aspect & $\begin{array}{c}\text { Direct } \\
\text { Emissions }\end{array}$ & $\begin{array}{c}\text { Indirect } \\
\text { Emissions }\end{array}$ & Accessibility & Coverage & Safety & Resources & $\begin{array}{c}\text { Travel } \\
\text { Time }\end{array}$ \\
\hline Operations: manage unplanned situations & (D) & I & & & & (D) & $\mathrm{D}$ \\
\hline Operations: demand-responsive transport & (D) & $\mathrm{D}$ & (D) & $\mathrm{D}$ & & & (D) \\
\hline Operations: maintenance-wear & & & & & I & $\mathrm{D}$ & I \\
\hline Operations: maintenance-damage & & $\mathrm{D}$ & & & $\mathrm{D}$ & & \\
\hline Operations: self-driving vehicles & $\mathrm{D}$ & & & & (D) & $\mathrm{D}$ & \\
\hline Operations: transport related services & & $\mathrm{D}$ & & & & & $\mathrm{D}$ \\
\hline Planning: collection of traveler data & $\mathrm{D}$ & (I) & (D) & (D) & & $\mathrm{D}$ & (D) \\
\hline Planning: collection of vehicle data & $\mathrm{D}$ & (I) & & & & $\mathrm{D}$ & \\
\hline Planning: collection of traffic data & $\mathrm{D}$ & I & & & (D) & & $\mathrm{D}$ \\
\hline Planning: collection of air quality data & I & I & & & & & \\
\hline Planning: collection of transfer point data & & (D) & $\mathrm{D}$ & & & & $\mathrm{D}$ \\
\hline Planning: use online services for modeling & (I) & (I) & (I) & (I) & (I) & (I) & (I) \\
\hline Travelling: real-time delay information & & I & (D) & & & & $\mathrm{D}$ \\
\hline Travelling: co-traveler information & (I) & I & & & & $\mathrm{D}$ & I \\
\hline Travelling: real-time vehicle information & & (I) & $\mathrm{D}$ & & & & (D) \\
\hline Travelling: delay compensation & & I & & & & & \\
\hline Travelling: interchange guidance & & I & $\mathrm{D}$ & & & & I \\
\hline Travelling: ticket-buying support & & I & $\mathrm{D}$ & & & & (I) \\
\hline Travelling: support during travel & & I & (D) & & & & (I) \\
\hline Travelling: enriched travel experience & & I & & & & & \\
\hline
\end{tabular}

From our analysis of the identified opportunities, we see that the fourth wave of digitalization has a large potential to support sustainable development of public transport in a wide range of ways. 
Further analysis is needed in order to assess the relative size of the influence on sustainability for the different opportunities. However, our analysis suggests that demand-responsive transport and collection of traveler data would have the most significant direct impact on several sustainability aspects. Further, we provide guidance of what opportunities to potentially implement in order to support a specific sustainability aspect. For instance, we can see that there are rather few opportunities that we expect to support coverage, whereas most opportunities support the indirect emissions category, i.e., through increasing the usage of public transport. In addition, by assessing the type of influence, e.g., direct, indirect and the dependency on implementation, we argue that careful analysis of alternatives of implementation is needed for a number of opportunities that we assess as highly dependent. We can also argue that for the cases of indirect influence, additional analysis is motivated since the uncertainty is increased in case of dependency on other effects.

\section{Challenges}

In this section, we discuss some important challenges related to the use of the fourth wave of digitalization (i.e., IoT) within public transport as a successful enabler for a sustainable society (see a discussion on opportunities in Section 2). We discuss the challenges in relation to the following categories: business models, privacy and integrity issues, security, interoperability, scalability, usability, data collection, and deployment.

Obviously, there exist technologies that partially, or at least in some situations, help overcome the identified challenges; some examples of useful technologies are given below. However, it cannot be claimed that any existing solution completely eliminates any of the identified challenges in all possible situations, in particular, since the involved actors' knowledge on existing technologies may complicate their application in order to overcome the discussed challenges.

In order to address the challenges listed below, one should review relevant previous work, especially in the area of Intelligent Transport Systems (ITS). In particular, a number of general ITS architectures, which face different challenges and technological developments, have been proposed. These architectures are on a relatively high level of abstraction, meaning that they are technology-independent specifications on components and communications [36]. Thus, some of the interoperability challenges discussed below may be solved by using one of these ITS architecture. However, there is no single "standard" ITS architecture; different countries and regions have developed their own ITS architectures in order to fit their needs [37]. This lack of a single standard ITS architecture must be handled to develop global solutions.

For the other challenges identified in this paper, the high-level architectures typically do not provide any solutions. However, Dimitrakopoulos and Demestichas [38] describe an example of how to concretize an ITS architecture using the concept of Cognitive Transport Network. The system is based on cognitive and wireless sensor networks (WSNs), and it provides directives to both drivers and infrastructure elements, e.g., traffic lights, with the aim of improving transport efficiency and safety. In particular, it involves a transportation management system with cognitive learning capabilities, which use the data from the WSN to create knowledge and experience about the current and past situations in the transport network. Based on this information, any new decisions taken, including future planning, are improved in terms of performance and reliability [38]. The study is focused on car drivers; however, parallels can be drawn to IoT-based public transport. By using sensor data, in combination with cognitive transport management systems in an IoT-based environment, both planning and operations in public transport can be improved.

In this paper we will not further address technical solutions, since we focus on identifying opportunities and challenges rather than on how to build systems.

\subsection{Business Models}

In order to achieve the full benefits of the fourth wave of digitalization, involvement is typically required by a multitude of actors in addition to the public transport operator, including 
telecommunication operators, sensor data providers, data storage providers, end-user service providers, and the travelers themselves, but also different public authorities. Large investments may be needed in order to build and maintain the necessary infrastructure, the collected data needs to be managed and stored, services and data APIs needs to be provided, etc.

Obviously, the building and maintenance of IoT systems are associated with significant costs for the involved actors; costs that need to be covered for in a reasonable way, in particular since many types of actors are needed in order to enable an efficient IoT system. A major challenge is the need for effective business models, which regulates how the revenues should be distributed in order to cover for the costs of the involved actors. In particular, business models need to regulate the distribution of costs and revenues for sharing and using the collected data and the generated information, and for providing services for end users, companies and other organizations. It is important that there are business opportunities for the companies who want to provide the necessary services but are out of the scope of the transport operators and public authorities; they need to get a return of their investments.

It should be further emphasized that it appears to be easier to get paid the closer to the customer/user one is in the information chain. In the public transport area there are some open data sources, as well as platforms for services (cf. www.trafiklab.se). However, a major challenge concerns how to get paid for commercially gathering of data. Bulger et al. provide a thorough discussion on business models concerning large amounts of data, i.e., "big data" [39].

Since public transport operations are typically under the tight regulation and procurement from governmental agencies, it may be enforced that some data needs to be supplied by the transport operators. However, challenges of, for instance continuity and quality is often an issue and initiatives of other pure commercial and third party actors typically need business models.

\subsection{Privacy and Integrity Issues}

Privacy is about not sharing data about organizations and individuals, whereas integrity concerns protecting against unauthorized modification of data. In the context of public transport, privacy often concerns not sharing any information about the travels of individuals. For instance, the movement of individual travelers can be tracked by data from mobile phone operators or through the use of RFID tags in travel cards (data which is very useful for generating Origin-Destination matrices).

In a reality where various types of sensors continuously monitor and record the activities of people and items, and where end users submit information about their activities, there are challenges of how to protect the privacy and integrity of the individuals that are being monitored. To gain the trust of the travelers it is important that no personal information is used or distributed without explicit consent.

Challenges include:

- Securing stored and communicated data against unauthorized access (both regarding disclosure and altering of the collected data), for example, using cryptography and access control in different ways.

- Anonymizing the collected data without losing the possibility to trace the activities of individuals, which might be needed to make strong analyses, for example, in order to achieve improvements of the public transport system. See Fung et al. [40] for a general survey on methods for privacy-preserving data publishing, which relates to this type of challenges. In addition, Ziegeldorf et al. [41] provide a general discussion on threats and challenges related to privacy in IoT systems.

\subsection{Security}

Unfortunately, there are many types of potential malicious actors that, for various reasons, may attack the information systems of public transport, such as cyber-terrorists and hackers. In addition, 
there are competitors, e.g., providers of public transport services that have business interests in sub-optimal operations.

The challenge related to security is quite general: How is it possible to make it more difficult for malicious actors? The state-of-the-art of security as well as privacy of IoT is covered by Sicari et al. [42].

\subsection{Interoperability}

Interoperability concerns the ability of systems to work together, which is of particular importance in machine-to-machine contexts, such as IoT, where sensors and other types of devices to a large extent communicate without the involvement of humans. As the IoT is characterized by a largely heterogeneous blend of connected devices, provided by different vendors using different types of technology, we predict that interoperability issues will constitute some of the main challenges for a well-functioning, connected IoT infrastructure.

Challenges related to interoperability include the development and usage of standardized protocols and interfaces for communication and service provision (e.g., standard web service protocols), as well as the development of efficient middleware.

\subsection{Scalability}

In the context of IoT, scalability is the ability of a system to function with growing number of users, and sensors and devices that continuously collect and process large amounts data. As mentioned earlier, the efficient management and use of this data is often referred to as "big data". Storing and processing data is not always a huge issue when the amount of data is small, but as the amount of data that needs to be stored and analyzed increases very fast with the roll-out of IoT, there are some major challenges related to the scalability, such as:

- How to store all the collected data in a way that privacy and integrity is preserved (cf. Section 3.2).

- How to analyze and process all of the collected data to transfer it into meaningful information that can be used by various types of actors, for example, travelers and transport operators.

In some cases, the amount of data is so huge that it might not even be possible to store all the collected data. In addition, privacy and integrity issues might prohibit the storage of sensitive data. Instead, there is in some situations a need to analyze data in real time.

Another challenge related to the huge amount of collected data, which perhaps cannot be stored, is how to properly aggregate data in a way that, for example, researchers can use it for future analysis. For example, statistical distributions can be an important way to store data.

There is also a need for efficient algorithms and models that can be used for analysis of the collected data and for solving problems (e.g., using simulation and mathematical optimization). Due to the complexity of many optimization problems, there is a need for efficient heuristics that can be used to solve optimization problems (often in real time).

\subsection{Usability}

In the context of IoT as an enabler for sustainable public transport, we consider usability as how easy it is for the involved actors to make use of the provided information and services (including the generation of data, e.g., via crowdsourcing). For example, the travelers typically access public transport planners through smartphone applications and interactive web pages, which need to be easy to use and to learn.

High usability is a prerequisite for achieving the benefits of IoT and is important in particular in order to reach the travelers. If the interaction is not intuitive, the services will not be used. 
Challenges related to usability include:

- How to present data and information in a way that humans and machines can easily use the information.

- How to design appropriate interaction models so that the users can interact with services and devices in an intuitive way.

For example, it is important to be able to use a public transport planner smartphone application without knowing the names of the departure and arrival bus stops.

Research and innovation within fields such as interaction design aim at facing the challenges related to usability, and general findings in this field obviously give input to building user-friendly systems within public transport.

\subsection{Data Collection}

As mentioned above, IoT can be seen as a powerful enabler of sustainable development in the context of public transport through enabling improved possibilities for data collection. However, a number of challenges are related to the actual collection of data, e.g.,

- Determining what type of data is possible to collect, both in real-time and in retrospect.

- Identifying what type of data is actually useful for the different actors in different situations.

- Collecting and storing data in the best and most efficient way. This may include non-traditional methods like crowdsourcing and using social networks like Twitter.

- How to ensure that the collected data is of sufficient quality. Data can be incorrect due to several reasons, for example, that the IoT sensors might be of poor quality or that there are individuals who intentionally want to spoof the sensors or in other ways communicate incorrect data. Hence, there is a need for methods that can be used to identify errors and inconsistencies in the collected data.

- How to handle situations with insufficient quantity of data. It is important not to base decisions on data that is too scarce.

\subsection{Deplyoment}

In order to realize the full potential of some of the opportunities listed above, it is necessary that a majority of the users have adopted the implemented IoT solutions. Thus, this challenge concerns the user acceptance of new technologies and services, which in addition to the acquisition of necessary equipment, e.g., a smartphone, may also require changed behaviors. Moreover, related to the privacy and integrity issues mentioned above, the IoT solutions usually collect data from the users through sensors or manually, requiring the users to be willing to share data.

To improve user acceptance, there have been efforts to make users involved in the design and deployment processes. For instance, the concept of living labs has recently been established as a new way of testing and evaluating new systems in a user-centered environment [43]. It can be argued that new generations of systems can be deployed in a smaller scale in a living lab in order to assess the user acceptance and technological challenges.

Obviously, not only the citizens need to change their use of technology and behavior. Also the transport providers and administrative sector need to adapt to the new technology and services.

\section{Conclusions}

We have analyzed the opportunities as well as the challenges of the fourth wave of digitalization with respect to public transport and how it can support sustainable development of society, both from environmental, economical, and social perspectives. 
The conclusion is that there are great opportunities for both transport operators and strategic transport planners, as well as, for the travelers. Some of these opportunities have been well explored, and others have a great potential to be realized.

Moreover, we have identified a number of challenges that needs to be addressed, both technical, such as data collection issues, interoperability, scalability and information security, and non-technical, such as business models, usability, privacy issues, and deployment.

From organizational perspectives there needs to be a deeper understanding of the specific needs and potential of information and ICT solutions for the different public transport stakeholders, i.e., operators, planners, different groups of customers, and public authorities.

Acknowledgments: This research was partially funded by K2-the Swedish Knowledge Centre for Public Transport, and the Knowledge Foundation through the Internet of Things and People research profile.

Author Contributions: All authors contributed equally to the research described in the article and to the writing of the article.

Conflicts of Interest: The authors declare no conflicts of interest.

\section{References}

1. Atzori, L.; Iera, A.; Morabito, G. The Internet of Things: A survey. Comput. Netw. 2010, 54, 2787-2805. [CrossRef]

2. Weiser, M. The computer for the 21st century. Sci. Am. 1991, 265, 94-104. [CrossRef]

3. Chen, M.; Mao, S.; Liu, Y. Big Data: A Survey. Mobile Netw. Appl. 2014, 19, 171-209. [CrossRef]

4. Blythe, P.; Rackliff, T.; Holland, R.; Mageean, J. ITS Applications in Public Transport: Improving the service to the transport system. J. Adv. Transp. 2000, 34, 325-345. [CrossRef]

5. Camacho, T.; Foth, M.; Rakotonirainy, A. Pervasive Technology and Public Transport: Opportunities beyond Telematics. IEEE Perv. Comput. 2013, 12, 18-25. [CrossRef]

6. Darmanin, T.; Lim, C.; Gan, H. Public Railway Disruption Recovery Planning: A new recovery strategy for metro train Melbourne. In Proceedings of the 11th Asia Pacific Industrial Engineering and Management Systems Conference, Melaka, Malaysia, 7-10 December 2010.

7. Jespersen-Groth, J.; Potthoff, D.; Clausen, J.; Huisman, D.; Kroon, L.; Maróti, G.; Nielsen, M.N. Disruption Management in Passenger Railway Transportation. In Robust and Online Large-Scale Optimization, LNCS; Ahuja, R., Möhring, R., Zaroliagis, C., Eds.; Springer International Publishing: Berlin, Germany, 2009; Volume 5868, pp. 399-421.

8. Bagchi, M.; White, P.R. The potential of public transport smart card data. Transp. Policy 2005, 12, 464-474. [CrossRef]

9. Kostakos, V.; Camacho, T.; Mantero, C. Towards proximity-based passenger sensing on public transport buses. Pers. Ubiquit. Comput. 2013, 17, 1807-1816. [CrossRef]

10. Jin, J.G.; Teo, K.M.; Odoni, A.R. Optimizing bus bridging services in response to disruptions of urban transit rail networks. Transp. Sci. 2015, 50, 790-804. [CrossRef]

11. Ibarra-Rojas, O.J.; Delgado, F.; Giesen, R.; Muñoz, J.C. Planning, operation, and control of bus transport systems: A literature review. Transp. Res. B Meth. 2015, 77, 38-75. [CrossRef]

12. Ginkel, A.; Schöbel, A. To wait or not to wait? The bicriteria delay management problem in public transportation. Transp. Sci. 2007, 41, 527-538. [CrossRef]

13. Ronald, N.; Thompson, R.; Haasz, J.; Winter, S. Determining the Viability of a Demand-Responsive Transport System under Varying Demand Scenarios. In Proceedings of the 6th ACM SIGSPATIAL International Workshop on Computational Transportation Science, Orlando, FL, USA, 5-8 November 2013.

14. Broome, K.; Worrall, L.; Fleming, J.; Boldy, D. Evaluation of flexible route bus transport for older people. Transp. Policy 2012, 21, 85-91. [CrossRef]

15. Dikas, G.; Minis, I. Scheduled paratransit transport systems. Transp. Res. B Meth. 2014, 67, 18-34. [CrossRef]

16. Nelson, J.D.; Wright, S.; Masson, B.; Ambrosino, G.; Naniopoulos, A. Recent developments in Flexible Transport Services. Res. Trans. E 2010, 29, 243-248. [CrossRef] 
17. Dias, A.; Telhada, J.; Carvalho, M.S. Simulation approach for an integrated decision support system for demand responsive transport planning and operation. In Proceedings of the 10th Annual Industrial Simulation Conference, Brno, Czech Republic, 4-6 June 2012.

18. Capriglione, D.; Liguori, C.; Pietrosanto, A. Analytical redundancy for sensor fault isolation and accommodation in public transportation vehicles. IEEE Trans. Instrum. Meas. 2004, 53, 993-999. [CrossRef]

19. Lau, S.L.; Ismail, S.S. Towards a real-time public transport data framework using crowd-sourced passenger contributed data. In Proceedings of the 82nd IEEE Vehicular Technology Conference, Boston, MA, USA, 6-9 September 2015.

20. Narla, S.R.K. The evolution of connected vehicle technology: From smart drivers to smart cars to... self-driving cars. ITE J. 2013, 83, 22-26.

21. Brownell, C.; Kornhauser, A. A Driverless Alternative-Fleet Size and Cost Requirements for a Statewide Autonomous Taxi Network in New Jersey. Transp. Res. Rec. 2014, 2416, 73-81. [CrossRef]

22. Zeng, A.Z.; Durach, C.F.; Fang, Y. Collaboration decisions on disruption recovery service in urban public tram systems. Transp. Res. E 2012, 48, 578-590. [CrossRef]

23. Stenneth, L.; Wolfson, O.; Yu, P.S.; Xu, B. Transportation mode detection using mobile phones and GIS information. In Proceedings of the 19th ACM SIGSPATIAL International Conference on Advances in Geographic Information Systems, Chicago, IL, USA, 1-4 November 2011.

24. Xia, H.; Qiao, Y.; Jian, J.; Chang, Y. Using smart phone sensors to detect transportation modes. Sensors 2014, 14, 20843-20865. [CrossRef] [PubMed]

25. Artikis, A.; Weidlich, M.; Schnitzler, F.; Boutsis, I.; Liebig, T.; Piatkowski, N.; Gal, A.; Mannor, S.; Kinane, D.; Gunopulos, D. Heterogeneous Stream Processing and Crowdsourcing for Urban Traffic Management. In Proceedings of the 17th International Conference on Extending Database Technology, Athens, Greece, 24-28 March 2014.

26. Heipke, C. Crowdsourcing geospatial data. J. Photogramm. Remote Sens. 2010, 65, 550-557. [CrossRef]

27. Andersen, A.B.; Krøgholt, P.; Bierre, S.; Tabard, A. NoxDroid on a Ride in the Inner City of Copenhagen. 2012. Available online: http://noxdroid.org/ (accessed on 27 June 2016).

28. Aoki, P.M.; Honicky, R.J.; Mainwaring, A.; Myers, C.; Paulos, E.; Subramanian, S.; Woodruff, A. Common sense: Mobile environmental sensing platforms to support community action and citizen science. In Proceedings of the 10th International Conference on Ubiquitous Computing, Seoul, Korea, 21-24 September 2008.

29. Hajinasab, B.; Davidsson, P.; Holmgren, J.; Persson, J.A. On the use of on-line services in transport simulation. In Proceedings of the International Symposium of Transport Simulation, Jeju, Korea, 29 August2 September 2016.

30. Van der Hurk, E.; Kroon, L.; Maroti, G.; Vervest, P. Deduction of Passengers' Route Choices from Smart Card Data. IEEE Trans. Intell. Transp. 2015, 16, 430-440. [CrossRef]

31. Foell, S.; Kortuem, G.; Rawassizadeh, R.; Handte, M.; Iqbal, U.; Marron, P. Micro-navigation for Urban Bus Passengers: Using the Internet of Things to improve the public transport experience. In Proceedings of the 1st International Conference on IoT in Urban Space, Rome, Italy, 27-28 October 2014.

32. Doukas, C.; Metsis, V.; Becker, E.; Le, Z.; Makedon, F.; Maglogiannis, I. Digital cities of the future: Extending @home assistive technologies for the elderly and the disabled. Telemat. Inform. 2011, 28, 176-190. [CrossRef]

33. Farkas, K.; Nagy, A.Z.; Tomás, T.; Szabó, R. Participatory sensing based real-time public transport information service. In Proceedings of the 2014 IEEE International Conference on Pervasive Computing and Communications Demonstrations, Budapest, Hungary, 24-28 March 2014.

34. Flores, G.; Cizdziel, B.; Manduchi, R.; Obraczka, K.; Do, J.; Esser, T.; Kurniawan, S. Transit Information Access for Persons with Visual or Cognitive Impairments. Computers Helping People with Special Needs. In Computers Helping People with Special Needs, LNCS; Miesenberger, K., Fels, D., Archambault, D., Peňáz, P., Zagler, W., Eds.; Springer International Publishing: Berlin, Germany, 2014; Volume 8547, pp. 403-410.

35. Garcia, C.R.; Candela, S.; Ginory, J.; Quesada-Arencibia, A.; Alayon, F. On Route Travel Assistant for Public Transport Based on Android Technology. In Proceedings of the 6th International Conference on Innovative Mobile and Internet Services in Ubiquitous Computing, Palermo, Italy, 4-6 July 2012.

36. Zuzana, B.; Bureš, P.; Jesty, P. Intelligent transport system architecture different approaches and future trends. In Data and Mobility; Düh, J., Hufnagl, H., Juritsch, E., Pfliegl, R., Schimany, H., Schönegger, H., Eds.; Springer: Berlin/Heidelberg, Germany, 2010; Volume 81, pp. 115-125. 
37. Williams, B. Intelligent Transport Systems Standards; Artech House: London, UK, 2008.

38. Dimitrakopoulos, G.; Demestichas, P. Intelligent transportation systems based on cognitive networking principles and management functionality. IEEE Veh. Technol. Mag. 2010, 1, 77-84. [CrossRef]

39. Bulger, M.; Taylor, G.; Schroeder, R. Data-Driven Business Models: Challenges and Opportunities of Big Data, Oxford Internet Institute. 2014. Available online: http://www.nemode.ac.uk (accessed on 28 August 2016).

40. Fung, B.C.M.; Wang, K.; Chen, R.; Yu, P.S. Privacy-Preserving Data Publishing: A Survey of Recent Developments. ACM Comput. Surv. 2010, 42, 7. [CrossRef]

41. Ziegeldorf, J.H.; Morchon, O.G.; Wehrle, K. Privacy in the Internet of Things: Threats and challenges. Secur. Commun. Netw. 2014, 7, 2728-2742. [CrossRef]

42. Sicari, S.; Rizzardi, A.; Grieco, L.A.; Coen-Porisini, A. Security, Privacy and Trust in Internet of Things: The road ahead. Comput. Netw. 2015, 76, 146-164. [CrossRef]

43. Schaffers, H.; Sällström, A.; Pallot, M.; Hernández-Muñoz, J.M.; Santoro, R.; Trousse, B. Integrating Living Labs with Future Internet experimental platforms for co-creating services within Smart Cities. In Proceedings of the 17th International Conference on Concurrent Enterprising (ICE), Aachen, Germany, 20-22 June 2011; pp. 1-11.

(C) 2016 by the authors; licensee MDPI, Basel, Switzerland. This article is an open access article distributed under the terms and conditions of the Creative Commons Attribution (CC-BY) license (http://creativecommons.org/licenses/by/4.0/). 



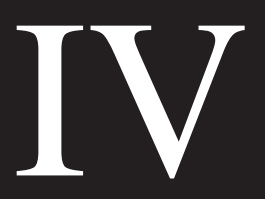



Paper 4: On the use of on-line services in transport simulation

\title{
Authors:
}

Banafsheh Hajinasab, Paul Davidsson, Johan Holmgren, Jan A. Persson

\section{Published:}

Transportation Research Procedia 21 (2017): 208-215.

\begin{abstract}
In this paper, we introduce a new approach for collecting data for transport simulation models that is using on-line services in order to outsource parts of the modeling and computation of simulation models. We describe our approach of using on-line services as part of a simulation model and we present our experiences of applying the approach to a case study using the study shows that the use of on-line services for data collection in transport simulation can bring advantages to the simulation study shows that the use of on-line services for data collection in transport simulation can bring advantages to the simulation model and improved access to the most recent information. We also noticed some limitations, such as the inability to access to information regarding the future such as timetables and no control over data provided by third-party services. However, we information regarding the future such as timetables and no control over data provided by third-party services. However, we argue that there are solutions for each of the identified limitations, and therefore we believe that the suggested approach might provide a unique opportunity for future transportation simulation models.
\end{abstract}




\title{
On the use of on-line services in transport simulation
}

\author{
Banafsheh Hajinasab $^{\text {a }}$, Paul Davidsson ${ }^{\text {a }}$, Johan Holmgren ${ }^{\text {a }}$, Jan A. Persson ${ }^{\text {a }}$ \\ ${ }^{a}$ Department of Computer Science and K2 - The Swedish Knowledge Centre for Public Transport, Malmö University, Malmö 205 06, Sweden
}

\begin{abstract}
In this paper, we introduce a new approach for collecting data for transport simulation models that is using on-line services in order to outsource parts of the modeling and computation of simulation models. We describe our approach of using on-line services as part of a simulation model and we present our experiences of applying the approach to a case study using the ASIMUT model, where the travelers between two neighbour cities in Southern Sweden are modeled. The results from our case study shows that the use of on-line services for data collection in transport simulation can bring advantages to the simulation model, for example, in terms of reduced needs for modeling of the transport system as well as computation inside the simulation model and improved access to the most recent information. We also noticed some limitations, such as the inability to access to information regarding the future such as timetables and no control over data provided by third-party services. However, we argue that there are solutions for each of the identified limitations, and therefore we believe that the suggested approach might provide a unique opportunity for future transportation simulation models.
\end{abstract}

Copyright (C) 2017 The Authors. Published by Elsevier B. V.

Selection and Peer-review under responsibility of Dept. of Transportation Engineering, University of Seoul.

Keywords: Web Services; Data Collection; Simulation; Travel planning; APIs; Transport Network

\section{Introduction}

Transportation simulation is one of the most common approaches for supporting the decision making in transportation planning through, e.g., enabling to predict the impacts of new transportation policies and infrastructure investments. Based on the questions that a simulation model needs to answer, different types of data needs to be provided to the simulation model. These data ranges from socio-demographic attributes of people to the transportation network data. The traditional approaches for preparing input for simulation models are usually about analyzing historical data, national surveys and censuses, and calculating origin-destination matrices. In traditional approaches, the data is either built into the model, or the data needs to be fed into the model manually by the modeler. The traditional data preparation approaches are complex and time-consuming since some of the data preparation work needs to be

\footnotetext{
* Corresponding author. Tel.: +46-40-66 57636

E-mail address: banafsheh.hajinasab@mah.se
} 
done manually. Furthermore, the data cannot be changed frequently due to the complex and long procedure of data preparation; therefore, these data are prepared and used as static data.

In this paper, we introduce a novel alternative approach to the traditional approaches for data collection and preparation. Since, in the suggested approach, most of the steps in the modeling and computation are done by third-party services, the input to the simulation can be changed dynamically. Moreover, on-line travel planner systems are becoming more popular these days. This means that people's travel behavior is most probably changing based on suggestions provided by these systems. Therefore, the use of on-line travel planners for estimating people's travel behavior can increase the accuracy of the travel behavior modeling in simulation systems. In this paper, we empirically investigate the advantages and limitations of our proposed approach for modeling, computation, and data preparation in an agent-based simulator for urban passenger transport (ASIMUT) ${ }^{8}$.

\section{Related work}

The predominant type of passenger transport analysis models, which are used by public authorities on regional, national, and international levels in order to support their decision-making, is the so-called macro-level models. Macrolevel models are based on highly aggregate data, and they are often described as top-down models as they are built with the purpose to reproduce known (aggregate) transport statistics, typically on national level. Examples of models of this type are the Swedish national passenger transport modeling system (Sampers) ${ }^{1}$, and TRANS-TOOLS ${ }^{9}$, which is an EU-level model for passenger and freight transport modeling ${ }^{5,12}$. Macro-level models are in general steady-state models, where time is not explicitly modeled, even though they might include components that are based on dynamic modeling. This means that, for example, bus and train departures in macro-level models are typically modeled using frequencies and average travel times instead of using timetables.

As macro-level models use aggregate data, it can be argued that the amount of data that needs to be collected, and included in the model, can be considered to be rather reasonable; at least when comparing with micro-level modes, which we discuss below. Moreover, in macro-level models, the data is in general included as a part of the model.

Micro-level models are those models where individual entities are studied over time, and they are therefore often referred to as dynamic models. Dynamic models are often used by public authorities, in order to study traffic in congested areas. Agent-based models are a special type of micro-level models, where some or more of the modeled entities are represented as agents. Agent-based models are often referred to as bottom-up models since the behavior of the modeled individuals, and the interactions between individuals are modeled and validated, and the outcome of the model is a consequence of how the individuals are modeled. An example of an agent-based passenger transport model is ASIMUT ${ }^{8}$, which we use in this paper to show the proposed data collection approach. ASIMUT is discussed further in section 4 .

From a data perspective, agent-based models (or micro-level models in general) are more data intensive, as they require data describing all the modeled entities (e.g. ${ }^{13,10,2,6,7}$ ). Obviously, it is possible to use statistical distributions in order to model the diverse behavior of the individuals, but such an approach still requires quite some effort on collecting micro-level data that represents the modeled population. As agent-based models are dynamic in nature, they also need data that allows to model entities over time, such as timetables for buses and trains. Obviously, this means that the amount of data that needs to be collected and included in the models can be quite large, in particular when studying large scenarios. Therefore, it is not always possible to build agent-based models where all of the required data is included as a part of the model, which is the case for the macro-level models discussed above.

Agent-based models are therefore sometimes modeling frameworks, which includes building blocks that can be used in order to build different analysis models. An example is the MATSim modeling framework ${ }^{3}$, which can be used in order to construct different types of passenger and freight transport models.

Through the use of modeling frameworks, it is possible to develop models using a minimum amount of data, as it is possible to tailor the data collection to the specific needs of a model. In general, data can be collected for only the modeled entities and the part of the network that are modeled. However, as the number of entities and the size of the studied geographic region grow, it becomes more and more demanding to collect the data required by a model, regardless whether or not a framework is used.

Due to the large difficulties of collecting data for agent-based (passenger transport) models, we suggest an alternative to the traditional approaches to data collection. Instead of including all required data in the model, we propose to 


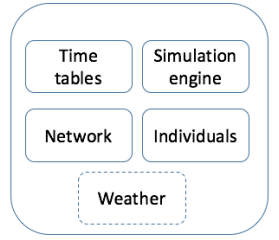

Traditional approach

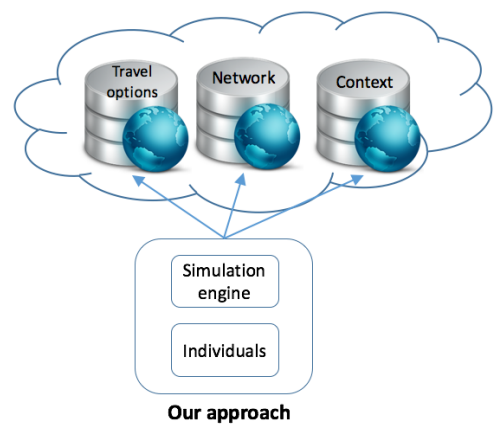

Fig. 1. The proposed approach for data collection and preparation vs traditional approaches.

outsource model computations that require data that is difficult to collect to the third-party service providers who own the data. In that way, we argue that it is possible to save considerable amount of effort on data collection, which is of particular importance when developing large models.

\section{The proposed approach}

Our proposed approach is based on outsourcing some parts of the modeling and computation of transport simulation to external on-line services. We have identified three types of data that is typically used in transport simulation and is possible to be acquired from web services. In particular, we suggest using external services to:

- Include the most promising travel options: It is possible to use web services that provide timetables and network data. In this way, the simulation model does not need to include the network and timetables data and the algorithms needed to generate travel options from these data.

- Extract data related to each travel option: To extract the data such as, time, distance, price, and emissions embedded in each of the travel options generated by the on-line services.

- Extract context data: Information about the context of the travel can also be extracted from external services, such as data about weather and nearest parking place.

In Fig. 1, we demonstrate how our proposed approach compares to the traditional approaches of data collection. As illustrated in the figure, the simulation model consists of individuals, their socio-demographic data, and the simulation engine itself. Acquiring the rest of the data required for the simulation is outsourced to the external services. In fact, part of the model related to how to calculate and obtain timetables, network, and context information, i.e. weather is outsourced to external services. In traditional models, all these data are embedded in the model, which decreases the dynamic nature of the model and the model becomes specific for the data that is built into the model.

The process of outsourcing some parts of the simulation model to external services can be a solution to some of the challenges of traditional simulation models, where all the information about the network and the timetables needs to be implemented within the model. This process is time consuming and error-prone. In addition, it requires more computational resources from the modelers' side in order to run the model. Another important challenge in traditional models is that the transport network and timetables data become obsolete after each change in transport network or after each timetable update, for example, seasonal timetable updates or addition of a new bus line. In order to address these issues, we propose a new approach where we outsource elements of a simulation model to external services. In the next sections, we illustrate our approach by describing how we applied it in an agent-based simulator (i.e., ASIMUT). 


\section{ASIMUT: Agent-based simulator for urban passenger transport}

ASIMUT is a multi-agent based simulation model that can be used as a decision support system for urban transportation planners in order to investigate the effects of different types of policy instruments and transport infrastructure investments on travel the choices of travelers ${ }^{8}$, for example, how the travelers' choices of transport would change in case of introducing a new public transport fare.

In the ASIMUT model, the travelers are explicitly modeled as agents. This enables us to model the decisions of each individual and compute the consequences of their decisions on the transport system. The relevant travel options, for each specific traveler, from an origin (point) A to a destination (point) B are generated. The relevant information about all the travel options is extracted from the web services, and the travelers choose between the generated travel options according to their individual characteristics. As mentioned above, the generated information is used in the decision making model of the travelers in order to choose which travel option is the best option for each specific traveler. The modes of transport included in the model are driving, public transport, cycling, walking, and combinations of public transport, walking and cycling.

We assume that the choices between alternatives are based on four main factors: the cost of the travel option, the travel time of the alternative, the convenience of the alternative, and the social norm. In the current version of ASIMUT $^{8}$, the convenience is defined as a combination of three factors: the number of interchanges, and the walking and cycling distances for that specific travel option. Also, social norms are limited to how much a traveler cares about the environmental consequences of her travel. The amount of $\mathrm{CO}_{2}$ emissions emitted is used as an indicator for how much a specific travel damages the environment.

Furthermore, we believe that the decision-making process of the travelers, when choosing between the available travel options is to some extent individual and not the same for all travelers. Therefore, we assume that the best travel option can be different for different travelers according their characteristics and contextual factors, e.g., weather conditions. The individual characteristics in ASIMUT are age, income, work flexibility, and eco-friendliness. As an example, the perceived value (significance) of the cost factor is different for each traveler and depends on the amount of income of the traveler. We also use other information about travelers such as work and home address, working hours, access to car, and access to bicycle at home and at work, when generating and filtering the relevant travel options.

Using a utility function, we calculate a score for each of the travel options of the individuals. It should be emphasized here that the calculated score actually represents the disutility of a travel option; therefore, ASIMUT will always choose the travel option with the lowest score among the set of available options for each traveler agent (indiviudual).

\section{Case study: Using web services for data collection in ASIMUT}

Our proposed approach has been used as part of the ASIMUT model for data collection, preparation, and outsourcing of computations. In particular, we used on-line travel planners to generate travel options from the origin to the destination of each of the modeled travelers. In addition, we used a web forecasting service in order to collect the weather data used in the traveler decision making model. Instead of modeling the transport system explicitly, we used on-line web services in order to generate travel options in ASIMUT.

The set of travel options are generated by sending several requests to the APIs of the local travel planner, i.e. Skånetrafiken ${ }^{\mathrm{a}}$ and Google Maps Directions web service ${ }^{\mathrm{b}}$. After getting all the travel options of the travelers, we extract some information about each travel option, which is important in the decision-making model of the travelers. To summarize, the following information related to each travel option are extracted from the on-line services:

- Cost; refers to the cost for the travel option. In case of public transport, the cost is the ticket price for the route, while for driving, the cost refers to the fuel cost plus the parking costs. The cost for cycling and walking is assumed to be zero.

\footnotetext{
a https://www.skanetrafiken.se/

${ }^{\mathrm{b}}$ https://developers.google.com/maps/documentation/directions/
} 
- Travel time; refers to the door to door travel time from the origin to the destination.

- $\mathrm{CO}_{2}$ emission; the amount of $\mathrm{CO}_{2}$ emissions emitted from each travel option.

- Number of interchanges; the number of times that the traveler needs to change the mode of transport.

- Walking distance; the total walking distance of the alternative.

- Cycling distance; the total cycling distance of the alternative.

As mentioned above, we used a combination of a local on-line travel planner, i.e. Skånetrafiken, and Google Maps in order to build travel options and extract the parameters (e.g. cost and $\mathrm{CO}_{2}$ ) for each of the options. It is obvious that these on-line services can be replaced by any other on-line service that is relevant for the geographic region of a particular case study. Furthermore, the proposed approach for outsourcing data collection and modeling to web services can be used in any simulation model that needs to collect data about network, public transport schedules, etc. In general, the approach can be used to collect any other data that is needed.

At the first stage, ASIMUT generates two travel requests for each traveler according to their origin and destination coordinates and to their working schedule, i.e., one travel request for going from home to work and another request for going from work back to home. Thereafter, for each travel request, we generate the most promising travel options using different modes of transport. The sequence of collecting data and generating the travel options from web services in the ASIMUT model is shown in Fig. 2. Walking, cycling, and driving travel options refer to the options that use only one of walking, cycling, and driving as the only mode of transport all the way from the origin A to the destination B. ASIMUT sends a request to the Google Directions API in order to get bike travel options for the whole distance between A and B. The Google Directions API returns the bike routes and ASIMUT stores the bike routes data. In the same way (as for biking routes), the Google Directions API generates walking and driving routes.

In Fig. 2, the public transport options refer to the travel options that use public transport together with some short walking to and from public transport stops. The travel time and distance of these short walks are taken into account in the simulation. The public transportation options are requested from the Skånetrafiken API and the data is stored by ASIMUT. In some on-line travel planners, such as Skånetrafiken API, which we used in our case study, it is not possible to combine biking and public transport. Since it is very likely that travelers bike or walk to the nearest bus/train station, we generate those travel options that are not suggested by the Skånetrafiken API inside ASIMUT. In this way, we try to cover all possible travel options including the ones that combine bike and public transport, i.e., those that are not generated by on-line services used in the study. For this purpose, we replace long walking distances from origin (A) to a station (A), and from a station (B)́ to destination (B) by cycling. Long walking is defined as the walking distances (d) between 200 meters and 6000 meters. In order to generate these extra options, we send new requests to the Google Directions API to get the biking options, i.e. biking options from (A) to (A), and from (B) to (B). It should be noted that these options will be generated only if the traveler has access to bike at the origin and/or at the destination. Finally the weather data is requested and collected to be used later for route selection in the traveler decision model.

We developed a simple scenario in ASIMUT to investigate the effect of changes to the public transport cost on the travelers' choice of mode of transport, the total amount of $\mathrm{CO}_{2}$ emission, the total travel time, and the total cost. The scenarios and details of the model are described in a previous paper ${ }^{8}$. The process of the simulation runs and the results of the study proved the possibility of using web services for data collection in a simulation model.

In the following sections, we describe how we used each of the external web services to prepare the input data for ASIMUT, i.e. Skånetrafiken travel planner, Google Maps Directions API, and a weather forecasting service called Weather Underground ${ }^{\mathrm{c}}$

\subsection{Skånetrafiken travel planner}

Skånetrafiken is an administration organization responsible for public transport in the region of Skåne in southern Sweden. The organization provides a travel planner called Skånetrafiken Reseplaneraren (In English: Scania county's Travel planner) in which travelers can get the available public transport options for their travel needs. The service is available through a website and as a mobile phone application for direct access by the travelers. The travel planner

\footnotetext{
${ }^{\mathrm{c}}$ https://www.wunderground.com/
} 


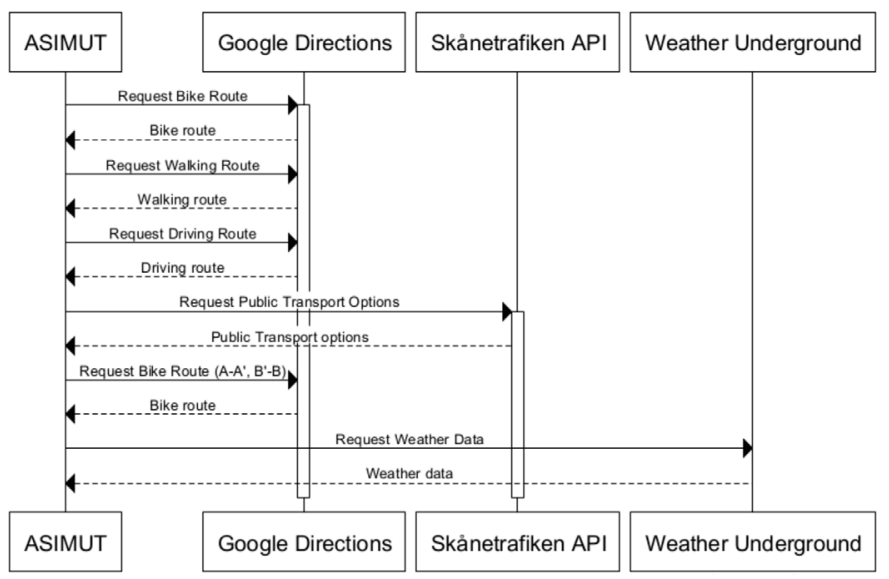

Fig. 2. Sequence of the ASIMUT model.

is also available via an open API for developers of transport-related information systems. The communication in this case is machine to machine via exchanging XML files.

The API used in this service is SOAP based and returns an XML file as response. SOAP (Simple Object Access Protocol) is as an XML-based protocol for exchanging information when accessing web services, in this case exchanging data related to public transport options. In particular, a SOAP message is an XML file that consists of the encoding rules and the message itself ${ }^{4}$. The Skånetrafiken API provides a number of methods for accessing different information. Some of the functionalities provided by this API methods are to search for: available travel options, stops/stations, stops around a given point, departure times from a stop/station, and current modes of transport. We provide these information to the service: geographical coordinates of the origin and the destination, and departure or arrival time of each of the travelers. The information that we extract from the output of the web service and that we use as input to the ASIMUT model are: travel time, cost, $\mathrm{CO}_{2}$ emission, number of interchanges, and walking distance for each of the suggested travel options.

\subsection{Google Maps Directions API}

Google maps provides a RESTful API that returns travel options for different transport modes between two points. We have used the API to generate walking, cycling and driving travel options. The geographical coordinates of each travelers' origin and destination, and the preferred departure time are sent as input to the service. For each of the travel options, the output is the travel time and the distance for each of the travel options.

\subsection{Weather data}

In order to access to the predicted weather data, we used a web service provided by the Weather Underground. The information about the temperature and weather condition (rainy, snowy, sunny) which is collected from this service, is used in the ASIMUT model as a determining factor in the travel choice prediction model.

\section{Discussion \& Conclusions}

Our empirical study showed that the use of on-line travel planners for generating travel options enables us to capture the most recent information about route alternatives and their relevant characteristics such as cost and travel 
time. Moreover, it provides the model with real-time information that adapts automatically with updates. For instance, if the bus schedules change, this change will be automatically updated in the simulation model.

Due to the increasing popularity of the on-line travel planners, most travelers use on-line travel planners in order to retrieve almost all possible travel options at the time of departure. Therefore, we believe integrating web services provided by on-line travel planners with the transportation simulation models makes the model very close to the reality and reflects a more realistic picture of the travelers' behavior.

Furthermore, on-line services provide a unique opportunity to outsource the data collection, modeling, and computational work to third-parties ${ }^{11}$. In this case, the calculation of routes and all the relevant information about the routes is done by the travel planners' web services.

Apart from the above-mentioned advantages, our proposed approach has also some limitations. First of all, since the existing web services are designed to provide service to the users in real-time, they have limitations in providing historical and future data. In most cases, on-line travel planners do not provide travel information for travels that occur in the far future, e.g. more than 3 months ahead, even in case the timetables exist for future services. However, since the idea of the approach is to predict the consequences of a change in the transportation system, depending on the studied scenario, we argue that it is possible to use the current travel options and just change the factor that is being studied.

Second, in our approach the simulation model does not have any control over the output data provided by web services because the data is generated dynamically by the web services in real-time. This means, it is difficult to conduct controlled simulations. For example, in case we need to compare two different scenarios, the results might be affected just because we run the simulation at two different times. The same problem occurs when there is a need to repeat an experiment at a later point of time. The results we get might change from date to date. This limitation can be addressed by storing the travel data in a database locally to be used in future simulation runs. In this way, it is possible to do a controlled experiment using the stored data.

Third, the scalability of our approach might be limited by the capacity of the services provided by third parties, such as the Google API or the local travel planners. It is often the case that the servers that provide the web services have limitations in accepting requests and returning output without delays. The relevant public transport authorities typically use simulation models in order to support their decision-making, therefore they most likely would benefit from such a simulation model based on our approach and from the results it generates. Hence, it is most likely the case that they are willing to provide a special server for such a simulation model.

Finally, all the possible travel options that travel planners want to investigate in a simulation scenario are not necessarily covered by on-line travel planners (e.g. Skånetrafiken). To overcome this limitation, it is possible to let the model generate additional travel options based on the requirements of the simulation scenario, which is exactly what we did in ASIMUT (i.e. by adding travel options that combine public transport and cycling).

In general, we believe this new approach for data collection, preparation, and modeling can be an alternative approach to the existing modeling approaches. This new method can facilitate the process of modeling by outsourcing parts of the model to the third-parties and can increase the quality of the simulation by obtaining the most recent information.

\section{Acknowledgements}

We thank K2- The Sweden's national center for research and education on public transport for partially funding this research, and the National ITS Postgraduate School for their support.

\section{References}

1. S. Algers, L.-G. Mattsson, C. Rydergren, and B. Östlund. Sampers: erfarenheter och utvecklingsmöjligheter på kort och lång sikt. 2009.

2. S. An, J. Cui, and L. Li. Agent-based approach to model commuter behaviour's day-to-day dynamics under pre-trip information. Intelligent Transport Systems, IET, 5(1):70-79, 2011.

3. M. Balmer, M. Rieser, K. Meister, D. Charypar, N. Lefebvre, K. Nagel, and K. Axhausen. Matsim-t: Architecture and simulation times. Multi-agent systems for traffic and transportation engineering, pages 57-78, 2009.

4. D. Box, D. Ehnebuske, G. Kakivaya, A. Layman, N. Mendelsohn, H. Frystyk Nielsen, S. Thatte, and D. Winer. Simple Object Access Protocol (SOAP) 1.1, 2000 (accessed April 10, 2016) 
5. A. Burgess, M. Snelder, A. Martino, D. Fiorello, J. Bröcker, N. Schneekloth, and L. Rudzi-kaite. Trans-tools (tools for transport forecasting and scenario testing) deliverable 1. Funded by 6th Framework RTD Programme, TNO INRO, Delft, The Netherlands, 2006.

6. H. Dia. An agent-based approach to modelling driver route choice behaviour under the influence of real-time information. Transportation Research Part C: Emerging Technologies, 10(5):331-349, 2002.

7. D. Grether, Y. Chen, M. Rieser, U. Beuck, and K. Nagel. Emergent effects in multi-agent simulations of road pricing. In Proceedings of the Annual Meeting of the European Regional Science Association (ERSA), pages 08-08, 2008.

8. B. Hajinasab, P. Davidsson, J. A. Persson, and J. Holmgren. Towards an agent-based model of passenger transportation. In Lecture Notes in Computer Science, Multi-Agent Based Simulation XVI, volume 9568, pages 132-145. Springer, 2015.

9. C. Hansen. Report on scenario, traffic forecast and analysis of traffic on the ten-t, taking into consideration the external dimension of the uniontrans-tools model version 2: Calibration and forecasts 2020 and 2030. Recent Developments in Transport Economics, 2009.

10. S. McDonnell and M. Zellner. Exploring the effectiveness of bus rapid transit a prototype agent-based model of commuting behavior. Transport Policy, 18(6):825-835, 2011.

11. M. Papazoglou. Web services: principles and technology. Pearson Education, 2008.

12. J. Rich, J. Bröcker, C. H. Overgård, A. Korzenewych, O. A. Nielsen, and G. Vuk. Report on scenario, traffic forecast and analysis of traffic on the ten-t, taking into consideration the external dimension of the union: Trans-tools version 2; model and data improvements. Technical report, 2009

13. J. Wahle, A. L. C. Bazzan, F. Klügl, and M. Schreckenberg. The impact of real-time information in a two-route scenario using agent-based simulation. Transportation Research Part C: Emerging Technologies, 10(5):399-417, 2002. 



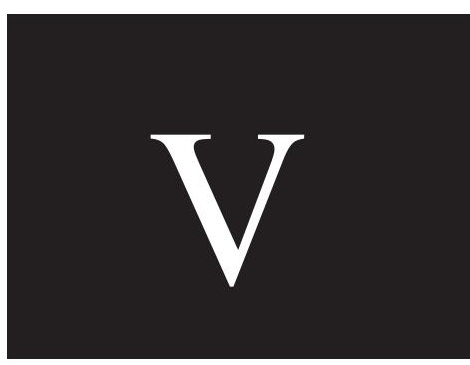





\title{
An on-line agent-based approach to estimate the effects of transport policy instruments: An empirical study of commuting in urban areas
}

\author{
Banafsheh Hajinasab ${ }^{\mathrm{a}, \mathrm{b}}$, Paul Davidsson ${ }^{\mathrm{a}, \mathrm{b}}$, Jan A. Persson ${ }^{\mathrm{a}, \mathrm{b}}$ \\ ${ }^{a}$ Department of Computer Science, Sweden, Malmö University, SE 20506 Malmö, Sweden \\ ${ }^{b} K 2$ - The Swedish Knowledge Centre for Public Transport, Scheelevägen 2, SE 22381 Lund, Sweden
}

\begin{abstract}
This paper reports an empirical study on using on-line travel planners in agent-based simulation for predicting effect of different policy instruments on travel behavior. First, we developed a general agent-based model for passenger transport including cycling and walking and then, as a case study, we investigated the important policy instruments in a certain geographical area. We used on-line services as a new way of generation and use of open data in transport modelling which decreases the computational need and increases the flexibility and dynamicity of the model. The results from our empirical study in three different urban commuting scenario between two neighbour cities in southern Sweden showed that our general-purpose agent-based model facilitates simulation of different policy instruments specially scenarios where the infrastructure does not change. Moreover, we found out that the use of on-line services for data collection increases the speed and flexibility of the system for defining and running new scenarios. However, the scalability of using on-line services in simulation may be constrained by limitations of on-line service providers. (C) 2017 .
\end{abstract}

Keywords: Urban transport planning; Web Services; Data Collection; Simulation; Calibration; APIs; Transport Network

\section{Introduction}

Simulation is one of the most prominent approaches for analyzing impacts of different scenarios in transportation. Decision makers use transportation simulation to predict impact of different policies or infrastructure investments on travellers' behaviour. However, modelling travellers' behavior in an unpredictable world is a very complex issue ${ }^{7}$. The most dominant paradigm for modelling travel behavior in transportation research is rational choice where a traveller chooses an alternative which maximizes her utility. Utility is calculated based on the attributes of a travel alternative, for instance travel cost and time, comfort, environmental friendliness, etc. A discrete choice analysis approach can be used to model travel behavior, for instance, for modelling mode and route choices ${ }^{5}$. In addition due to simplicity and mathematical elegance of the utility-based models, these models handle many unknown factors affecting individual choice by their flexible structures of error ${ }^{24}$. However, there is always a main critique for rational choice approach that is to be normative which means the model explains how rational individuals would decide, not how actual travelers decide. The real decision making is affected by many factors such as different types of uncertainty, emotions, lack of having all needed information, different perception of risks for each travel alternative, etc. ${ }^{7}$. To overcome this problem a lot of different approaches (e.g. adopting context-dependent heuristics ${ }^{11}$, Prospect Theory ${ }^{30}$, law of effect ${ }^{8}$ ) has been

\footnotetext{
* Corresponding author. Tel.: +46-40-66 57636
} 
proposed by the transportation research community for providing a more realistic model of human decision making. But most of these models assume that the human needs to gain and process all the information needed for decision making. Thanks to the recent advances in ICT technologies, today travellers have access to the real-time information anytime and anywhere through mobile devices and a lot of different sources such as on-line travel planners ${ }^{29}$. People are increasingly using on-line services to get prescriptive information for planning their travels before departure. Research has shown that travellers have an increasing tendency to rely on prescriptive information and not decide in the dark ${ }^{6}$. It can be predicted that travellers' behavior will increasingly be changing towards what on-line travel planners prescribe. This opens new opportunities to use the output of on-line travel planners in simulation systems for modelling travel behavior ${ }^{13}$. Apart from the important effect of on-line travel planners on travel behavior, using output of on-line travel planners can automatize part of the data preparation for simulation. As we know transportation simulation is a data-intensive process that requires a huge amount of input ranged from transportation network model to the socio-demographic attributes of travellers. These data are usually collected through cumbersome and timeconsuming procedures such as surveys, analyzing historical data, and calculating origin-destination matrices. As a result, it will be very difficult and time consuming to change the input data to the simulation model frequently. In this paper, we have empirically investigated the feasibility and limitations of using on-line travel planners in an agentbased simulation of travellers' behavior in an urban commuting scenario between two neighbor cities in southern Sweden. First of all, we developed an agent-based decision making model for passenger transport policy analysis ${ }^{15}$. Second, we have investigated the importance of different transport policy instruments with regard to affecting travel behavior through a survey among transport decision makers. Third, we calibrated the adopted model using sample data collected from real commuters between two cities in southern Sweden and data acquired from on-line travel planners (Google Maps and Skånetrafiken web services) and other on-line services. Finally, the calibrated model is used to study the effects of some hypothetical scenarios that were ranked as important in our initial survey. We also address some limitations of using on-line services approach.

\section{Related work}

In general, passenger transport analysis models fall into two main categories: 1) macro-level (top-down) models that use highly aggregated data to reproduce known transport statistics, typically on a national level (e.g. in the following studies ${ }^{1},{ }^{17}$ ), and 2) micro-level models where individual entities are studied over time. Micro-level models are therefore often referred to as dynamic models. In the area of transportation modelling, dynamic models are mainly used to study traffic congestion. One of the special types of micro-level models is agent-based where some entities are represented as agents. Agent-based models follow a bottom-up approach where the behavior of each individuals and the interactions between them are modelled e.g. the following study ${ }^{16}$. The macro-level models have the advantage of using a reasonable amount of aggregated data for transport analysis in comparison with micro-level models such as agent-based models that usually need a relatively large amount of data about each agent. However, macro-level models are in general steady-state models while micro-level models (e.g. agent-based models) are dynamic in nature that allows to model entities over time ${ }^{25}$. In fact, time is an important aspect in studying many transport policy measures such as time-differentiated congestion and parking fees, and it is not easy to use traditional top-down models for investigating the effect of such policies on travel behavior. On the contrary, agent-based models allow for modelling each individual as an agent who takes travel decisions dynamically over time ${ }^{28,12}$. In this way, travel decisions of each individual can be affected by adding different transport policy measures to the simulation ${ }^{16}$.

While the conventional top-down models (i.e. discrete choice models) are still the dominating approach for modelling travel behavior, they only cover a limited number of the factors influencing travel behavior ${ }^{14}$. An example of such models is the four-step modelling approach. The four steps are: 1) trip generation, determining the frequency of trips between zones; 2) trip distribution, matching origins with destinations; 3) mode choice, computing proportion of each transport mode in each trip; and 4) route assignment, assigning trips to routes. Four-step models have been criticized for oversimplification and ignoring the interaction between the involved actors that often lead to significant biases in output ${ }^{27}$.

Despite the great potential of agent-based approach in modelling the effect of transport measures on travel behavior, there are few studies that have applied an agent-based modelling approach in the context of transport policy analysis ${ }^{14}$. Most of these models ${ }^{23,2}$ only demonstrate some potentials of the agent-based approach. These models are mainly 
proposed to study a specific transport policy measure in a particular scenario, and they do not necessarily include all transportation modes. In fact the input variables, the model, and the output are defined to solve only a particular scenario. Therefore, these models cannot be used as a general model to investigate the effects of different types of transport policy measures in various scenarios. ASIMUT ${ }^{16}$ is an example of a general agent-based model for analyzing different transport measures in various scenarios. ASIMUT includes both motorized and non-motorized modes of transportation and the combinations of them for generating travel alternatives. The model focuses on the mode choice, route choice and departure time choices of travellers, when source and destination data is given, e.g., the traveller's home and work addresses. However, it does not model the vehicles that travelers are being on, since it is out of scope of the model.

All in all, it seems agent-based modelling is a more promising approach to investigate the effect of transport policy measures on travel behavior compared to top-down conventional approaches. However, even in an agent-based model, modelling travellers' behavior can be a big challenge due to the uncertainty in different parameters 7 . Different approaches have been proposed to model human decision making in an unpredictable world.

For example, adopting context-dependent heuristics ${ }^{4}$ that focuses on limitation of human cognition. In this approach, humans are assumed to be rationally bounded which means they cannot acquire and process all information about all possible alternatives ${ }^{11}$. Instead they focus on the most important attributes and apply simple heuristics to take decision ${ }^{26}$. Prospect Theory ${ }^{30}$ is another approach that allows for a subjective weighting of perceived probabilities reflecting the decision maker's attitude to risk. According to Prospect Theory, decisions are context-dependent and evaluation of risky prospects includes assessment of behavioral outcomes with regard to some common reference points. The reference points change from one context to the other and over time which means that information provided to the travellers in different steps e.g. pre-travel or en rout can have different effects on their behavior. Finally, approaches that rely on the law of effect ${ }^{8}$ take into consideration that people usually recall extraordinary and particularly bad events and forget unremarkable or normal occasions. The travellers are more likely to recall the experience of missing a train or being late for the meeting than the ones when everything worked according to the plan. This means that travellers rely on their past experience to from a knowledge on the distribution of outcome that can be far from a rational choice.

However, the most common paradigm is still rational choice approach where the (dis)utility of different travel alternatives determines traveller's choices. Utility-based approaches are simple and can handle unseen factors due to their flexible error structure ${ }^{24}$. In this paper we have further developed the travel behavior model of ASIMUT ${ }^{16}$ that is a utility-based model for an agent-based simulation where the utility of a travel choice is calculated based on the traveller's characteristics, the available travel options, and the contextual factors (e.g. weather). Since in ASIMUT the transport network data is not hard coded, it is more flexible and adaptable in investigating the effects of different policies in various scenarios. ASIMUT is developed from scratch in Java, but there are also frameworks and platforms such as GAMA ${ }^{9}$ and MATSIM ${ }^{3,19}$ which have promising possibilities for developing agent-based simulations. Through the use of modeling frameworks, it is possible to develop models with a minimum amount of data, as it is possible to tailor the data collection to the specific needs of a model. In general, data can be collected for only the modeled entities and the part of the network that are modeled. However, as the number of entities and the size of the studied geographic region grows, it becomes more and more demanding to collect the data, regardless whether or not a framework is used. Therefore, the approach of using on-line services as part of data collection can be beneficial in many cases. For this reason, we used ASIMUT in this paper in order to have freedom in development specially for implementing the approach of using web services as part of the model.

\section{ASIMUT: Agent-based simulator for urban passenger transport}

ASIMUT is a multi-agent based simulation model that can be used as a decision support system for urban transportation planners in order to investigate the effects of different types of policy instruments and transport infrastructure investments on travel choices of travellers ${ }^{16}$, for example, how the travellers' choices of transport would change in case of introducing a new public transport fare.

In the ASIMUT model, the travellers are explicitly modelled as agents. This enables us to model the decisions of each individual and compute the consequences of their decisions on the transport system. The relevant travel options, for each specific traveller, from an origin (point) A to a destination (point) B are generated. The relevant information 
about all the travel options is extracted from the web services, and the travellers choose between the generated travel options according to their individual characteristics and the weather. As mentioned above, the generated information is used in the decision making model of the travellers in order to choose which travel option is the best option for each specific traveller in a specific situation. The modes of transport included in the model are driving, public transport, cycling, walking, and combinations of public transport, walking and cycling.

We assume that the choices between alternatives are based on four main factors: the cost of the travel option, the travel time of the alternative, the convenience of the alternative, and the social norm. In the current version of ASIMUT $^{16}$, the convenience is defined as a combination of three factors: the number of interchanges, the walking distance and the cycling distance for each travel option. Also, social norms are limited to how much a traveller cares about the environmental consequences of her travel. The amount of $\mathrm{CO}_{2}$ emissions emitted is used as an indicator for how much a specific travel damages the environment.

Furthermore, we believe that the decision making process of the travellers, when choosing between the available travel options is to some extent individual and not the same for all travellers. Therefore, we assume that the best travel option can be different for different travellers according to their characteristics and contextual factors, e.g., weather conditions (weather is represented as $v_{a l}{ }_{t}^{\text {wth }}$ in equation (1). The individual characteristics in ASIMUT are age, income, work flexibility, and eco-friendliness. As an example, the perceived value (significance) of the cost factor is different for each traveller and depends on the amount of income of the traveller. We also use other information about travellers such as work and home address, working hours, access to car, and access to bicycle at home and at work, when generating and filtering the relevant travel options.

$$
\begin{aligned}
& S_{\text {oat }}=W_{\text {cost }} * \text { rel }_{\text {oat }}^{\text {cost }} * v_{\text {val }}^{\text {income }}+W_{\text {time }} * \text { rel }_{\text {oat }}^{\text {time }} * \text { val }_{a}^{\text {workFlex }}+
\end{aligned}
$$

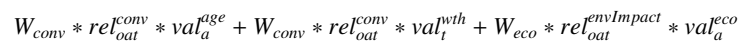

Using a utility function (illustrated in equation (1), we calculate a score for each of the travel options of the individuals. The factor 'convenience' is determined by combination of the three factors of walking distance, cycling distance, and the number of changes of the travel option $o$ for agent $a$ in ASIMUT, and it is calculated as shown in equation (2).

$$
r e l_{\text {oat }}^{c o n v}=r e l_{\text {oat }}^{\text {wlkDis }}+r e l_{\text {oat }}^{c y c D i s}+r e l_{\text {oat }}^{\text {noOfChange }}
$$

The values of relative time and cost are calculated by normalizing the cost, time, and environmental impact of a travel option with respect to the other travel options of traveler $a$ for trip $t$, as shown in the equations $(3,4,5)$ in which $O$ refers to the collection of all travel options of trip t for traveler $a$.

$$
\begin{aligned}
r e l_{\text {oat }}^{\text {cost }} & =\frac{\text { Cost }_{\text {oat }}}{\sum_{o^{\prime} \epsilon O} \text { Cost }_{o^{\prime} a t}} \\
r e l_{\text {oat }}^{\text {time }} & =\frac{\text { Time }_{\text {oat }}}{\sum_{o^{\prime} \epsilon O} \text { Time }_{o^{\prime} a t}} \\
\text { rel }_{\text {oat }}^{\text {envImpact }} & =\frac{\text { Co2Emission }_{\text {oat }}}{\sum_{o^{\prime} \epsilon O} \text { Co2Emission }_{o^{\prime} a t}}
\end{aligned}
$$

The same normalization process applies to the factors for convenience, as shown in the equations $(6,7,8)$, e.g. in order to calculate the relative environmental impact of a travel option $o$, the CO_2 emission of that travel option is divided by the sum over the CO_2 emissions of all the travel options $o$ for trip $t$ of the agent $a$.

$$
\begin{aligned}
& \text { rel }_{\text {oat }}^{\text {wlkDis }}=\frac{\text { WalkingDistance }_{\text {oat }}}{\sum_{o^{\prime} \epsilon O} \text { WalkingDistance }_{o^{\prime} a t}} \\
& \text { rel }_{\text {oat }}^{\text {cycis }}=\frac{\text { CyclingDistance }_{\text {oat }}}{\sum_{o^{\prime} \epsilon O} \text { CyclingDistance }_{o^{\prime} a t}}
\end{aligned}
$$

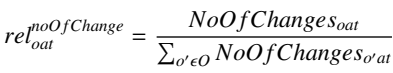


Table 1: The categorization of characteristics of travellers and contextual factor $\left(\mathrm{Val}_{a}^{x x}\right.$ and $\left.\mathrm{Val}_{t}^{\text {wth }}\right)$

\begin{tabular}{|c|c|c|}
\hline Variable & Range & Value \\
\hline \multirow{5}{*}{ Age } & $15-25$ & 0.1 \\
\hline & $25-35$ & 0.3 \\
\hline & $35-55$ & 0.5 \\
\hline & $55-70$ & 0.7 \\
\hline & +70 & 0.9 \\
\hline \multirow{5}{*}{ Income (monthly) } & +100000 & 0.1 \\
\hline & $50000-100000$ & 0.3 \\
\hline & $25000-50000$ & 0.5 \\
\hline & $15000-25000$ & 0.7 \\
\hline & $<15000$ & 0.9 \\
\hline \multirow{3}{*}{ Work Flexibility } & high & 0.4 \\
\hline & average & 0.5 \\
\hline & low & 0.6 \\
\hline \multirow{3}{*}{ Eco-friendliness } & not concerned & 0.3 \\
\hline & medium engagement & 0.5 \\
\hline & high engagement & 0.7 \\
\hline \multirow{3}{*}{ Weather } & Good (no rain or snow, and temp $>10^{\circ} \mathrm{C}$ ) & 0.2 \\
\hline & Average (no rain or snow and temp $0-10^{\circ} \mathrm{C}$ ) & 0.5 \\
\hline & bad (rain or snow, or temp $<0^{\circ} \mathrm{C}$ ) & 0.8 \\
\hline
\end{tabular}

In ASIMUT decision making model, the real values for the age, in-come, work flexibility, environmental awareness (i.e., eco-friendliness), and weather characteristics are translated into categories as shown in the Table 1 in the value column. These translations are the values used in the utility function, i.e., $V a l_{a}^{x x}$ and $V a l_{t}^{w t h}$, and they are all numbers between 0 and 1 . It should be noted that the values used in the scoring function are just preliminary estimations; they will be further analyzed and validated in future studies. It should be emphasized here that the calculated score actually represents the disutility of a travel option; therefore, ASIMUT will always choose the travel option with the lowest score among the set of available options for each traveller agent (individual).

\subsection{Using on-line travel planners to generate travel option data}

As we mentioned earlier we have used on-line travel planners and other web services to prepare rest of the input data that we need for simulation ${ }^{13}$. We generated travel options by sending several requests to the APIs of the local travel planner, i.e. Skånetrafiken ${ }^{\mathrm{a}}$ and Google Maps Directions web service ${ }^{\mathrm{b}}$. After receiving all travel options, we extracted the information which is important in our travel behavior model. To summarize, the following information are extracted from the on-line services for each travel option:

1. Cost: refers to the cost for the travel option. In case of public transport, the cost is the ticket price for the route, while for driving, the cost refers to the cost of using car per kilometer including fuel cost, parking costs, etc. The cost for cycling and walking is assumed to be zero.

2. Travel time: refers to the door to door travel time from the origin to the destination.

3. CO_2 emission: the amount of CO_2 emissions emitted from each travel option.

4. Number of interchanges: the number of times that the traveller needs to change the mode of transport. In case the travel option includes walking in between public transport modes to change to the other public transport (e.g. change from train to bus) or walking to parking place to get the car, those walking segments are not considered as change of mode.

\footnotetext{
a https://www.skanetrafiken.se/

${ }^{b}$ https://developers.google.com/maps/documentation/directions/
} 


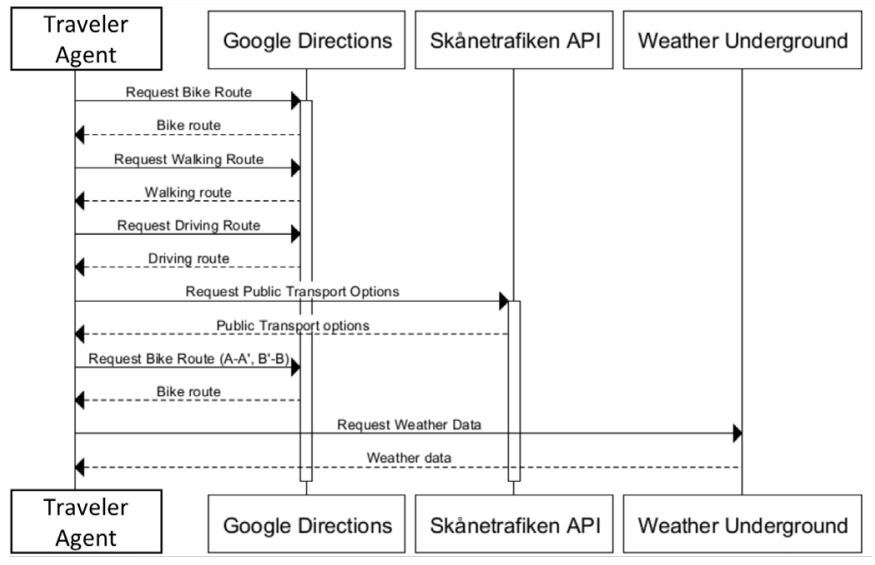

Figure 1: Sequence of the ASIMUT model.

5. Walking distance: the total walking distance of the alternative.

6. Cycling distance: the total cycling distance of the alternative.

We also used a third party web service for providing weather forecast data to the ASIMUT travel behaviour model.

At the first stage, ASIMUT generates two travel requests for each traveller according to their origin and destination coordinates and to their working schedule, i.e., one travel request for going from home to work and another request for going from work back to home. Thereafter, for each travel request, we generate the most promising travel options using different modes of transport. The sequence of collecting data and generating the travel options from web services in the ASIMUT model is shown in Fig. 1. Walking, cycling, and driving travel options refer to the options that use only one of walking, cycling, and driving as the only mode of transport all the way from the origin A to the destination B. Traveler agent sends a request to the Google Directions API in order to get bike travel options for the whole distance between A and B. The Google Directions API returns the bike routes and the traveler agent stores the bike routes data. In the same way (as for biking routes), the Google Directions API generates walking and driving routes.

In Fig. 1, the public transport options refer to the travel options that use public transport together with some short walking to and from public transport stops. The travel time and distance of these short walks are taken into account in the simulation. The public transportation options are requested from the Skånetrafiken API and the data is stored by ASIMUT. In some on-line travel planners, such as Skånetrafiken API, which we used in our case study, it is not possible to combine biking and public transport. Since it is possible that travellers bike or walk to the nearest bus/train station, we generate those travel options that are not suggested by the Skånetrafiken API inside ASIMUT. In this way, we try to cover all possible travel options including the ones that combine bike and public transport, i.e., those that are not generated by on-line services used in the study. For this purpose, we replace long walking distances from origin (A) to a station (A), and from a station (B)́ to destination (B) by cycling. Long walking is defined as the walking distances (d) between 200 meters and 6000 meters. In order to generate these extra options, we send new requests to the Google Directions API to get the biking options, i.e. biking options from (A) to (A), and from (B) to (B). It should be noted that these options will be generated only if the traveller has access to bike at the origin and/or at the destination. Finally the weather data is requested and collected to be used later for route selection in the traveller decision model. 


\section{Empirical study: simulation of travellers' behaviour in commuting between two neighbour cities in Sweden}

To investigate the feasibility of using on-line travel planners in an agent-based simulation for predicting the effect of policy measures on travel behavior and study the potential scenario cases, we conducted an empirical study in southern Sweden.

\subsection{A survey on priorities of transport policy instruments in southern Sweden}

\subsubsection{Goals of the survey}

Transport planning process starts with a high-level goal which describes the aims that the transport planners ultimately want to achieve, e.g. to improve accessibility. At the next level, the transport planers establish some specific and achievable objectives. Some example objectives that contribute to the mentioned sample goal can be: to reduce traffic congestion delay or to reduce barriers to non-motorized travel. The last stage includes the selection of policy instruments in order to achieve the objectives ${ }^{21}$.

Simulation can be a valuable approach to predict the effects of different policy instrument in the context of transport planning. To design a useful and applicable simulation tool for transport planners and policy makers, we need to understand their needs. We need to understand what kind of policy instruments and scenarios they want to investigate and which kind of potential effects they want to study. This helps us to design better simulation models that fits to the requirements of transport planers.

\subsubsection{Method of the survey}

We have conducted a questionnaire study where we have asked 21 policy makers and researchers of public transportation in Sweden about the possible requirements of a transport model. The respondents are selected based on their expertise and area of work which was relevant to urban transport planning in Sweden. The aim of the questionnaire was to investigate which instruments are the most interesting to study, and what kind of effects are the most important ones to study. The respondents rate the level of importance of each instrument/effect based on a 5-Likert scale ${ }^{20}$ and an option "Don't know"; if the question is not relevant for the respondent. The Likert scales used in this study are from level 1 to 5 . The questionnaire had two parts.

1. Importance of policy instruments: in the first part, we provided a set of potential instruments that can be studied using a simulation model. The categories of the potential instruments are obtained from a study that has been done on the policy instruments for urban transport ${ }^{14}$. However, the focus of that study was on soft policy instruments, where we exclude infrastructure investments and network expansion due to the recent shift of the focus of urban transport system planning from building new infrastructure to more efficient use of the existing infrastructure. But in the current survey, we decided to include infrastructure investment as well, since we wanted to see if there is still some interest and need for infrastructure investment among urban transport planners. The instruments are divided into four main categories: economic, administrative, informative, and infrastructure provision. The structure of the soft policy instruments is illustrated in Fig. 2. We asked the participants to rate the importance of studying each instrument.

2. Effects of policy instruments: the second part of the questionnaire was designed to understand the importance of the possible effects of instruments. We provided a list of possible effects and the participants were expected to rate which effect is more interesting for them to study. The possible effects of the instruments were effects on: emissions, route choice, parking choice, modal choice, vehicle occupancy level, road congestion, travel time, departure time of travellers, travel cost for passengers, and transport providers' revenue.

\subsubsection{Results of the survey}

We analysed the results in order to investigate what type of instruments and effects are the most interesting to study in the simulation model. The method used for analysis of the responses of the questionnaires was one-way ANOVA (Analysis of Variance) and post-hoc comparisons to understand if there is any significant difference between importance of different effects and in the same way, between different instruments. The result of one-way ANOVA indicated that there is a significant difference between importance of effects of policy instruments at $\mathrm{p}<.05$ level 


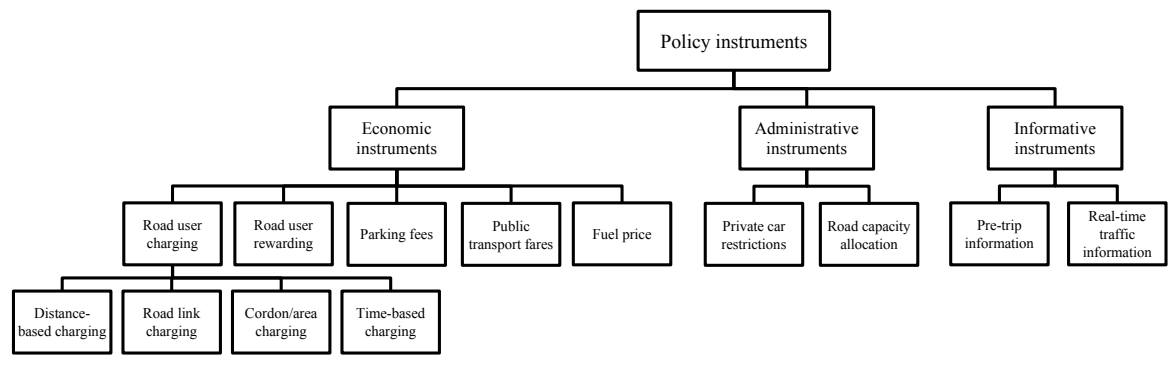

Figure 2: Categorization of the policy instruments used in the survey ${ }^{14}$

of significance $\mathrm{F}(9,198)=6.50, \mathrm{p}<.05$. Post-hoc pairwise comparisons using Fisher LSD correction revealed that there are some effects that are considerably less important than others, e.g. effects on: departure time, parking choice, travel cost for passengers, transport providers' revenue, and vehicle occupancy level. However, there are some effects that are more important than others for policy makers to know, e.g. effects on: modal choice, road congestion, route choice, travel time, and emissions.

In the same way, the result of one-way ANOVA indicated that there is a significant difference between importance of policy instruments at $\mathrm{p}<.05$ level of significance $\mathrm{F}(26,507)=3.56, \mathrm{p}$ « .05 . Post-hoc pairwise comparisons using Fisher LSD correction revealed that some policy instruments are significantly more important than others for policy makers to investigate the effects of. The instruments that are more important are: parking fees, public transport fares, road user charging, road infrastructure, fuel prices, railway tracks, public transport fares, public transport routing, etc. The instruments that seem to be less important are: Public awareness campaigns, flexible working hours, bike sharing systems, parking guidance information systems, pre-trip planning information systems, speed limit, etc. Some instruments are in between, such as convenience, bike parking places, and bike lanes.

Fig. 3 illustrates the most important findings from our survey on importance of policy instruments while Fig. 4 shows the effect of each policy instrument from respondents' point of view. The results of the survey show that instruments like fuel prices, public transport fares, and road infrastructure are more important than soft instruments such as different kinds of information systems, and public awareness campaigns. We have used these results in choosing relevant scenarios in our empirical study that is explained later.

\subsection{Scope of the simulation}

The scope of the simulation is defined as commuters between two important cities in south of Sweden: Malmö and Lund. 1) Malmö is the third largest city in Sweden with a population of above 300,000. More than 700,000 people are living in the Malmö metropolitan area. 2) Lund is a city in the province of Scania with 90,000 inhabitants that also belongs to Malmö metropolitan area. Lund is well-known within education, and Lund University is one of the Scandinavia's largest institutions in education and research. Lund is also a center for many high-tech industries. Due to the economic and educational importance of Malmö and Lund, many people commute everyday between these two cities which are located in the distance of about $20 \mathrm{Km}$ from each other. There are several transportation modes available for commuters: 1) train, 2) bus, 3) car, 4) bike. According to the recent survey around $44 \%$ of commuters use public transport, $55 \%$ car, and $0.03 \%$ bike or walk between Lund and Malmö. The scope of study is defined as people who live in the Lund area and study/work in Malmö. 
Instruments

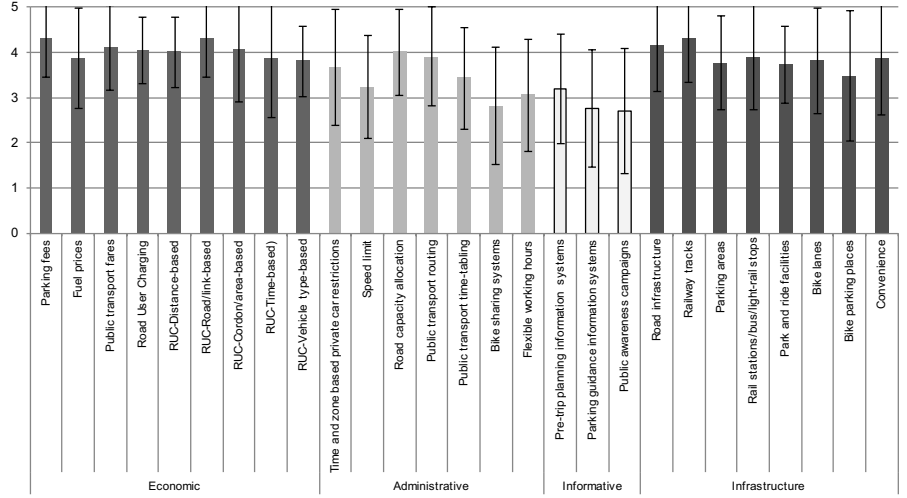

Figure 3: Results of the survey on comparison between policy instruments

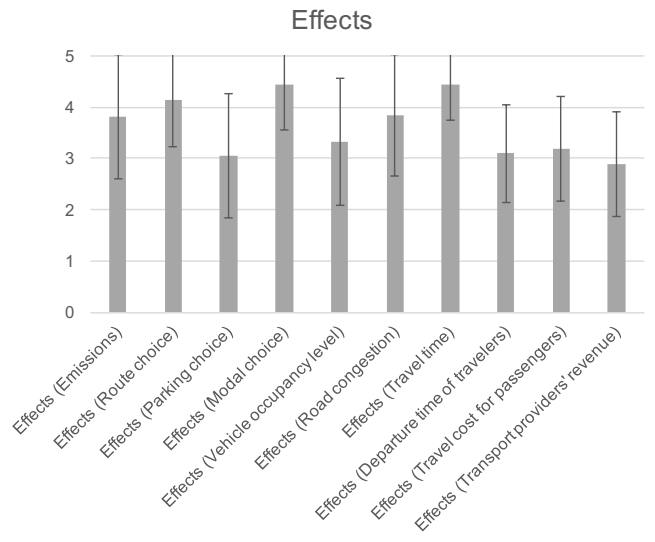

Figure 4: Results of the survey on effect of policy instruments

\subsection{Data preparation}

In order to generate a realistic sample of population for our travel behavior model, we have used data from RVU (The Swedish National Travel Survey) $^{\mathrm{c}}$ for the area of Scania county. The travel diary survey has been done in 2013 where the inhabitants of Scania county aged between 15-84 have been asked to provide their background information (e.g. age, income, access to car and bike), some personal preferences (e.g., which type of public transport card they

c https://www.trafa.se 
usually use, or how often they use public transport), and their complete travel diary including the travel purpose, the sequence of travel modes, the start and end points of the travel, the time the travel starts and ends, and the whole travel time. The survey has been distributed both through web and conventional post.

The number of target population who are qualified according to the criteria above is $1,013,926$, i.e. people who are registered in the Scania county and are between 15-84 years old at the time of the survey. The process of data gathering for this survey has been done during 5 weeks period in October and November 2013. However, travel data of a single person is just monitored for a single day during the sampling time. In this way, the whole sampling could be done in just one day, but instead data collection has been spread over 5 weeks in order to minimize the effects of special circumstances (e.g. in case a special public transport campaign is introduced in a day). Furthermore, in this way, both weekdays and weekends could be included. The exact date that the travel survey has been reported for is identifiable in the survey.

According to statistics mentioned in the RVU report ${ }^{22}$, the response rate is generally higher amount women than men. Also, the elderly has responded more than younger generation. Response rate among married and widowers is greater than unmarried people. Furthermore, people born in Sweden have generally a higher response rate compared to people born abroad, with the exception of the area Lomma-Bjarred in which the response rate is higher among those born abroad. This means the respondents are over representing the old Sweden-born married/widower women. However, this can be compensated using a weighting method for decreasing the effect of difference between demography of the sample in the RVU report compared to the real population.

According to the scope of our empirical study, we have filtered out some records that were not relevant. Since the scope of the study is defined as people who live in the Lund area and study/work in Malmö, we included the work/study travels, and excluded the other travels that have other travel purposes (e.g. shopping, leisure, dropping off and taking children at/from school). The filtering is done in two steps as follows.

1. Filtering based on travel purpose: in the first step, all the records that have other travel purposes than work or study are excluded from the list. The travel purpose varies among different options such as work, school/study, business trip, collect/leave children at school, shopping, healthcare/authority/service, exercise/association, leisure/ family and friends, travel back to home, etc.

2. Travellers: the next step is to identify the travellers who live in the Lund area and work in Malmö. We used some of the variables in the RUV data to generate target traveller population. Variable "bostad_komkod", refers to the municipality code of the residence address. We have kept the records that are related to travellers live in one of the municipalities of Lund (1281), Staffanstorp (1230), Burlöv (1231). The variable "rf4_komkod", refers to the municipality code of the final destination. Since we have already filtered out other travel purposes, this field is either referring to the work or study place. We aimed to identify the travellers who work or study in Malmö; therefore, we filtered out all the other data except the data whose variable "rf4_komkod" is equal to the code for Malmö which is 1280 .

After these two steps, we had all the records for the travellers who travel for the study or work purposes from their own home in Lund area to the work or study place in Malmö. The number of travellers who match these criteria was 333 unique travellers from which we removed 20 travellers since the address of these 20 travellers was not recognized by our on-line travel planner API. In order to get the travels back home, we repeated the same procedure on the dataset. We have further double checked if the same travelers appear in both lists which means that the travellers are commuters between Lund and Malmö and not travelling to another destination afterwards. When we created the list of traveller agents, we filled in all the necessary data for ASIMUT model using the information available in RVU survey about each traveller's socio-demographic attributes. All attributes used in the ASIMUT model and the way that we extracted data for each variable is explained as follows.

1. Age: the age of people at the time of the survey (autumn 2013) exists in the database and copied without any change to the relevant variable in the ASIMUT database.

2. Gender: the gender of travellers exist in the database and copied without any change.

3. Income: the income value provided by RVU is the annual income level for the whole family. In order to estimate the individual income of the traveller, we needed to know how many persons over 18 live in the household. We assumed that there is a high chance that family members who are older than 18 might have income. We took the 
Table 2: Income level values of each traveler agent

\begin{tabular}{ccc}
\hline Income level code (RVU) & Household Income level (RVU) & Assumed household income value (ASIMUT) \\
\hline 1 & $<100000$ & 80000 \\
2 & $100001-200000$ & 150000 \\
3 & $200001-300000$ & 250000 \\
4 & $300001-400000$ & 350000 \\
5 & $400001-500000$ & 450000 \\
6 & $500001-600000$ & 550000 \\
7 & $600001-700000$ & 650000 \\
8 & $700001-800000$ & 750000 \\
9 & $>800001$ & 1000000 \\
\hline
\end{tabular}

number of people aged between 19 to 64 in the household from the RVU survey. The annual household income is first divided by 12 in order to get monthly income, then the result is divided by the number of people in the household aged between 19 to 64. For example, if we have a person whose annual household income is level 7 according to RVU data, and there are 2 persons between 19-64 in their household, we assume that the monthly income of the person is $(650000 / 12) / 2=27083$ Swedish Krona. The income levels are defined in 9 different levels, as showed in Table 2. Since for ASIMUT we need categorical data, the income level is defined in the range of 9 categories that is demonstrated in Table 2

4. Home address: in order to assign a home address to a person, we used a number of variables from RVU: "bostad_tatnamn" referring to the urban area name of the home, "rf1_Samsnamn" referring to the SAMS area of the start point of travel, "hem_buss" referring to the distance to the nearest bus station from home in meters, "hem_jvg" referring to the distance to the nearest train station from home in meters. First the urban area of the home in SAMS ${ }^{d}$ atlas is located, then the SAMS name in the SAMS atlas is found. At the next step, the boundaries of the SAMS area in Google Maps are located. According to the distance to nearest bus stop and nearest train station, we manually generated a home address that was the closest option based on the information we had about each traveller agent.

5. Work address: the process of generating work address is similar to the home address, except the fact that we used variables related to the final destination instead of origin. Since we have excluded all other types of travels, the final destination is definitely the work address of the traveller. The variables used in this case are: "rf4_Samsnamn" referring to the SAMS name of the destination point, "arb_buss" referring to the distance to the nearest bus station from work or study in meters, "arb_jvg" referring to the distance to the nearest train station from work or study in meters. To find a work/study places, we have considered the businesses/universities that were located in close vicinity of the final destination. Finally we have manually assigned a work/study place that matches the criteria mentioned above.

6. Work starting time: we used the variable "rf6_ankomsttid" which refers to the time of arrival of the trip.

7. Work end time: we have first generated another table for travels back to home where the variable "ärende_2" is equal to 10 , meaning that this is travel back to home. In this dataset, we have used the variable "rf2_starttid" as the end time of working.

8. Work flexibility: this field has been randomly assigned. There was no information about how flexible one's work is in the RVU dataset, and according to our knowledge there was no way to interpret any relevant information about it.

9. Mobility tool (public transport): is calculated according to some variables in the RVU data: "Koll" referring to if the person has a card that can use to travel on public transport or transport services. The responses are listed in 5 level, as showed in Table 3. We have also used variable "kollkort" that is the response of the travellers to the question "What kind of ticket do you use most often when traveling on public transport in Skåne?" The values of this variable show different types of tickets, e.g., student monthly card, special card for children, ticket bought

\footnotetext{
${ }^{\mathrm{d}}$ SAMS is a nationwide area division that was created in 1994 in cooperation with the municipalities. SAMS is based in the larger municipalities on the municipal subdivision.
} 
Table 3: Values of the variable "koll" in RVU, in response to if the person has access to public transport card

\begin{tabular}{cc}
\hline Koll code & Koll Value \\
\hline 1 & Yes, always \\
2 & Yes, most of the time \\
3 & Yes, sometimes \\
4 & No, rarely \\
5 & No, never \\
\hline
\end{tabular}

Table 4: Mapping values of the variable "cykel"to the ASIMUT database

\begin{tabular}{cccc}
\hline Cykel code & Cykel value (RVU) & Bike at home (ASIMUT) & Bike at work (ASIMUT) \\
\hline 1 & Yes, always & 1 & 1 \\
2 & Yes, most of the times & 1 & 0 \\
3 & Yes, sometimes & 1 & 0 \\
4 & No, rarely & 0 & 0 \\
5 & No, never & 0 & 0 \\
\hline
\end{tabular}

Table 5: Values of the variables in RUV dataset that are used to calculate Eco friendliness in ASIMUT database

\begin{tabular}{cc}
\hline Code & Value \\
\hline 1 & Car as driver \\
2 & Car as passenger \\
3 & Bus \\
4 & Train \\
5 & Scooter/motorbike \\
6 & Bike \\
7 & By foot \\
8 & Flight \\
9 & Taxi \\
10 & Färdtjänst (transportation service) \\
11 & Others \\
\hline
\end{tabular}

through SMS, etc. In our implementation of ASIMUT database the mobility tool attribute is assigned based on the responses of travellers to these two questions. For example, if the traveller has responded to the second question that she never uses public transport, the attribute value is "no", and if the traveller uses any type of the ticket, that ticket type is directly transferred to the ASIMUT database.

10. Availability of bike at home and work: we used the variable "cykel" from RVU database that refers to how often the traveller has access to bike. The responses value from RVU and the interpreted value in the ASIMUT are listed in Table 4.

11. Eco friendliness: the value of this attribute is interpreted from the fields "huvudfm", "rf5a_färds1", "rf5b_färds2", "rf5c_färds3", "rf5d_färds4", "rf5e_färds5" in RVU dataset. These data refer to the main mode of public transport that the person uses and also the sequence of all the transport modes that have been used in that travel. Table 5 shows the list of values for the variables above. In case the traveller uses car as main transport mode, the value of eco-friendliness is considered to be low. In case the traveller uses one of the public transport modes as the main travel mode, uses bike, or walks, the value is assumed to be high. In cases that the traveller has combined car with some public transport mode, biking or walking, the value is considered to be average. This value is later double checked with the traveller's answer to the question about "kollkort" which is mentioned before. In case the traveller never uses public transport, the traveller should have low eco-friendliness.

12. Usual parking fee: in the RVU dataset the attributes "park_arb_1" to "park_arb_9" are used to estimate parking fees of the traveller in our implementation of ASIMUT model. These attributes refer to the availability of free parking, or if the traveller has some kind of company car and does not need to pay the parking fee. These are 
Table 6: Values of the attributes in RVU dataset about parking options

\begin{tabular}{cc}
\hline Variable & Description \\
\hline park_arb_1 & Free parking \\
park_arb_2 & Parking with charge \\
park_arb_3 & Benefit-rate parking \\
park_arb_4 & Company car/car pool \\
park_arb_5 & Company car \\
park_arb_6 & Compensation for own car \\
park_arb_7 & Subsidized public \\
park_arb_8 & transport pass \\
park_arb_9 & None of the above options \\
\hline
\end{tabular}

9 different variables in the RVU dataset, but there are only few cases that more than one attribute is filled. The values of these attributes are represented in Table 6. In all cases except for "parking with charge", the amount of parking fee is considered to be 0 . In case of "parking with charge" option, the parking fee is considered to be 60 Swedish Krona.

13. Access to car: is generated based on three different attributes in the RVU dataset: 1) "körkort" referring to if the person has driving licence or not, 2) "bilar" referring to how many cars are available in the whole household, and 3) "bilvidbehov" referring to how is the availability of the car for the person in case she needs one on a scale from 1 to 5 (always, most of the time, sometimes, rarely, never). First of all, if the person has no driving licence and the availability of the car when needed is low, it is assumed that the person has no access to any car. In case the person has driving licence but the family has no car, and the availability of the car when needed is low, it is also assumed that the person has no access to the car (i.e. in ASIMUT database, 'have_car' is set to zero for that specific traveler). The same applies when the person has driving licence and family has more than one car, but accessibility to the car when needed is relatively low.

\subsection{Calibrating ASIMUT}

Before we can use the adopted ASIMUT model for solving transport scenarios, the model needs to be calibrated. Transportation simulation models are mathematical abstractions of transportation systems where output is calculated based on particular equations given a specific input data. In our case the input data consists of two main groups of data: 1) travellers' characteristics, 2) travel options. The main output of the simulation in our model is travel behavior that is defined by decisions that travellers take to choose a travel option. Calibration means adjusting the model's parameters in order to minimize the error referring to the difference of main travel mode of output of the simulation $\left(\right.$ Output $\left._{\text {sim }}\right)$ and observed actual travels in the real world (Output $\left.t_{\text {obs }}\right)$.

Since we did not have access to details of the real decisions of each individual (i.e. which train or bus line they take), we used an aggregated data on mode choice to calibrate the model. In the RVU database the travel mode choice of respondents is available for that particular day that the traveler has reported. We aggregated the bus and train as public transportation mode versus other travel modes such as driving car, cycling, or walking. We used the chosen travel mode (public transport or car) as the observed output for calibrating our simulation model. We generated 313 agents in the data preparation step, and for each traveller we generated two travels, one from home to work/study and one for the travel back to home. The simulation runs for 10 days in order to capture the effects of different weather conditions and different day types (i.e. weekend or working day). Therefore, the number of all travels reached to 6260. We applied genetic algorithm ${ }^{10}$ as a heuristic approach which is based on the survival of the fittest candidates to find as good as possible values for the parameters of the travel behavior model that minimizes the error between the output of the simulation and the observed output. We used the single arithmetic crossover method ${ }^{18}$ for our crossover function. We started our heuristic approach by defining an initial set of 10 candidates for our 6 parameters. For generation of the next set of parameter values as children, we divided them to two groups. For the first group, we used values of the best parent as input to the crossover function and for the second half of the children, the next best 
Table 7: Optimum values for the parameters of travel behaviour model

\begin{tabular}{llc}
\hline$\#$ & Parameters of the model & Optimum value \\
\hline 1 & Cost & 5.5 \\
2 & Duration & 2.2 \\
3 & Walking distance & 2.2 \\
4 & Cycling distance & 3.7 \\
5 & Number of changes & 2.1 \\
6 & $\mathrm{CO}_{2}$ emission & 0.4 \\
\hline
\end{tabular}

parent is used as input. In order to keep diversity to the next generation of values, we let the last child to be generated by mutation. Then we let the simulation run for couple of days until the error did not change significantly anymore. Using parameters from the best set of candidates, we reached the accuracy of $72 \%$ which means our model selected 4538 correct travel modes out of total 6260 travels. The winner set of parameters are represented in Table 7.

It should be noted that we calibrated the model according to the choice of mode of the travelers on one single day from RVU data. However, in ASIMUT we have included travels of a traveler agent over a period of 10 days in order to investigate the effect of weather and different timetables. We have used the same main mode of transport of traveler in the RVU survey for calibrating our model. The lack of travel information in the RVU survey for travelers in different days with various weather conditions and time tables, might be one source of error in calibration. On the other hand, the RVU survey has been done over a period of 5 weeks, so that a various weather condition has affected travel choices during this 5 weeks which is not recorded in the survey. Moreover, since the RVU survey is done some years ago, it does not refer to exactly the same travel options that we get from on-line services now. It might be the case that current public transport possibilities are extended compared to the time RVU survey has been done. This can also be considered as one source of error in calibration.

\subsection{Simulation scenarios}

Based on the survey results on priority of policy instrument in southern Sweden that we presented earlier, the most important concern of decision makers is to affect modal choice, road congestion, route choice, travel time, and $\mathrm{CO}_{2}$ emission through policy instruments such as fuel prices, public transport fares, and road infrastructure. Our empirical study aims at simulating effect of these three policies on commuters' behavior.

1. Increase fuel prices by $\mathbf{4 0 \%}$ : one of the main policies to decrease $\mathrm{CO}_{2}$ emission is to increase cost of using car that aims at encouraging people to choose public transport mode instead of personal cars. In this scenario we increased the fuel prices by $40 \%$ that leads to increasing the cost of using car by approximately $20 \%$. We ran the simulation to investigate the effect of this policy on $\mathrm{CO}_{2}$ emission, modal choice, and travel time.

2. Decrease public transport fares by $\mathbf{5 0 \%}$ : another policy to affect travellers' modal choice and accordingly $\mathrm{CO}_{2}$ emission is to decrease public transport fares to encourage people to choose public transport mode instead of personal cars. In this scenario we decreased the public transport fares by $50 \%$ and ran the simulation to investigate the effect of this policy on $\mathrm{CO}_{2}$ emission, modal choice, and travel time.

3. Build a new train track: adding new infrastructures to the transportation system can affect several aspects of travel behavior. In our empirical study, we added a new train station and a new route to the system. Since this train station and the route does not exist, the on-line travel planners cannot be used to produce the travel options. Therefore, we had to manually generate the travel options for this scenario by defining new time tables for trains. We used suggested time tables by a planned but stopped project (Simrishamnsbanan ${ }^{\mathrm{e}}$ ) on building a train link between malmö and Simrishamn. We assumed that the hypothetical train runs every half an hour from Dalby to Malmö and vice versa starting from 05:30 in the morning to 01:30 after midnight. Moreover, a one-way train ticket for a trip between Dalby and Malmö assumed to cost 50 SEK for a single passenger. The result of the simulation for adding a new train station/route in comparison with the baseline and other scenarios is illustrated

\footnotetext{
${ }^{\mathrm{e}}$ http://www.simrishamnsbanan.se/strackningen/tidtabell/
} 


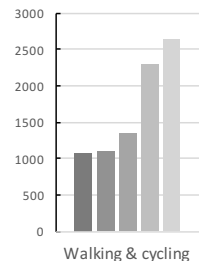

$(\mathrm{km})$

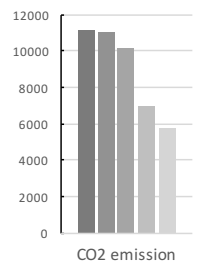

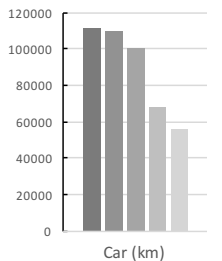

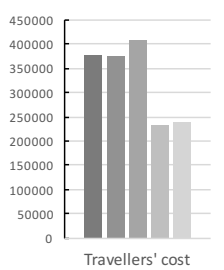

(SEK)

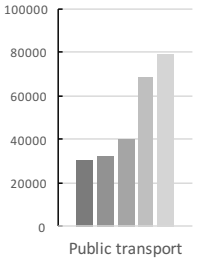

(km)

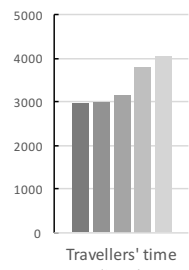

(hour)
- Baseline

- Scenario 1: Adding Dalby station

Scenario 2: fuel price

$+40 \%$

- Scenario 3: Public

trasportation fare $-50 \%$

Scenario 4: combination of 2,3

maseline

- Scenario 1: Adding

Dalby station

-1Mcenario 2: fuel price

$+40 \%$

InScenario 3: Public trasportation fare $-50 \%$

Scenario 4:

combination of 2, 3

Figure 5: Results of the simulation for the baseline state, adding a new train track between Dalby and Malmö (scenario 1), increasing the fuel price by $40 \%$ (scenario 2), decreasing public transport fares by $50 \%$ (scenario 3 ), and combination of the scenarios 2 and 3

in Fig. 5. In order to investigate the effect of different scenarios on travellers who live in Dalby and commute to Malmö we repeated the simulation for only 16 commuters living in Dalby.

\subsection{Simulation results}

The results of the simulation for three above-mentioned scenarios and combinations of them (Fig. 5 \& Fig. 6) seems to be rational. A comparison between share of the public transportation in the baseline condition, scenario 2, and scenario 3 shows that by increasing fuel price by $40 \%$, the public transport share increases by $29 \%$. The results also show that in scenario 3 , decreasing the public transport fare to $50 \%$ leads to increasing use of public transportation by $123 \%$. Consequently, combination of scenarios 2 and 3 results in increasing the use of public transportation by $158 \%$. However, adding a new train track in scenario 1 has an insignificant effect since only 16 travellers out of 313 live in Dalby and could potentially use the hypothetical train. Also a comparison between the traveller's time spent on commuting between Lund area and Malmö reveals that average time spent on commuting grows by using more public transportation that is also acceptable; however this growth is not significant. The average travel time in baseline condition is about 28.5 minutes for each travel; in scenario 2 the travel time reaches to 30 minutes; in scenario 3 it is 36.5 minutes; and in the combination of scenarios 2 and 3 the average travel time is 39 . This means that the speed of public transportation in southern Sweden is relatively close to driving car for commuting. The result of comparison between walking (\& cycling) and driving share in the total transportation is also acceptable. By increasing the share of public transport in scenario 2 and 3 and combination of 2 and 3, walking \& cycling share increases since travellers need to walk or cycle more towards bus or train stations to use public transportation. Moreover, increasing the use of public transportation in scenarios 2, 3, and combination of 2 and 3 leads to less driving cars between two cities and less $\mathrm{CO}_{2}$ emission. The analysis of travellers' cost of transportation shows that in scenario 2 by increasing use of public transportation due to the increasing fuel price by $40 \%, 9 \%$ of car drivers switched to public transportation that most probably decreases their travel cost, but still for $68 \%$ of the commuters who drive to work the fuel expense increases by $40 \%$. Since the portion of drivers who switch to public transport is significantly less than those who keep driving 

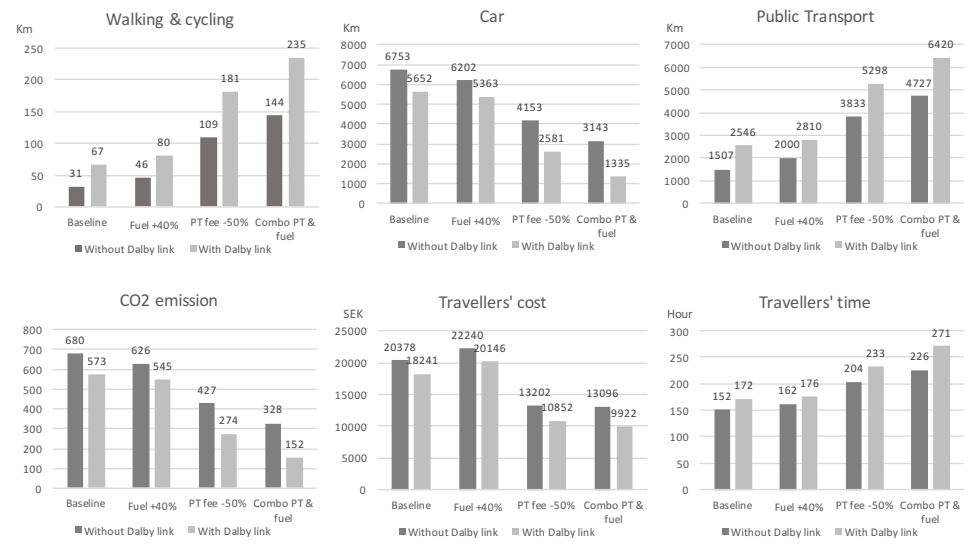

Figure 6: Results of the simulation only for passenger living in Dalby and commuting to Malmö before and after adding a new train track between Dalby and Malmö. The result includes the baseline state, increasing the fuel price by $40 \%$, decreasing public transport fares by $50 \%$, and combination of both scenarios

to work in scenario 2 , the average cost of travel in scenario 2 (65 SEK) increases compared to the baseline condition (60 SEK). While in scenario 3, by decreasing public transportation fare to half, $37 \%$ of the car drivers switch to a very cheap public transportation mode that decreases the average cost of each travel to 37 SEK in scenario 3 . Results of the simulation for only 16 travellers living in Dalby (Fig. 6) conforms to the results of the general simulation for all 313 travellers that shows the validity of our model for even hypothetical change in the infrastructure. Furthermore, by comparing results of the simulation for the the same population of Dalby before and after adding Dalby train (Fig. 6) we can see that more passengers living in Dalby use public transport when the train option is available. While before adding the train, the only possibility for using public transport for commuting was either taking an indirect bus through Lund or a combination of taking a bus to Lund and a train from Lund to Malmö.

\section{Conclusions \& Future Work}

Our empirical study revealed the great potential of using on-line travel planers in two of three simulation scenarios: scenario 2 (changing fuel prices) and scenario 3 (changing public transport fare) while preparing input data for scenario 1 (adding a new train station/route to the system) was cumbersome and time consuming. Therefore, we can conclude that using existing on-line travel planners for preparing input data is more efficient for scenarios where the infrastructure does not change otherwise the input data needs to be prepared manually. However, the existing APIs of the on-line travel planners could be extended in a way that provide possibility of adding hypothetical timetable of a new train or bus to the system. Another improvement that can be suggested to the providers of existing travel planners is to generate multi-modal travel options that includes all travel modes such as cycling. In this study, we merged services of a local travel planner with Google API to include cycling in combination with public transport as part of available travel options to the simulation. Finally, we recommend that on-line travel planners provide personalized travel options based on the priorities and preferences of individuals.

Results of this paper showed that using on-line travel planners to prepare input data for agent-based simulation can be a very efficient and a rather fast approach to simulate scenarios (like scenarios 2 and 3 in this paper) that do not require change in the infrastructure. Total time to run simulation for our first and second scenarios for 313 traveler agents traveling from home to work and vice versa during 10 days, i.e., in total 6260 , was about 4 minutes on a normal 
Mac book computer. However, to get the travel options from on-line travel planners such as Google Maps API in our case, we needs time, computational resources, and of course budget to pay for requesting a high amount of data. But when the data is collected, it can be reused to run different scenarios.

Reviewing previous studies on using computational models for analyzing the effect of transport policies on transportation systems shows that agent-based models have not been used so much in spite of their great potential for simulating dynamic aspects of policy instruments and travel behavior ${ }^{14}$. The main reason can be the need for a lot of data which is hard to prepare for the system. In this paper we showed how to use the results of a survey to generate a sample population of traveler agents and generate rest of the needed data for simulation using on-line travel planners. This approach can lead to more widespread use for agent-based simulation in solving more complex transportation scenarios.

ASIMUT can be further developed in order to include more factors influencing travel behavior, e.g. safety, comfort. This development needs more research on travel behavior modeling as well as high quality input data about the travelers. The model can benefit from using the new generation of travel surveys that are based on collecting data from mobile phones. Using a more detailed travel survey as input to the model, can increase the quality of the results and opens opportunities for investigating more complicated policy instruments due to availability of more detailed data about travelers and their travels.

\section{Acknowledgements}

We thank the National ITS Postgraduate School for their support. This research was done in collaboration with K2 - The Swedish Knowledge Centre for Public Transport.

\section{References}

1. S. Algers, L.-G. Mattsson, C. Rydergren, and B. Östlund. Sampers: erfarenheter och utvecklingsmöjligheter på kort och lång sikt. 2009.

2. S. An, J. Cui, and L. Li. Agent-based approach to model commuter behaviour's day-to-day dynamics under pre-trip information. Intelligent Transport Systems, IET, 5(1):70-79, 2011.

3. M. Balmer, M. Rieser, K. Meister, D. Charypar, N. Lefebvre, K. Nagel, and K. Axhausen. Matsim-t: Architecture and simulation times. Multi-agent systems for traffic and transportation engineering, pages 57-78, 2009.

4. D. Banister. The influence of habit formation on modal choice-a heuristic model. Transportation, 7(1):5-33, 1978.

5. M. Ben-Akiva and S. Lerman. Discrete Choice Analysis: Theory and Application to Travel Demand. MIT Press series in transportation studies. MIT Press, 1985.

6. E. Ben-Elia, R. Di Pace, G. N. Bifulco, and Y. Shiftan. The impact of travel information's accuracy on route-choice. Transportation Research Part C: Emerging Technologies, 26:146-159, 2013.

7. P. Bonsall. Traveller behavior: Decision-making in an unpredictable world. In Intelligent Transportation Systems, volume 8, pages 45-60. Taylor \& Francis, 2004.

8. J. Denrell and J. G. March. Adaptation as information restriction: The hot stove effect. Organization Science, 12(5):523-538, 2001.

9. A. Drogoul, E. Amouroux, P. Caillou, B. Gaudou, A. Grignard, N. Marilleau, P. Taillandier, M. Vavasseur, D.-A. Vo, and J.-D. Zucker. Gama: multilevel and complex environment for agent-based models and simulations. In Proceedings of the 2013 international conference on Autonomous agents and multi-agent systems, pages 1361-1362. International Foundation for Autonomous Agents and Multiagent Systems, 2013.

10. O. B. Espinosa. A genetic algorithm for the calibration of a micro-simulation model. arXiv preprint arXiv:1201.3456, 2012.

11. G. Gigerenzer, R. Selten, et al. Rethinking rationality. Bounded rationality: The adaptive toolbox, 1:12, 2001

12. N. Gilbert and K. Troitzsch. Simulation for the social scientist. McGraw-Hill Education (UK), 2005.

13. B. Hajinasab, P. Davidsson, J. Holmgren, and J. A. Persson. On the use of on-line services in transport simulation. Transportation Research Procedia, 21:208-215, 2017.

14. B. Hajinasab, P. Davidsson, and J. A. Persson. A survey on the use of computational models for ex ante analysis of urban transport policy instruments. Procedia Computer Science, 32:348-355, 2014.

15. B. Hajinasab, P. Davidsson, J. A. Persson, and J. Holmgren. Towards an agent-based model of passenger transportation. In International Workshop on Multi-Agent Systems and Agent-Based Simulation, pages 132-145. Springer, 2015.

16. B. Hajinasab, P. Davidsson, J. A. Persson, and J. Holmgren. Towards an agent-based model of passenger transportation. In Lecture Notes in Computer Science, Multi-Agent Based Simulation XVI, volume 9568, pages 132-145. Springer, 2015.

17. C. Hansen. Report on scenario, traffic forecast and analysis of traffic on the ten-t, taking into consideration the external dimension of the uniontrans-tools model version 2: Calibration and forecasts 2020 and 2030. Recent Developments in Transport Economics, 2009.

18. J. H. Holland. Adaptation in natural and artificial systems: an introductory analysis with applications to biology, control, and artificial intelligence. MIT press, 1992.

19. A. Horni, K. Nagel, and K. W. Axhausen. The multi-agent transport simulation MATSim. Ubiquity Press London, 2016. 
20. R. Likert, S. Roslow, and G. Murphy. A simple and reliable method of scoring the thurstone attitude scales. The Journal of Social Psychology, 5(2):228-238, 1934

21. T. Litman. What's it worth: Economic evaluation for transportation decision-making. In Internet Symposium on Benefit-Cost Analysis Transportation Association Of Canada. http://www. vtpi. org/worth. pdf, 2001.

22. C. W. M. U. Mats Nyfjäll, Pär Wikman. Resvaneunder

23. S. McDonnell and M. Zellner. Exploring the effectiveness of bus rapid transit a prototype agent-based model of commuting behavior. Transport Policy, 18(6):825-835, 2011.

24. J. N. Prashker and S. Bekhor. Route choice models used in the stochastic user equilibrium problem: a review. Transport reviews, 24(4):437-463,

25. L. Ramstedt. Transport policy analysis using multi-agent-based simulation. Number 2008: 09. 2008.

26. H. A. Simon. Models of bounded rationality: Empirically grounded economic reason, volume 3. MIT press, 1982.

27. T. Takama and J. Preston. Forecasting the effects of road user charge by stochastic agent-based modelling. Transportation Research Part A: Policy and Practice, 42(4):738-749, 2008.

28. D. Teodorovic. Transport modeling by multi-agent systems: a swarm intelligence approach. Transportation planning and Technology, 26(4):289$312,2003$.

29. N. Thomopoulos, M. Givoni, et al. ICT for transport: Opportunities and threats. Edward Elgar Publishing, 2015.

30. A. Tversky and D. Kahneman. Advances in prospect theory: Cumulative representation of uncertainty. Journal of Risk and uncertainty, 5(4):297323,1992 


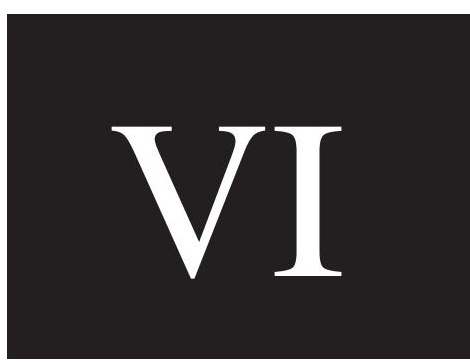





\title{
VISUALIZATION OF DATA FROM TRANSPORTATION SIMULATION SYSTEMS
}

Banafsheh Hajinasab, Department of Computer Science, Malmö University, SE-205 06, Malmö, Sweden, banafsheh.hajinasab@mah.se

Paul Davidsson, Department of Computer Science, Malmö University, SE-205 06, Malmö, Sweden, paul.davidsson@mah.se

Jonas Löwgren, MEDEA Collaborative Media Initiative, Malmö University, SE-205 06, Malmö, Sweden, Jonas.lowgren@mah.se

Jan. A. Persson, Department of Computer Science, Malmö University, SE-205 06, Malmö, Sweden, jan.a.persson@mah.se

\begin{abstract}
Information visualization, as a way of presenting different data types in an understandable form, has the potential to support the analysis of transportation data. Visualization can often help decision makers to efficiently analyse large amount of information. One application area of information visualization is to support the analysis of the transportation data and thus facilitate the decision-making process. Most of the previous studies in this area have focused on visualization of transportation infrastructures such as roads, bridges in order to enhance the public awareness regarding upcoming projects which makes it easier to reach a consensus on the high-level decisions. However, the main focus of this article is on methods for visualization of data generated by transportation simulation systems to support analysis of the consequences of applying different transport policy measures, such as the introduction of road user charging or investment in new infrastructure. In this work, we investigate how visualization techniques could address the challenges of transportation simulation data analysis in order to facilitate the decision-making process. For this purpose, we have applied the visualization methods to a real implemented agent-based transportation simulator called TAPAS. In this case study, we have analysed the visualization related requirements of users using a user-centric approach and the visualization tool has been designed and developed based on the identified requirements.
\end{abstract}

Keywords: Transportation simulation visualization, decision support system, policy impact assessment, logistics, transport planning, usability.

$13^{\text {th }}$ WCTR, July 15-18, 2013 - Rio de Janeiro, Brazil 
Visualization of data from transportation simulation systems

HAJINASAB, Banafsheh; DAVIDSSON, Paul; LÖWGREN, Jonas; PERSSON, Jan. A

\section{INTRODUCTION}

There is a growing use of simulation in transportation and there have been established national and international models which can predict future flows in freight transport network (De Jong and Ben-Akiva, 2007; Brunner et al, 1998). The main idea is resting in the attempt to simulate the real system characteristics in a computer system and observe how applying different scenarios will affect the behaviour of the transportation system (Holmgren et al, 2012a). Simulation has a potential to assist in planning processes where the decision makers wish to be aware of the consequences of a specific decision on the whole system (Fishburn et al, 1995). One of the key applications of simulation in the transportation context can be related to the cost reduction and the increase of the quality of service provided (Fishburn et al, 1995). However, not all the transportation simulation systems are necessarily successful regarding reducing costs and increasing quality. There are some factors which determine the degree of success of simulation models. One important factor is the "Ease of use" of the simulation system by different types of potential users (Brunner et al, 1998). Typically, a simulation system generates a large amount of data as output. This data often need to be processed and analysed in order to be useful. Hence, the way of analysing and presenting the output data to the stakeholders, can be a challenging task. As individuals acquire more information through their sense of vision than through all the other senses (Ware, 2012; Chen, 2004), visualizing the output data of transportation simulation systems could help to overcome the complexity of data presentation and analysis.

This research seeks to investigate how visualization techniques can address the challenges of transportation simulation data analysis in order to facilitate the decision-making process for transportation simulation users. To achieve this goal, the paper explores a multi-agent based simulation model for transportation and production called TAPAS (Holmgren et al, 2012) (Transportation And Production Agent-based Simulator). TAPAS is a micro-level simulation tool which aims to reveal the effects of applying different measures including governmental policies like taxes, and infrastructure investments and business strategies (Ramstedt et al, 2007). It can also act as a decision support system for public authorities and policy makers by predicting what the effects are when applying a specific set of production and transportation measures and whether it contributes to the government goals of sustainable economic development (Bergkvist et al, 2005). Furthermore, TAPAS can assist enterprises to make both operational and strategic decisions, for example, decisions regarding consignment size and storage locations and similar (Davidsson et al, 2008).

The process for designing the visualization tool is based on the "Systems Engineering" process (Wymore, 1993). The study followed the "System Development Life Cycle (SDLC)" as a life cycle of system development; in which there are Analysis, Design, Implementation, Testing and Evaluation phases (Post and Anderson, 2002). Furthermore, the "Spiral Model" is used for the migration from requirements to design solution (Boehm, 1988). This selection is made due to the fact that prototypes of the system are created so as to obtain user feedback during the development process. This method has an iterative nature and each iteration is repeated until the final product is made. Before implementing the visualization options, sketches for the proposed system are prepared. These sketches are presented to the users to obtain the approval and their feedback on design alternatives and further 


\section{Visualization of data from transportation simulation systems HAJINASAB, Banafsheh; DAVIDSSON, Paul; LÖWGREN, Jonas; PERSSON, Jan. A}

options. This may be seen as a type of an iterative process that is continued until a positive feedback is received from the users.

The rest of this paper is organized as follows. Section 2 introduces a literature review on information visualization. In section 3 we describe the core of our contribution by introducing the TAPAS visualization tool and its key design features and functionalities. Section 4 represents the evaluation of the proposed visualization tool. Section 5 concludes the paper by presenting our findings.

\section{RELATED WORK}

\subsection{Information Visualization}

It is crucial to understand the concept of visualization in order to develop a usable interactive tool for data visualization. Information Visualization is a relatively new area which is increasingly acquiring more and more attention in both academic and industrial environments. Here we explore the use of computer-supported interactive graphical representations for explaining data and amplifying cognition.

Visualization plays an important role in human understanding by providing visual representations of large amounts of data. This is required in order for data to be more usable and understandable for users (Ware, 2012; Mazza, 2009). "Visualizations have a small but crucial and expanding role in cognitive systems. Visual displays provide the highest bandwidth channel from the computer to the human" (Ware, 2012). Owing to the importance of visualization in real world problem domains, it has been growing tremendously in different contexts (Card et al, 1999; Chen, 2004). Obviously, the nature of the data to be visualized is different in various application areas. The choice of selecting a set of visualization techniques and applying them to a data set is highly dependent to the context of the specific data set. As an example, in case of existing inheritance relations between the data elements, graphs can be used as a way of representing and visualizing information (Herman et al, 2000). Layout algorithms have emerged as a way to solve the problems of visualizing complex and large data displayed in a graph. Some of these algorithms are focused on aesthetics criteria in the context of Graph Drawing (Di Battista et al, 1998; Kaufmann and Wagner, 2001), while others are based on drawing conventions and constraints (Di Battista et al, 1998). In addition, other types of visualization techniques, such as displaying data through diagrams are also available (Brasseur, 2006). Given the nature of the data and the type of analysis that should be drawn from the data, a mix of these approaches and techniques can be applied to reach usability requirements of potential users of the specific context.

Furthermore, visual attributes should be utilized to display data elements in different colours and shapes in order to increase readability of a visualized graph. Colin Ware (Ware, 2012) describes an ability of human cognitive system called "Pre-attentive processing" in his work. He defines the concept of pre-attentive processing as a theoretical mechanism which causes some aspects of a visual model more highlighted or distracted. Ware argues that there are some visual attributes which can be pre-attentively processed in the human vision system. Examples of these attributes are colour, spatial position, movement and form. The discussion

$13^{\text {th }}$ WCTR, July 15-18, $2013-$ Rio de Janeiro, Brazil 


\section{Visualization of data from transportation simulation systems HAJINASAB, Banafsheh; DAVIDSSON, Paul; LÖWGREN, Jonas; PERSSON, Jan. A}

above emphasizes the pre-attentive visual attributes as crucial assets of a visualization model.

This research focuses on visualization of data in the context of transportation. For visualizing a transportation network, special techniques for graph drawings are required. An important characteristic of transportation context is positioning the elements on the screen. Thus, the aim here is to investigate the techniques for visualizing a set of nodes and their relations.

There are some general approaches to positioning nodes and edges. The most important and commonly used approaches are listed below (Aris, 2008; Shneiderman and Aris, 2006):

- Force-base drawing algorithms: In this layout algorithm, the network of nodes and edges are simulated as an electrical system. There are attraction and repulsion forces between nodes and edges. These forces will apply to nodes and the position of nodes will change accordingly. The goal of this algorithm is to reduce the energy associated to graph layout. This algorithm has an iterative nature and it will be repeated until the energy system reaches a balanced state (Eades, 1984).

- Geographical approach: In this strategy, position of nodes is based on geographical attribute of nodes on a map (Becker et al, 1995).

- Circular topology: This layout algorithm tries to put nodes on a circle, so the edge crossings will be at the centre of the circle (Breitkreut et al, 2003).

The selection of which approach should be taken depends largely on the domain of data to be visualized. In this article, we rely on the Geographical approach as it meets the requirements of users in the transportation simulation context, where the position of the nodes should be as close as possible to their real positions on the map.

\subsection{Transportation system visualization}

Several research groups have targeted the transportation visualization field with different approaches. However, very little research is conducted on applying visualization methods to transportation simulation in order to support the analysis of simulation output data. The "Visualization in Transportation Committee", a sub-committee of the "Transportation Research Board" (TRB), focuses on visualization techniques for transportation issues. The majority of previous studies both in TRB and other research groups revolve around visualization of the physical infrastructures (e.g. a new road or bridge) and the impact that these infrastructure investments have on the transportation system. However, the emphasis of this article is on visualization of output data from transportation simulation systems, where the effects of applying transport policy measures on the whole transportation network are potentially predicted.

According to Hixson (Hixson, 2006), the main uses of visualization rest in the design process where the aim is to facilitate the work of designers and in obtaining the public approval to reduce the rework costs. It can also act as a complementary way in ensuring alignment of achievements with planned improvements (Richter et al, 2003). Although the approach of using visualization in transportation for design purposes is also a goal of TAPAS, this approach is relatively different. In the above direction, the use of visualization in the design

$$
13^{\text {th }} \text { WCTR, July 15-18, } 2013-\text { Rio de Janeiro, Brazil }
$$




\section{Visualization of data from transportation simulation systems HAJINASAB, Banafsheh; DAVIDSSON, Paul; LÖWGREN, Jonas; PERSSON, Jan. A}

process is limited to showing the anticipated final product (e.g. a road or a bridge) with respect to architectural factors as the input. By contrast, the aim here is to visualize the impact of applying different measures in transportation process, such as taxes.

Although visualization has been used in transportation field for many years and several studies have been done for improving and customizing visualization techniques for transportation goals, there is still a significant lack of research directed at application of visualization during the planning process (Hughes, 2008). Use of visualization in a planning process takes into account human and environmental aspects of transportation projects as well as the economic impact factors. Hughes believes that the research conducted so far in this field, mainly concerns the involvement of stakeholders in the design process and investigation of effective ways to demonstrate design alternatives to different stakeholders. Hughes argues that there is a need to apply visualization techniques in transportation by considering environmental perspectives in the planning process (Hughes, 2008).

Hughes also reviews current trends and approaches in visualization of the transportation data and argues that two techniques of visualization are mainly used in the context of transportation (Hughes, 2010):

- Map-based visualization: the nature of data in the context of transportation often is based on geographical information where using map as a background and visualizing transportation data on a map can be considered as a promising design solution. Typically, the transportation-related information consists of source, destination and routes between them. Thus, based on the characteristics of the transportation data, it appears to be more meaningful to rely on maps for visualization of freight data. An example of map-based visualization is illustrated in Figure 1 below.

- Using "Score Cards" and "Dash boards": These techniques can be used with the aim of tracking Key Performance Indicators (KPIs) of a system and also system level variables for users.

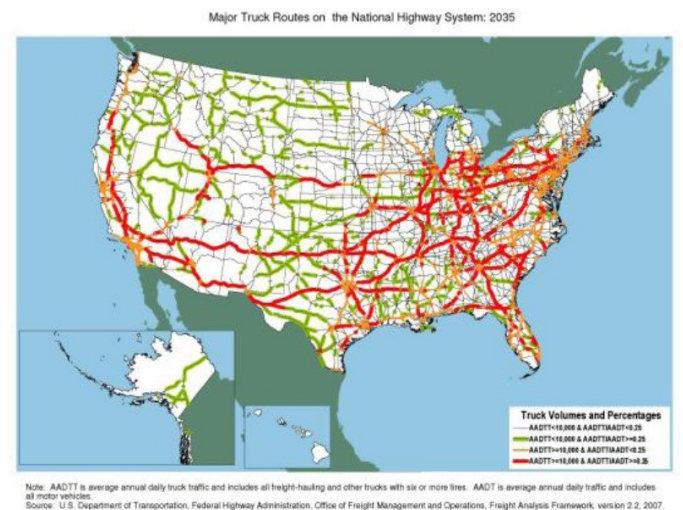

Figure 1 - A sample map-based visualization of freight data (Hughes, 2010)

$13^{\text {th }}$ WCTR, July 15-18, 2013 - Rio de Janeiro, Brazil 


\section{Visualization of data from transportation simulation systems HAJINASAB, Banafsheh; DAVIDSSON, Paul; LÖWGREN, Jonas; PERSSON, Jan. A}

P.J. Hoen, et al have conducted a research into developing a software system for visualizing a multi-agent model of logistics management (Hoen et al, 2004). Their proposed software features consist of visualizing the simulation process, routes, the appearing of loads and truck movements. The focus of the software is on the visualization of how the simulation system works (e.g. illustration of object movements) rather than visualization of the output results of the simulation. Therefore, in this paper we try to study the methods for visualization of the aggregated results of the simulation as opposed to the previous approach to visualize the events when they occur during the simulation. As the scope of this article is investigating of visualization techniques for a multi-agent based transportation simulator, where monitoring the effects of applying measures is the goal of simulator, it is very different form the research which conducted in this field so far.

\section{TAPAS CASE}

TAPAS is a Multi-Agent-Based Simulation (MABS) model of a transportation and production system including the operational and logistical decision simulators. TAPAS can be used to predict the consequences of various measures for decision and policy makers, before making any decision. Some of these measures are listed below (Bergkvist et al, 2005; Ramstedt et al, 2007; Holmgren et al, 2007):

- Governmental control policies, such as taxes and fees,

- Infrastructure investments, such as investments in a new road or railway,

- Strategic business related strategies, such as changing the location of a factory

Each TAPAS simulation scenario studies the effects of a certain set of measures on the supply chain. The output of TAPAS shows how supply chain actors are assumed to react to the above measures. Thus, the users of TAPAS can interpret the results and make more informed decisions and consequently avoid undesired effects. The main outputs of TAPAS simulation are described below (Davidsson et al, 2008; Holmgren et al, 2007):

- Economic effects, such as costs for fuel and taxes

- Logistic effects, such as route and mode choices

- Environmental effects, such as different types of emissions

The selection of TAPAS as the study case is made due to the fact that TAPAS is an implemented transportation simulation system without any visualization. Thus, it is possible to study how difficult and time-consuming the interpretation of the results can be without visualization especially for non-expert users. The idea is to explore the obstacles such users 


\section{Visualization of data from transportation simulation systems HAJINASAB, Banafsheh; DAVIDSSON, Paul; LÖWGREN, Jonas; PERSSON, Jan. A}

face in interpreting the results of simulation, and how the visualization can help overcome the challenges of data analysis.

There are some terms often used in the TAPAS documentation, and therefore in this research as for example (Holmgren et al, 2012a):

- Node: A node represents different kinds of actors in the transportation chain, for example: customer, producer (a factory), or a connection point (terminal)

- Transportation mode: A transportation mode indicates the type of transportation (e.g. road, rail, or sea)

- Link: A link is a directed connection between two nodes. A link can have exactly one mode of transportation. However, several links between two nodes which differ in their transport mode can exist.

\subsection{Requirements analysis}

This section examines the requirements of users who want to view the results from a transportation system simulator in a visual representation. The first step seeks to understand the problems users experience regarding readability and usability of TAPAS output, and how a visualization tool could help them. In addition, we need to know the type of target groups which potentially can use TAPAS, because their needs are considered in the design process of the visualization tool of TAPAS. This can be achieved through conducting interviews with TAPAS users who are mostly expert in the field of transportation.

TAPAS can have two types of users. The first group relates to public authorities, which are interested in predicting the impact of their decisions whilst fulfilling the governmental goals, e.g. emission targets. The second group concerns the private companies and other stakeholders of transportation and production, which wish to enhance their profit. As a result, different type of users may intend to apply different measures. For example, public authorities are more interested in the first group of measures (i.e. governmental control policies), while enterprises are more eager to predict the effects of their business related decisions (i.e. changing the location of a factory) for profit related purposes. However, enterprises might be also interested in monitoring the effects of a new transport policy (Holmgren et al, 2012).

For purposes of this research, in addition to the previous user groups, transportation simulation experts (e.g. developers of TAPAS) are considered as users. They can also be seen as TAPAS users, because of the fact that they enter various scenarios to the system and run simulation several times to generate simulation results.

Hence, we can categorize TAPAS users from a different perspective:

- Expert users, usually the analysts and researchers which run the simulation system and analyse the simulation results.

- End users, usually decision makers in both public authorities and companies.

$13^{\text {th }}$ WCTR, July 15-18, $2013-$ Rio de Janeiro, Brazil 


\section{Visualization of data from transportation simulation systems HAJINASAB, Banafsheh; DAVIDSSON, Paul; LÖWGREN, Jonas; PERSSON, Jan. A}

Each type of users has their own concerns and expectations from the system. In order to identify specific user needs, interviews are conducted with expert users of TAPAS. The interviews aim to define the user requirements and discover probable problems which TAPAS users face without visualizing the results. The questions of the interview are listed below:

1. What is the specification of TAPAS output? Which data is important to visualize?

2. How do you get output data from TAPAS? Is there currently any visualized output or not?

3. What are the major problems in the process of monitoring the results of simulation which cause difficulties and interrupts your work?

4. How much time do you spend as an outcome of above problems?

5. Can visualization help you in understanding the results? If yes, what is your suggestion for design of a visualization model?

Furthermore, two web-based questionnaires for potential end users are used in order to understand their special needs and interests with respect to the transportation simulation visualizer. The aim of the first questionnaire was to demonstrate the idea and some initial mock-ups of the tool to the end users. The second questionnaire was sent to 12 potential end users, after a short demo of the semi-final version of the tool.

The interviews with expert users reveal that a major problem for TAPAS users without a visualization tool is that the whole process of designing visible outputs from TAPAS is quite time-consuming. The interviewees agree that visualization can help TAPAS users to have a better and clearer understanding of the results. They had also some suggestions for visualizing. Full details of the expert users' answers to the interview questions can be found in (Hajinasab, 2011). Based on the interests of users regarding visualization of output, the important outputs to be visualized can be categorized as below:

1. Route choices (e.g. percentage of different routes)

2. Mode choices (e.g. percentage of different modes)

3. Transportation per link (e.g. number of vehicles per link)

4. Amount of emissions per route (e.g. $\left.\mathrm{CO}_{2}\right)$

5. Transport cost (per route)

The interview study further suggests for a need that visualization in TAPAS facilitate the process of analysing simulation results. Furthermore, the interview provides an initial idea regarding the requirements of the users with respect to visualization of output.

In order to find out which type of simulation data the end users are interested in, we used the knowledge extracted from the previous studies and the interview with expert users to suggest

$$
13^{\text {th }} \text { WCTR, July 15-18, } 2013 \text { - Rio de Janeiro, Brazil }
$$




\section{Visualization of data from transportation simulation systems HAJINASAB, Banafsheh; DAVIDSSON, Paul; LÖWGREN, Jonas; PERSSON, Jan. A}

possible data which they may be interested in. Sample examples and mock-ups from our design solutions are used in order to obtain the interviewees' opinion regarding their preferences for the information of simulation and how the final visualization tool should look like. The aim of these questions is to gain the users' opinions on the importance of showing information related to each route or each link in the transportation network. The respondents appear interested to have information related to each route.

As a conclusion of the user studies, the requirements of users can be categorised as follows:

- Req1. Showing input and output of simulation: We can conclude from the interviews that both expert and end users are interested in a visualization tool and they believe such system could facilitate their work. Also, they prefer to see which input (transport policy measure) results in a specific output; just visualization of simulation output is not deemed meaningful for them. Thus, we conclude that visualization of the input related to each output is also important for users.

- Req2. Showing different scenarios concurrently: Both types of interviewees would like to be able to see several different scenarios at the same time. They found this to be useful when comparing various scenarios and their related output. They also believe that this feature can help them in evaluating the output results and facilitate the decision making process.

- Req3. Showing simulation information based on links and routes: Furthermore, interviewees had some suggestions for which output is more meaningful to be visualized and which not. We can also conclude that they need to see information related to both links and routes.

\subsection{Design Features and Functionalities of TAPAS Visualization Tool}

Due to the fact that the design decisions could not be discussed with users just by explaining theories, we have designed several mock-ups of the visualization tool. A mock-up helps users imagine the real system and facilitate the process of designing functions and features of the visualization tool. Thereby, the mock-ups are designed in several steps based on the spiral method in order to obtain the feedback from expert and end users. Some of the initial results of the tool are presented in (Hajinasab et al, 2012)

Based on the user studies, the proposed User Interface of the TAPAS visualization tool can have two Tab Views; Link-based view and Route-based view. A simple sketch is produced as the first step. The relevant information of each category can be displayed in their related tab. During the discussion with users and by presenting the mock-ups, we could make valuable design decisions, such as the way of demonstrating different transportation modes. For displaying the route choice, the best solution based on previous work and user feedbacks is via the use of a graph. To put it simply, the proposed solution displays each origin and destination as a node of the transportation graph. For illustrating the percentage of using each route (e.g. the amount of ton/route), the solution could be drawing edges between nodes with different thicknesses. As a result of this visualization feature, the user of TAPAS can easily discover which route is used more frequently than others.

$13^{\text {th }}$ WCTR, July 15-18, $2013-$ Rio de Janeiro, Brazil 
Visualization of data from transportation simulation systems HAJINASAB, Banafsheh; DAVIDSSON, Paul; LÖWGREN, Jonas; PERSSON, Jan. A

As the next step, based on these main features of visualization system, we designed a more complete sketch. We also used this sketch for preparing the end-users' questionnaire.

In order to provide the user with an ability to make a comparison between different scenarios, the user can select a specific scenario at first when the program runs; following which the information related to this specific scenario is shown.

In the light of user requirements, we propose to visualize information of each main output (Cost, $\mathrm{CO}_{2}$, Transport work) and allow users to select the desired information from the right side bar in the Figure 2. In link-based view, the cost (or $\mathrm{CO}_{2}$, load) information for each link is shown above each link. In addition, different edge styles are used to exemplify the various transport modes of links, while, the cost (or $\mathrm{CO}_{2}$, load) information of each route is illustrated in route-based view. We can also, highlight the selected route. We have a graph in the right bottom corner of the Figure 2, which shows and compares the cost related to all routes. A set of final mock-ups of the proposed design is shown in Figure 2.

In general, main functionalities of the system, regarding user requirements and based on the previous studies in information visualization are designed as below:

- Show as a graph: Using colour, edge thickness, edge styles to differentiate links and routes; to show the simulation results.

- Draw charts: to illustrate data and make comparison between different scenarios.

- Link and route-based information on Graph: Showing information of cost, emission and amount of transport work on each link and route.

- Interactivity: The interactivity of the visualization toolkit empowers users to explore implications of different simulation scenarios and engages them in what-if- reasoning.

- Add a map as background: to increase usability and readability of the transportation data.

$13^{\text {th }}$ WCTR, July 15-18, 2013 - Rio de Janeiro, Brazil 


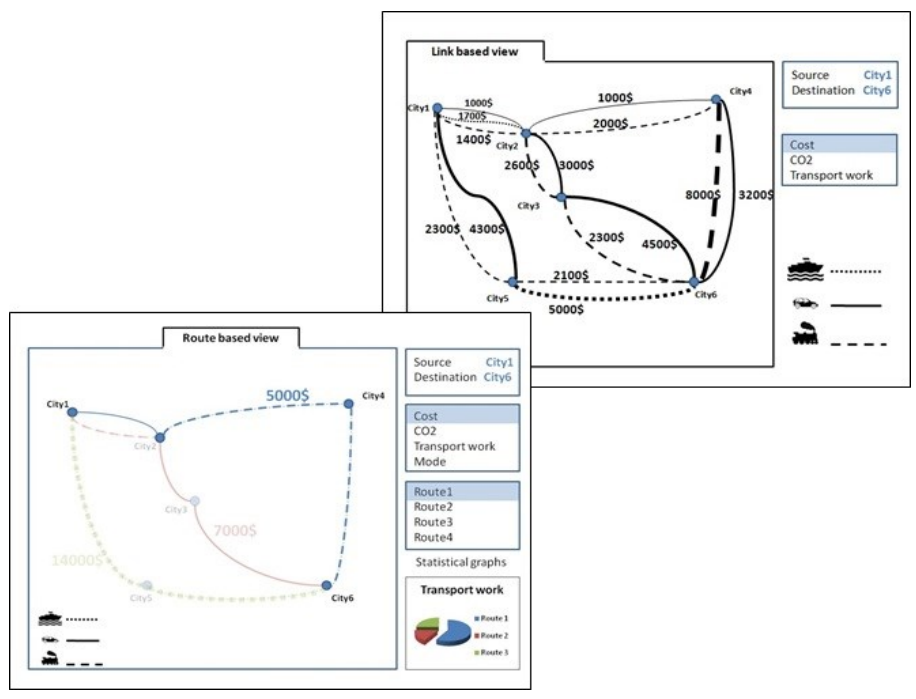

Figure 2 - Final mock-ups of the link-based and route-based view in the proposed design of the interface

\subsection{Development of visualization module}

TAPAS is implemented using the Java language, so it would be more integrated to use the same language for developing this tool. We have used Swing technology as an Application Programming Interface (API) for designing the user interface.

Another option would be to develop the tool on the top of the Google Maps API. The advantage of Google Maps API is that it can locate nodes on their spatial locations automatically so that we do not need extra classes and relations with graph drawing libraries to place nodes. However, the main issue is that we cannot use real positions in a real map for this tool. Because in some cases the positions of two nodes are very far from each other while some others are relatively close to each other, so if we use real coordinates in a real map it will decrease readability of the graph to a high extent. Thus, we need to customize the map and adjust the positions of the nodes either manually or automatically in order to increase usability and readability of the transport graph. Another problem of using Google maps API is that it contains too much information. This in turn makes it difficult for users to see the simulation results on the map. Furthermore, there are some limitations in using Google Maps API for this tool. For example, we need to use other classes and libraries to draw charts and other parts of the tool, which is not possible by using Google Maps API.

For implementing the graph drawing part of the tool, the JUNG library ${ }^{1}$ is regarded as the most suitable solution. JUNG library, Java Universal Network/Graph Framework, is a software library that provides a common and extendible language for the modelling, analysis,

\footnotetext{
1 “JUNG - Java Universal Network/Graph Framework” Internet : http://jung.sourceforge.net/
}

$$
13^{\text {th }} \text { WCTR, July 15-18, } 2013 \text { - Rio de Janeiro, Brazil }
$$




\section{Visualization of data from transportation simulation systems HAJINASAB, Banafsheh; DAVIDSSON, Paul; LÖWGREN, Jonas; PERSSON, Jan. A}

and visualization of data that can be represented as a graph or network. This selection is motivated due to the fact that JUNG is well-documented, easy to use, and well-supported.

A part of the visualization tool, consists of drawing charts and diagrams which has been implemented using the JFreeChart library.

Both input and output (i.e. simulation results) from TAPAS sit in a database. In order to access this database and retrieve desired data from the database, we used PostgreSQL and SQL queries. To explore the database, we have used pgAdmin III, which is a PostgreSQL administration and management tool.

The procedure of developing the functionalities of the visualization module is described as follows:

In order to show data in a graph, we use JUNG library and we draw the graph based on the information from the database. Several queries have been designed to obtain the desired data and draw the graph based on the retrieved information.

We have designed the first form of this tool in a way that the user can open several windows for each scenario. It means that, at the first stage of visualization tool, the user can select the scenario name, and then the second stage which contains the related scenario will open. This enables users to go back to the first form and select another scenario, while the first selected scenario is still open, and thereby draw a comparison between the charts of two or more scenarios.

For illustrating the information on each link, we retrieve different information (cost, $\mathrm{CO}_{2}$, and load) related to the links from the database. Then, we send this data to the Graph Listener to present them in the graph. This Listener will activate whenever the user pushes the related button of each data to show related information on each link. It can support interactivity of the visualization module. A sample screenshot of the link-based view is shown in Figure 3. The figure illustrates the amount of $\mathrm{CO}_{2}$ emission related to each link in the transport network. The data which is presented in the following diagrams is coming from the EastWest Transport Corridor $\mathrm{II}^{2}$ project (Holmgren et al, 2012b).

For the purpose of illustrating the route-related information in the route-based view, more complex queries have been designed. Furthermore, a chart is drawn to compare the information for all routes of a scenario with each other.

One screenshot of the route-based view is shown in Figure 4. The charts are drawn in the route-based view, because the charts are supposed to compare the information for all routes of a scenario to each other.

\footnotetext{
${ }^{2}$ http://www.ewtc2.eu/
}

$13^{\text {th }}$ WCTR, July 15-18, 2013 - Rio de Janeiro, Brazil 


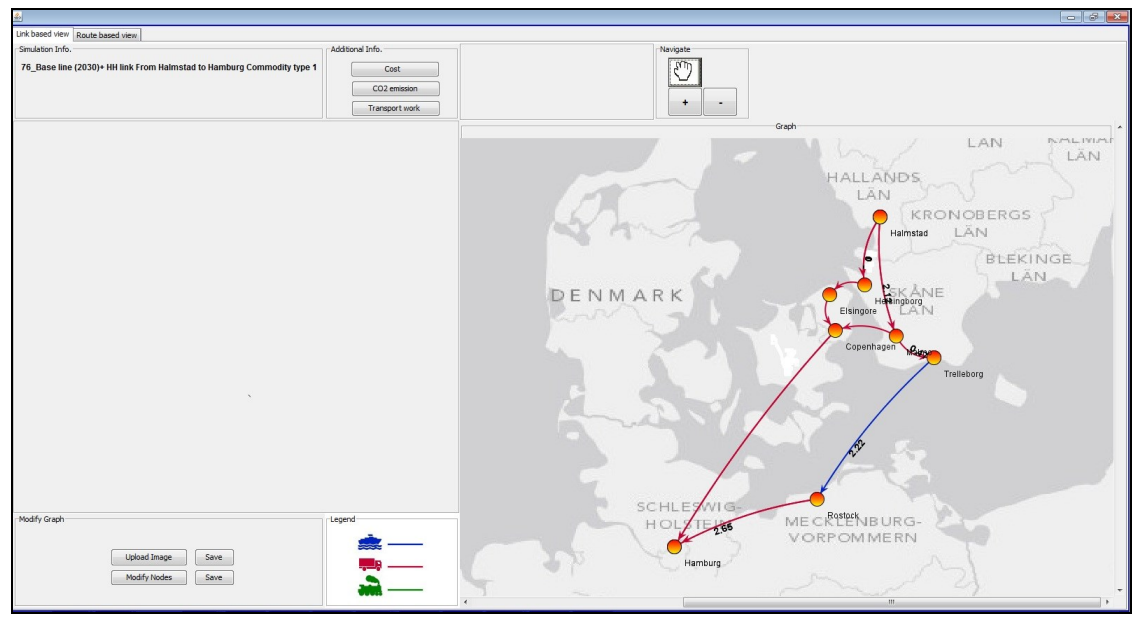

Figure 3 - Sample screenshot of the link-based view for a specific scenario

We also enhance the module with an ability which enables user to select an image and upload it as background for a specific scenario. Then for the next run of the visualization tool, the tool shows the right background which is selected and uploaded by the user for each scenario. The benefit of this feature is that in case of existence of two nodes which are too far from each other, the user can customize the map and cut the empty gaps between the nodes and upload it as a new background for the current scenario. This tool also has the capability to move the nodes and change their location on the map. In this case, the user can upload a map and check if the positions of nodes on the map are suitable or not. Then, he/she can adjust the nodes' places by moving nodes until it reaches a desirable position.

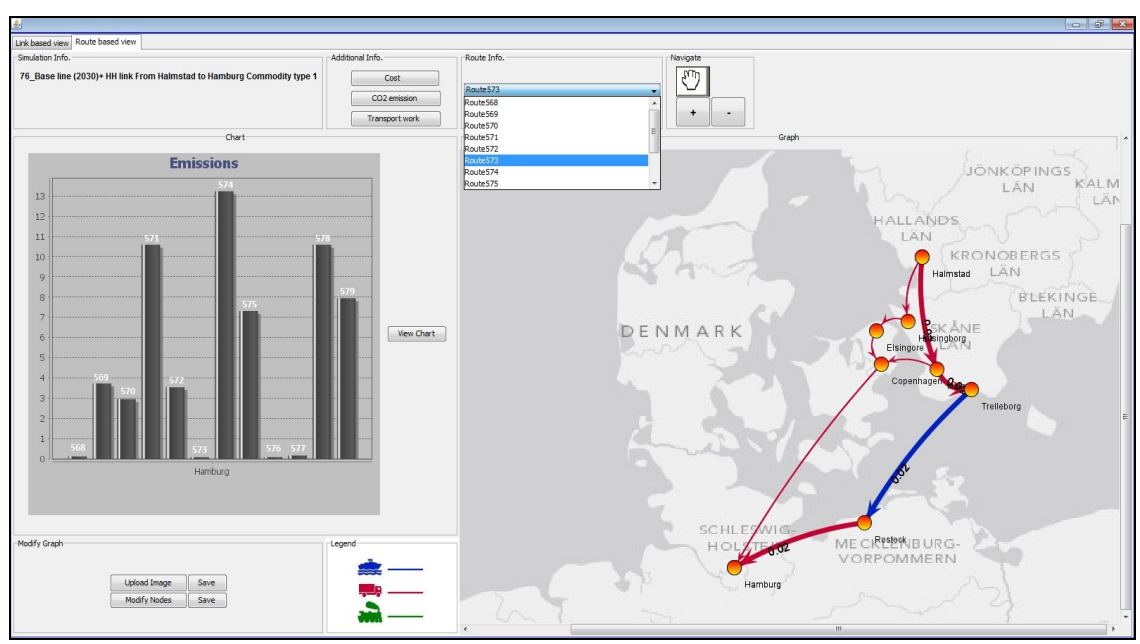

Figure 4 - A screenshot on the interactive tool for visualization of transportation simulation data, showing routebased view of a specific scenario

$13^{\text {th }}$ WCTR, July 15-18, 2013 - Rio de Janeiro, Brazil 


\section{Visualization of data from transportation simulation systems HAJINASAB, Banafsheh; DAVIDSSON, Paul; LÖWGREN, Jonas; PERSSON, Jan. A}

Based on the feedback from the users, we also designed a set of complementary diagrams, illustrating the aggregated results of several simulation runs of each simulation scenario. In this example simulation study, we have 5 different simulation scenarios and for each scenario there are more than 25 simulation runs. In the below diagrams (Figures 5a, 5b), the aggregated simulation results for two sample scenario are illustrated. The diagrams show how transport work distribution over the transport corridor will change in different simulation scenarios. Figure 5a illustrates the current situation (2010) scenario, while Figure $5 \mathrm{~b}$ is showing the Base line (2030) scenario where a distance-based road charging of 0.15 Euro/km for heavy trucks in the EWTC region is introduced (Holmgren et al, 2012b). The user can understand from the diagrams that by applying the specified distance-based road charging in 2030, how the amount of transport work will change, e.g. the load on the truck link between Kaunas-Klaipeda will considerably increase or the load on ferry link between Trelleborg and Rostock will slightly increase.
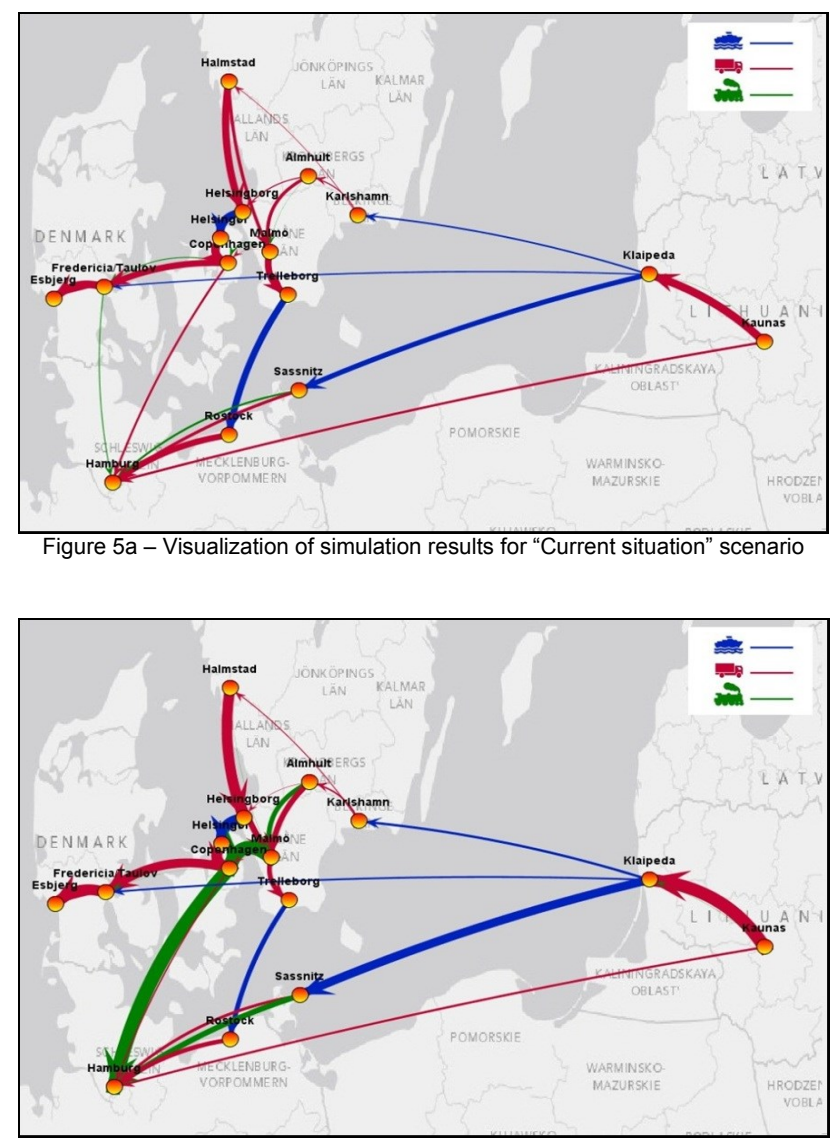

Figure $5 b$ - Visualization of simulation results for Base line (2030) scenario with a distance-based road charging $13^{\text {th }}$ WCTR, July 15-18, 2013 - Rio de Janeiro, Brazil 
Visualization of data from transportation simulation systems

HAJINASAB, Banafsheh; DAVIDSSON, Paul; LÖWGREN, Jonas; PERSSON, Jan. A

\section{EVALUATION AND USER FEEDBACK}

To investigate how the system can help decision makers to use simulation results more effectively, we did an evaluation by presenting the system to the users and asking for feedback through a questionnaire. According to the results of the questionnaires, the users found the diagrams showing aggregated results related to all simulation scenarios very useful. They are also more interested to see the simulation results for each route rather than links; even though they expressed that link-based data can also be useful in some special cases. All the respondents think that a visual representation of simulation data is definitely more useful than the current non-interactive and paper based solutions (e.g. tables and Excel sheets) for communicating the simulation results. Furthermore, the participants were mostly satisfied by the organization of the information and the interface of the visualization toolkit. They have also some suggestions for improving the visualization toolkit e.g. the use of colours in the bar charts and the possibility to make the scenario of their own by using the visualization toolkit.

Based on the positive feedbacks received during presentation of the module for users and from the questionnaire, we believe that the results from this study and the preliminary version of the visualization module can lead to future developments of visualizing the transportation simulation data for related stakeholders.

To increase the validity of research we have selected different methods to collect data and observe facts. Several interviews with users, experts, and the TAPAS development team are also conducted. In addition, the published documents of TAPAS; including the real data of database are reviewed and triangulation is used in this study's quest to discover relevant facts.

\section{CONCLUSION}

Visualization techniques can be used to increase the readability and usability of transportation simulation systems. To investigate how visualization can be applied to transportation context, we studied TAPAS as a real case and tried to specify user requirements related to visualization, design appropriate functionalities, and develop a visualization tool for TAPAS.

As an overall conclusion and in the light of our findings from the TAPAS case; firstly, visualization related requirements of users are identified and analysed for each group of users. Then the visualization capabilities are applied to each requirement. In the next step, the main features and functions to visualize outputs of system are designed. Finally, visualization tool is developed and is evaluated by system users to ensure that the developed tool can satisfy user's requirements.

In future, we plan to further evaluate functionality and usability of the tool to find out if the visualization tool can contribute to readability and usability of results.

A possible future improvement of the visualization tool can lie in investigating methods and techniques for visualizing input of TAPAS in order to be able to design the simulation scenarios using the visualization tool.

$13^{\text {th }}$ WCTR, July 15-18, 2013 - Rio de Janeiro, Brazil 
Visualization of data from transportation simulation systems HAJINASAB, Banafsheh; DAVIDSSON, Paul; LÖWGREN, Jonas; PERSSON, Jan. A

Furthermore, we can complement this user study further by investigating different decisionmaking methods in transportation field to understand how decisions in the transportation context are usually made. This has the potential to help design more effective and usable visualization models.

\section{ACKNOWLEDGEMENTS}

This research has been funded by the Swedish Transport Administration. We would like to thank all the participants in the interviews, and Johan Holmgren for their support during this research.

\section{REFERENCES}

Aris, A. (2008). Visualizing \& Exploring Networks Using Semantic Substrates., PhD thesis, Department of Computer Science. College Park, University of Maryland.

Becker, R. A., Eick, S. G. and Wills, G. J. (1995). Visualizing Network Data, IEEE Transactions on Visualizations and Graphics, Vol. 1, No. 1, pp. 16-28.

Bederson, B., Shneiderman, B. (2003). The Craft of Information Visualization: Readings and Reflections, Morgan Kaufmann.

Bergkvist, M., Davidsson, P., Persson J. A. and Ramstedt, L. (2005). A Hybrid MicroSimulator for Determining the Effects of Governmental Control Policies on Transport Chains, in: Lecture Notes in Computer Science: Vol. 3415. Multi-Agent and MultiAgent-Based Simulation, Springer, 236-247.

Boehm, B. (1988). A Spiral Model of Software Development and Enhancement, IEEE Computer, vol. 21, no. 5, May, 1988, pp .61-72.

Brasseur, L. E. (2006). Visualizing technical information: a cultural critique. Baywood Pub, Amityville, N.Y.

Breitkreut, B. J, Stark, C. and Tyers, M. (2003). Osprey: a network visualization system. Genome Biology, Vol 4, Issue 3, R22.

Brunner, D., Cross, G., McGhee, C., Levis, J. and Whitney, D. (1998). Toward increased use of simulation in transportation. In proceedings of 1998 Winter Simulation Conference, USA, pp. 1169-1175.

Card, S. K., Mackinlay, J. D. and Shneiderman, B. (1999). Readings in Information Visualization, Morgan Kaufmann Publishers.

$13^{\text {th }}$ WCTR, July 15-18, $2013-$ Rio de Janeiro, Brazil 
Visualization of data from transportation simulation systems HAJINASAB, Banafsheh; DAVIDSSON, Paul; LÖWGREN, Jonas; PERSSON, Jan. A

Chen, C. (2004). Information Visualization: Beyond the Horizon, 2nd edition, Springer-Verlag New York Incorporated.

Davidsson, P., Holmgren, J., Persson, J. A. and Ramstedt, L. (2008). Multi agent based simulation of transport chains. In Proceedings of the 7th International Joint Conference on Autonomous Agents and Multiagent Systems (AAMAS'08), Estoril, Portugal, pp. 1153-1160.

De Jong, G., Ben-Akiva, M. (2007). A micro-simulation model of shipment size and transport chain choice. Transportation Research Part B: Methodological, Vol. 41, Issue 9, pages 950-965.

Di Battista, G., Eades, P., Tamassia, R., and Tollis, I. G. (1998). Graph Drawing: Algorithms for the Visualization of Graphs. Upper Saddle River, N. J: Prentice Hall.

Eades, P. (1984) A heuristic for graph drawing., Congressus Numerantium, Vol. 42, pp. 149160.

Fishburn, P. T., Golkar, J. and Taafe, K. M. (1995). Simulation of transportation systems, In proceedings of 1995 Winter Simulation Conference, pp. 51-54.

Hajinasab, B. (2011). Visualization of data from transportation simulation systems. Master thesis, Department of Computer Science, Malmö University.

Hajinasab, B., Davidsson, P., Löwgren, J., \& Persson, J. A. (2012). A visualization toolkit for transportation simulation systems. In proceedings of the 7th Nordic Conference on Human-Computer Interaction: Making Sense through Design, pp. 793-794.

Herman, I., Melancon, G. and Marshall, M. S. (2000). Graph visualization and navigation in information visualization: a survey. IEEE Transactions on Visualization and Computer Graphics, Vol 6, No 1, pp. 24-43.

Hixson, C. L. (2006). Visualization for Project Development, NCHRP Synthesis 361. National Cooperative Highway Research Program. Transportation Research Board, Washington,D.C.

Hoen, P. J., Redekar, G., Robu, V. and La Poutre, H. (2004). Simulation and visualization of a market-based model for logistics management in transportation. In proceedings of the Third International Joint Conference on Autonomous Agents and Multiagent Systems (AAMAS'04), pp. 1218-1219.

Holmgren, J., Davidsson, P., Persson, J. A. and Ramstedt, L. (2007). An Agent Based Simulator for Production and Transportation of Products, In proceedings of 11th World Conference on Transport Research, Berkeley, USA, pp. 8-12.

$13^{\text {th }}$ WCTR, July 15-18, $2013-$ Rio de Janeiro, Brazil 
Visualization of data from transportation simulation systems HAJINASAB, Banafsheh; DAVIDSSON, Paul; LÖWGREN, Jonas; PERSSON, Jan. A

Holmgren, J., Davidsson, P., Ramstedt, L. and Persson, J. A. (2012a). TAPAS: A MultiAgent-Based Model for Simulation of Transport Chains. Simulation Modelling Practice and Theory, Elsevier, Vol 23, pp. 1-18.

Holmgren, J., Dahl, M., Hajinasab, B., Davidsson, P. and Persson, J. A. (2012b). The EastWest Transport Corridor Region: A micro-level simulation study. Corridor Capacity Support (Work Package 6), EastWest Transport Corridor II.

Hughes, R.G. (2008). Toward an Expanded Research Agenda for Visualization in Transportation: Incorporating SAFETEA-LU Directives for 'Planning'. Institute for Transportation Research and Education, North Carolina State University, Raleigh, NC.

Hughes, R.G. (2010). Freight data visualization: A "Pivotal" point in the development of visualization applications in transportation. In proceedings of the 2011 Transportation Research Forum, Long Beach, CA.

Kaufmann, M., Wagner, D. (2001). Drawing Graphs: Methods and Models, volume 2025 of Lecture Notes in Computer Science. Springer.

Mazza, R. (2009). Introduction to Information Visualization. Springer, London.

Post, G. V., Anderson, D. L. (2002). Management information systems: Solving business problems with information technology. New York: McGraw-Hill Irwin.

Ramstedt, L., Davidsson, P., Holmgren, J. and Persson, J.A. (2007). On the Use of Microlevel Simulation for Estimation of the Effects of Governmental Control Policies, 11th World Conference on Transport Research, Berkeley, USA.

Richter, R., et. al. (2003). Visualization in Transportation: A Guide for Transportation Agencies, AASHTO Task Force on Environmental Design, American Association of State Highway and Transportation Official (AASHTO).

Shneiderman, B., Aris, A. (2006). Network visualization by semantic substrates. IEEE Transactions on Visualization and Computer Graphics, Vol 12, No 5, pp. 733-740.

Ware, C. (2012). Information Visualization: Perception for Design, Morgen Kaufmann, USA. Wymore, W. (1993). Model-Based Systems Engineering, CRC Press, Boca Raton. 



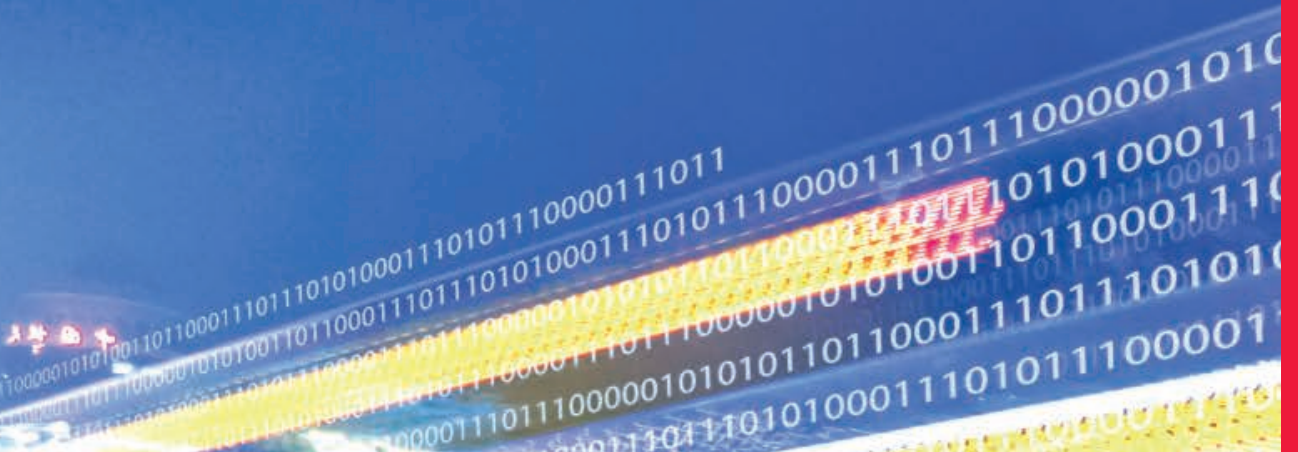

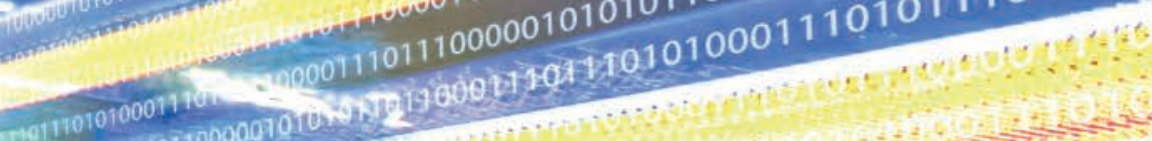

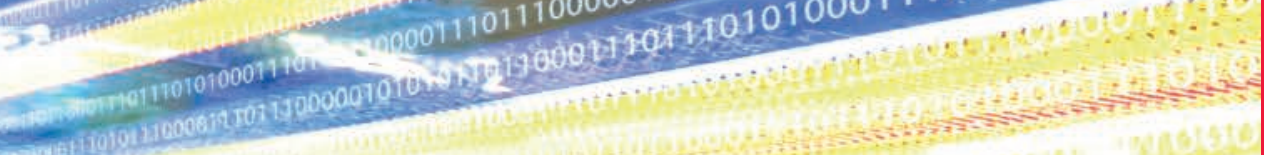

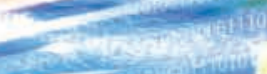

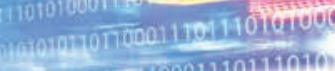

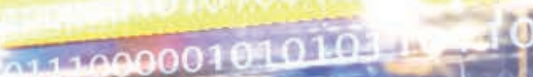

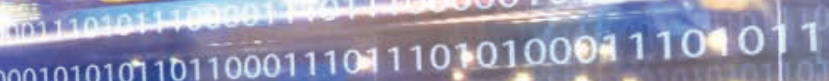
a t Not thot

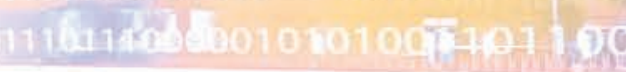

